\title{
The Shape of Games to Come: Critical Digital Storytelling in the Era of Communicative Capitalism
}

by

Sarah E. Thorne

A thesis submitted to the Faculty of Graduate and Postdoctoral Affairs in partial fulfillment of the requirements for the degree of

Doctor of Philosophy

in

Cultural Mediations

Carleton University

Ottawa, Ontario

(C) 2018, Sarah E. Thorne 


\section{Abstract}

The past decade has seen an increase in the availability of user-friendly game development software, the result of which has been the emergence of a genre of reflexive and experimental games. Pippin Barr, La Molleindustria's Paolo Pedercini, and Davey Wreden are exemplary in their thoughtful engagement with an ever-expanding list of subjects, including analyses and critiques of game development, popular culture, and capitalism. These works demonstrate the power of games as a site for critical media theory. This potential, however, is hindered by the player-centric trends in the game industry that limit the creative freedom of developers whose work is their livelihood.

In the era of communicative capitalism, Jodi Dean argues that the commodification of communication has suspended narrative in favour of the circulation of fragmented and digestible opinions, which not only facilitates the distribution and consumption of communication, but also safeguards communicative capitalism against critique. Ultimately, the very same impulse that drives communicative capitalism is responsible for the player-centric trends that some developers view as an obstacle to their art. Critical game studies has traditionally fallen into two categories: those that emphasize the player as the locus of critique, such as McKenzie Wark's trifler or Mary Flanagan's critical play, and those that emphasize design, as in Alexander Galloway's countergaming, Ian Bogost's procedural rhetoric, and Gonzalo Frasca's theory of simulation. Yet, as a result of the decline in symbolic efficiency that is characteristic of communicative capitalism, these approaches are limited in their ability to effectively respond to the current social and economic situation. 
Dean's conditions for critical media theory, however, provide a framework for theorizing how the complex interaction between a game's narrative and mechanics might be adapted to resist and disrupt communicative capitalism. Drawing on Freudo-Lacanian psychoanalysis, this project analyzes a selection of narrative games through the clinical structures of neurosis, perversion, and psychosis to understand how players interact with these systems. This perspective provides the basis for examining the subject of communicative capitalism and imagining the shape of critical games to come. 


\section{Acknowledgements}

This project would likely have taken a very different direction were it not for growing up in a household with a gaming console; for that, a special thank you is owed to my parents, and also to my brother and sister, who taught me determination by showing no mercy when we gamed.

I am indebted to Dr. Brian Greenspan, whom I owe thanks not only for his support and supervision throughout this project, but also for opening up opportunities that I would never have imagined. Being a part of the Hyperlab has given me the chance to explore the practical side of theory and to collaborate on exciting digital projects. These experiences will be invaluable to future endeavours.

I would like to thank Dr. Aubrey Anable and Dr. Chris Russill for joining my committee. Your feedback on this project has been insightful and very much appreciated. I would also like to thank Dr. Stephanie Boluk and Dr. Stuart Murray for taking the time to participate in the defense. I am also grateful to Trevor Smith, who has been a constant source of support and encouragement, and also an excellent sounding board. And, though there are too many of you to name, thank you to my friends and colleagues.

Finally, I would like to acknowledge that this research would not have been possible without the generous funding provided through the Joseph-Armand Bombardier Canada Graduate Scholarship and the J. James Mackie Endowment for Graduate Scholarships in Human-Technology Interaction. 


\section{Table of Contents}

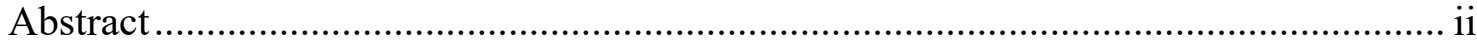

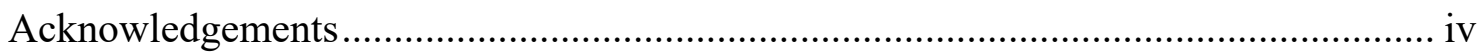

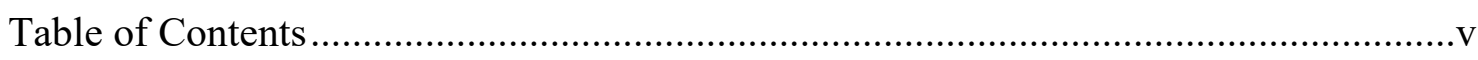

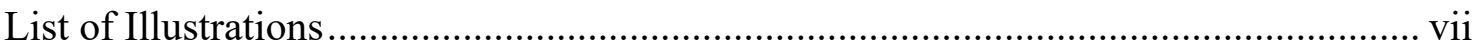

\section{Section I Narrative}

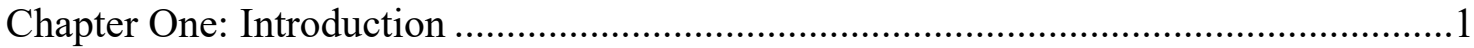

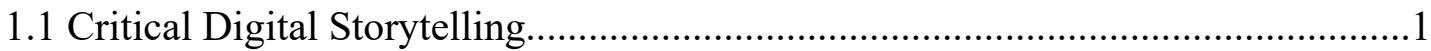

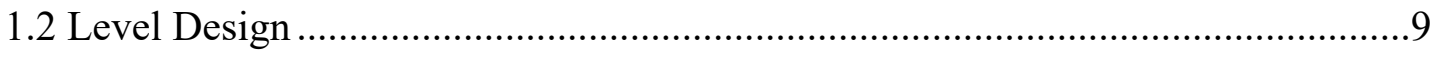

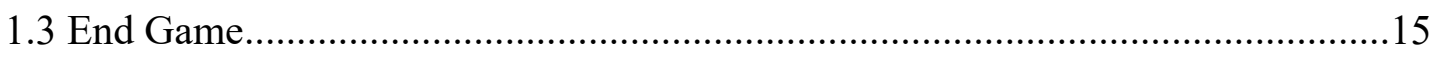

Chapter Two: The Digital Transformation of Storytelling ............................................17

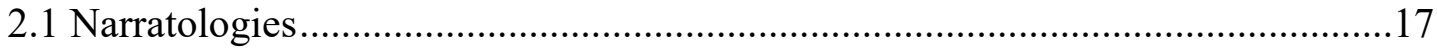

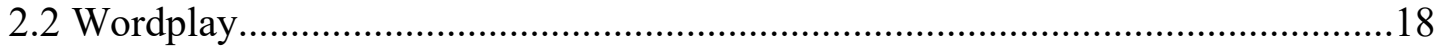

2.3 Ludology Vs. Narratology ...........................................................................24

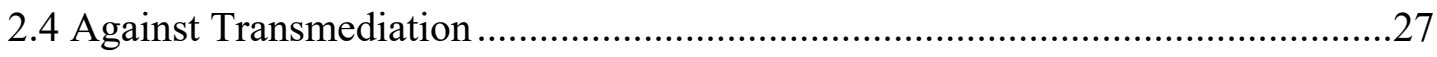

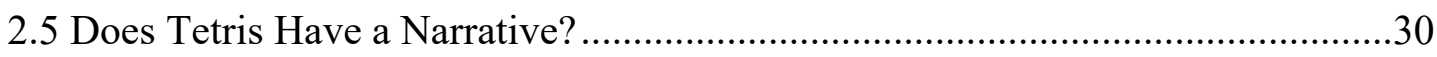

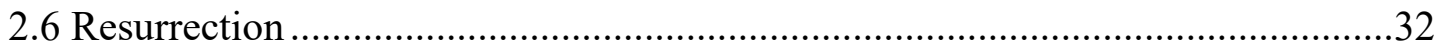

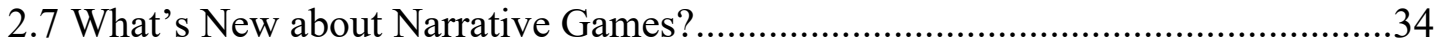

\section{Section II Agency}

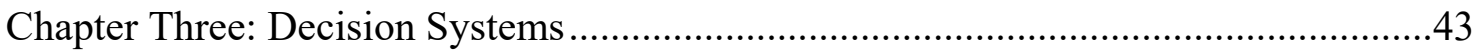

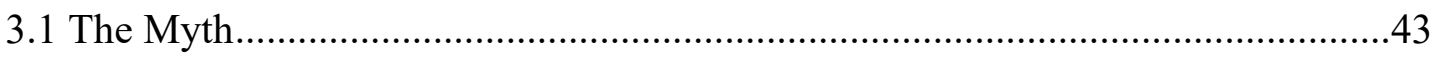

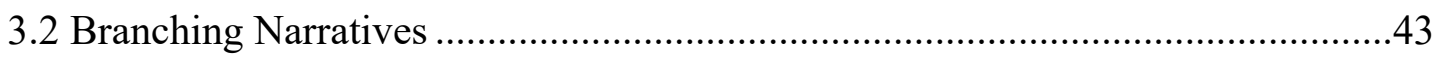

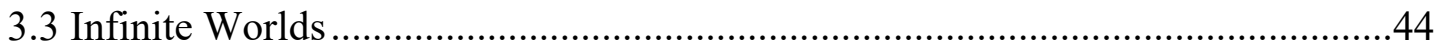

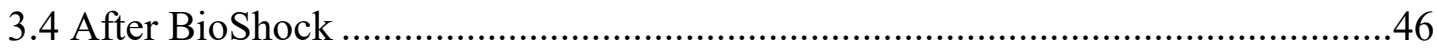

Chapter Four: New Approaches to Narrative Agency …………………………….....54

4.1 Algorithmic Approaches: The Novelist .................................................................57

4.2 Who Will You Become?: The Walking Dead ....................................................57

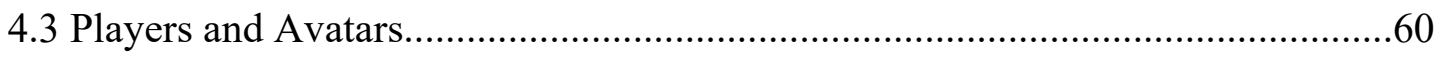

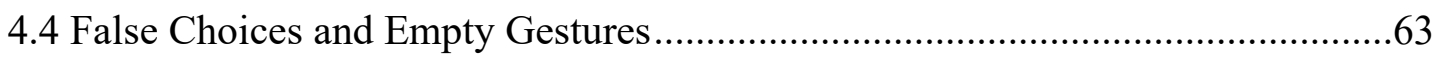

4.5 Agency and the Player-Avatar Relationship.........................................................66 


\section{Section III: Enjoyment}

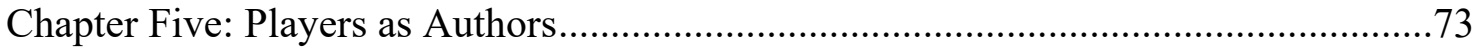

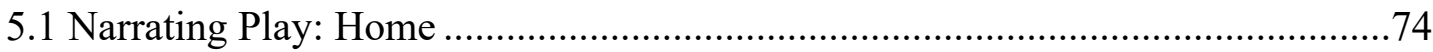

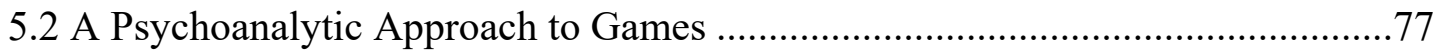

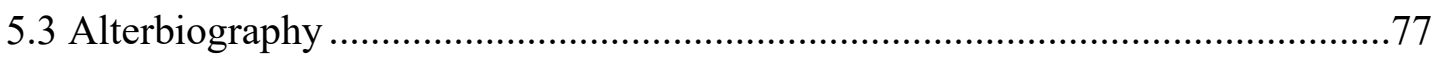

Chapter Six: Perverse and Interpassive Gaming

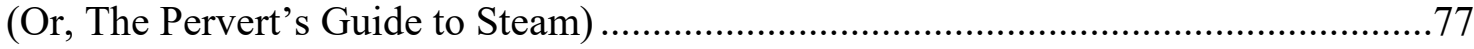

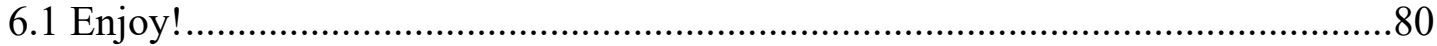

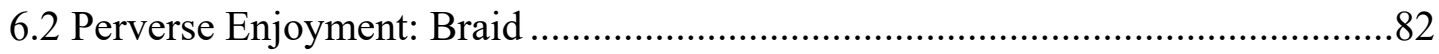

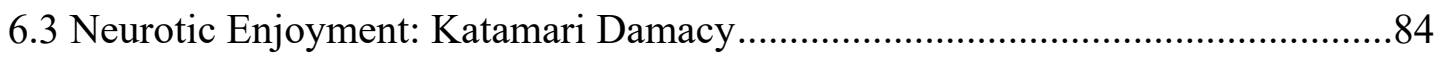

6.4 Psychosis and Ethics: Violent Games ....................................................... 92

\section{Section IV: Critical Games}

Chapter Seven: Industry, Agency, and Critical Media Studies .................................97

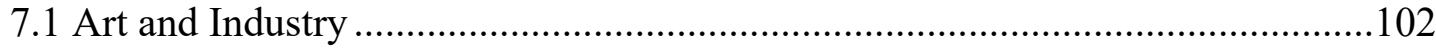

7.2 On Making Games: The Magic Circle ......................................................... 105

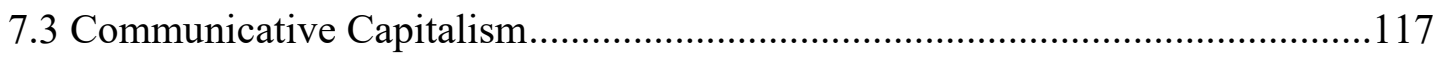

7.4 Agency and the Critical Role of Narrative: The Stanley Parable .......................117

7.5 The Beginner's Guide to Critical Media Theory ............................................. 117

Chapter Eight: The Shape of Games to Come .......................................................126

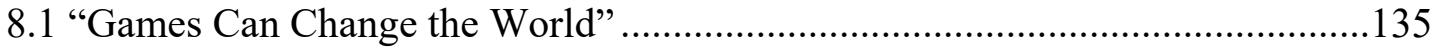

8.2 The Subject of Communicative Capitalism ............................................... 141

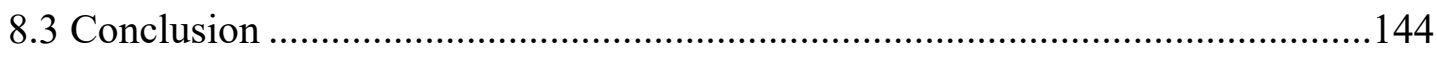

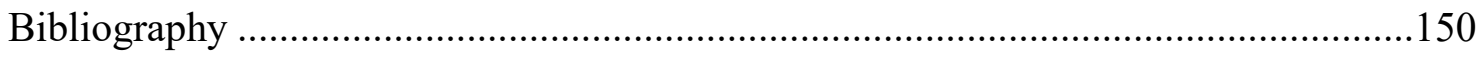




\section{List of Illustrations}

Fig. 2.1: an image by Ian Bogost that depicts Janet Murray and Espen Aarseth sparring to settle the Ludology vs. Narratology debate. "Videogames Are a Mess,"

Bogost.com, 3 Sept. 2009 ........................................................................... ii

Fig. 4.1: screenshot from episode 2 of Telltale's The Walking Dead (2012)................... iv

Fig. 4.2: screenshot from episode 2 of Telltale's The Walking Dead (2012).....................

Fig. 5.1: screenshots from the opening scene of Benjamin Rivers' Home (2012) .......... vii

Fig. 7.1: screenshot of the game from Davey Wreden's The Stanley Parable (2013).........1

Fig. 7.2: screenshot of Davey Wreden's The Beginner's Guide (2016)...........................

Fig. 7.3: screenshot from Davey Wreden's livestream on Twitch ................................. 1 


\section{Section I Narrative}

\section{Chapter One: Introduction}

\subsection{Critical Digital Storytelling}

Writing in 2006, Alexander Galloway anticipates the arrival of an independent gaming movement that realizes "its true potential as a political and cultural avant-garde" (126). These new games, he argues, will redefine play through "radical action," a term that he uses to describe practices of countergaming that, rather than attending to the aesthetics of a game, redefine mechanics and algorithms to critique gameplay (125). While Galloway states that, at the time of writing, "[a]n independent gaming movement has yet to flourish" (126), the past decade has seen an increase in the availability of accessible, user-friendly game development software, which has resulted in the creation of innumerable innovative and experimental games. Access to free game development software like Game Maker and Twine has enabled users with little or no programming experience to create and distribute their own interactive games. Unfettered by expectations, the independent production of games promises to be a space for exploring the potential of the medium free from the constraints of market trends and player demands.

Games such as Everything Unlimited Ltd.'s The Beginner's Guide (2015) and Question's The Magic Circle (2015) are exemplary as critical works that examine the process of making games and raise important questions about the relationship between developers and their audience in a medium that promises players agency. These games challenge player-centric trends in an industry that insists that for games to be successful, developers must hand over narrative control to players. While technical limitations do not 
yet allow players to fully author their own experiences, features like complex decision systems, branching narratives, and open worlds necessitate that developers must concede some control over narrative direction to allow players to shape the story and its conclusion. These trends are at odds not only with the creativity of developers, but also the countergaming project identified by Galloway, which did not anticipate the significant pressure that industry conventions would exert on independent (indie) developers.

The desire for a truly dynamic and interactive narrative has been an aspiration of storytelling long before its popularization in games; it is a theme that was previously explored in works like Jorge Luis Borges' short story "The Garden of Forking Paths" (1941), "Choose Your Own Adventure" novels from the 1980s, and later hypertext fiction. Examining ergodic literature, Espen Aarseth (1997) states that, Cybertext ... is not a "new," "revolutionary" form of text, with capabilities only made possible through the invention of the digital computer. Neither is it a radical break with old-fashioned textuality, although it would be easy to make it appear so. Cybertext is a perspective on all forms of textuality, a way to expand the scope of literary studies to include phenomena that today are perceived as outside of, or marginalized by, the field of literature-or even in opposition to it, for ... purely extraneous reasons. (18)

Experimental literature, in all of its analog and digital forms, explores the potential and limits of narrative agency, and games are only recently joining this conversation. Current trends promoted in advertisements often describe games as a medium for multilinear storytelling that allows players to make meaningful choices that direct narrative 
outcomes. Irrational Game's BioShock series and Telltale's The Walking Dead, for instance, promise players compelling interactive narratives that offer players choices to create more personalized experiences. While BioShock has critiqued the possibility of creating truly player-driven narratives, others continue to explore this potential through the more technical features of games, such as algorithms and procedurally-generated content.

The question of how digital technology has transformed storytelling is not particularly new, as the immersive, interactive, multimedia, and multilinear narrative experiences offered by new digital technologies have been thoroughly examined by theorists such as Marie-Laure Ryan, Espen Aarseth, Janet Murray, and George Landow, to name only a few. This question, however, finds renewed significance in the context of game development and the strategies adopted by developers to create increasingly complex player-driven narratives. Though the field of game studies has often been divided between ludological and narratological approaches to games, this project seeks to reconcile this division to move beyond this well-worn debate to examine the critical potential of games. While this project must contend with the varied perspectives offered in this debate, the aim is not to provide a comprehensive account of the new narrative possibilities offered by games, but rather to question the implications of handing narrative control over to players. At its core, this project asks: if given the freedom, what kinds of stories will players tell themselves, and, perhaps more importantly, what kinds will they avoid? Ultimately, the question of whether such control is real is secondary to players' perceptions of their ability to manipulate the game world, as the concerns that this project raises focus on player experience, rather than philosophical questions about 
the existence of freedom and control. Adopting a psychoanalytic approach, this project analyzes agency at the level of fantasy to explore the political implications of this trend. This perspective enables a dynamic analysis of the cognitive and social value of narrative that is also able to move beyond concerns about the technical challenges associated with facilitating player agency to address more fundamentally how players engage with these game systems and the limits that one already imposes on oneself.

Examining the metanarratives of AAA and indie titles reveals a complex debate about the limits of agency in games and the cultural importance of games as a narrative medium. Popular titles like BioShock and The Walking Dead provide an insight into the limits of narrative and agency from the perspective of big-budget games beholden to publishers, franchise owners, and share-holders. In contrast, Orthogonal's The Novelist (2013) reveals the challenges of the passionate and risky projects of indie developers who work on complex narrative systems for smaller scale games. Each perspective contributes to the broader picture of how developers respond to issues of agency and authorship, and how player-centric trends in the industry threaten the future of games as a critical medium. As the game industry pushes to satisfy consumer demands for increased agency, it is imperative to consider the consequences of giving players the ability to direct narrative content. Narrative serves as both entertainment and a way to transmit culture and allegories about humanity. Yet, if games follow industry trends that give players the ability to direct a narrative, the allegorical significance of the work is diminished. Take for instance a work like Upper One Games' Never Alone (Kisima Ingitchuna) (2014), which recounts a traditional story of the Iñupiat people of Alaska about community, interdependence, and hardship. The game was created in consultation with the 
community's storytellers and elders, and was designed to accurately represent their culture and values. In the game, players enact the events of a story about Nuna, a young girl, and her fox, who seek the source of the blizzards that have brought hardship to her home. By navigating the challenging landscape of the arctic north as these characters in tandem, players experience the game's allegory about interdependence firsthand, rather than indirectly as an oral story. The strength of the game's story comes from its careful crafting of the player's experience. Had the game's developers allowed players to intervene in the story, the game would not hold the same allegorical significance. While Never Alone is not representative of the kinds of critical works examined herein, its importance to the Iñupiat signals the value of storytellers and storytelling in games.

Narrative plays a central role in our lives and for this reason this project incorporates a psychoanalytic approach to emphasize not only how "we attempt to obtain a narrative understanding of ourselves" (Ricœur 33), but also the importance of confronting difficult stories that one might be inclined to avoid. In this process of narrativization, as Paul Ricœur notes in his discussion of the role of narrative in psychoanalysis, there is a gap between the events that we live and our telling of those events; as he writes, "we learn to become the narrator and the hero of our own story, without actually becoming the author of our own life" (32). Building on this insight, this project examines the value of confronting difficult narratives and theorizes the shape that such narratives should take. Drawing on Jacques Lacan's treatment of the psychical structures of neurosis and psychosis, this project argues that the structure of a narrative is essential to its success as a critical work. As Dean argues, communicative capitalism relies on the decline of symbolic efficiency, which is also characteristic of psychosis. A 
psychoanalytic methodology enables this project to theorize the critical role of storytelling in the era of communicative capitalism by examining how narrative structures might disrupt the subject's capture in the circuits of drive.

Current trends in games reveal a desire to eliminate the gap between author and hero by creating a world where we author our own experiences, which ultimately creates a fantasy space removed from reality. While open worlds and customization might create enjoyable game experiences, developers see this trend as a shift in their position from authors of virtual worlds to creators of game assets that set the stage for players. As a result, not only is their creative control sacrificed, but also their ability to create works that intellectually challenge players. As an example, one might consider the impact of this trend on the representation of minorities in games. As Adrienne Shaw argues, giving players the ability to create and customize their own avatars does nothing to challenge players with narrow views of the world (143). Although diversity is not the central concern of this project (and the games examined are largely unremarkable as examples of diversity), the strategies that these games employ to facilitate critical reflection are beneficial for addressing diversity, in part, because the experiences that they offer are not optional. In contrast to games with customizable avatars, those that feature minorities as three-dimensional playable characters force players to occupy their worldview, which, as Luca Papale argues, can facilitate understanding and sympathy (8).

It is important to note that my approach to game studies, the game industry, and player involvement is in conflict with work by theorists like Henry Jenkins and Jane McGonigal, who argue for the benefits of participatory culture. Such benefits, however, come at a cost. As Jordan Thomas notes in his talk about his experience making The 
Magic Circle at the 2016 Game Developers Conference (GDC), it is precisely this drive for consumer inclusion that has pressured indie developers to return to the player-centric and market-driven values of the AAA game companies that they had left. Instead of independent games serving as a space to freely explore one's creativity, indie developers have found themselves in a position where they must yield to consumer demands or risk their financial future. Contrary to those in favour of participatory culture, this project aligns with Jodi Dean and other Marxist thinkers who are critical of capitalism to explore the revolutionary potential of narrative by embracing the strategies that they adopt to resist capitalism's influence over media production.

Theorists like Gonzalo Frasca, Mary Flanagan, and McKenzie Wark have also addressed the potential for games as a critical medium; as will be developed, however, these approaches are now a bit dated to address the current political and economic environment, which has seen a transformation over the last decade as a result of the 2008 economic crisis and far rightwing movements like GamerGate. These events have had a significant impact on the production and consumption of games, and although these texts remain important, more work needs to be done to reevaluate games as a critical medium. Gonzalo Frasca, in his Master's thesis titled Videogames of the Oppressed (2001), discusses the potential for games as simulations to represent real situations to "[foster] critical thinking and discussion" (ix). In Critical Play (2009), Mary Flanagan theorizes play as a site of subversion and critique. Flanagan draws attention to individual artists who experiment with games to subvert traditional play mechanics and the so-called “serious" games that highlight social causes. In Gamer Theory (2007), McKenzie Wark extends gameplay to examine the physical world as a gamespace to theorize a similar 
kind of critical play through the "trifler," a player who subverts the aims of the game and explores the limits of its rules and mechanics (endnote 034). In Gaming (2006), Alexander Galloway instead envisions an approach to game design that follows the traditions of counter-cinema; countergaming, he argues, subverts mainstream conventions and aesthetics to create transgressive games. While critical forms of play are an essential part of critiquing commercial games, Galloway's emphasis on the developer as the locus of critical engagement will prove particularly relevant to challenging playercentrism in the era of what Jodi Dean refers to as communicative capitalism, where symbolic efficiency and narrative have been suspended (Blog Theory 31). Drawing on Dean's work, my aim is to reconsider the necessary conditions for games to function as a kind of critical media theory.

Although this project is indebted to indie games, it is not fundamentally an account of this movement. It is not a theory of play, like that found in Flanagan's Critical Play (2009), nor an account of contemporary “gamespace” from Wark's Gamer Theory (2007). Instead, this project occupies a middle ground between developer and player, between production and consumption, to examine the systems that underpin our experiences of play to theorize how design can disrupt player expectations to fascinate critical thinking. While the emphasis is on the construction of critical games, rather than player-directed experiences, it is also not an instructive theory of design. While these moments can be intentionally designed, as will be discussed, there is no guarantee that these efforts will succeed in affecting players as planned. First and foremost, this project is an account of narrative, agency, and critical media theory; and as such, this work is 
intended to appeal to a diverse audience, despite its emphasis on games. This work holds relevance for the fields of narratology, critical theory, psychoanalysis, and game studies.

The games discussed herein were chosen for their contribution to discussions of agency and narrative (e.g. BioShock, The Walking Dead, and The Novelist), and for their reflection on games and game making (The Magic Circle, The Stanley Parable, and The Beginner's Guide). Although Pippin Barr and La Molleindustria's Paolo Pedercini offer excellent examples of critical games, their work has been omitted because it is not subject to the same financial concerns associated with industry and audience pressures. Creating experimental and critical games that go against mainstream trends requires a certain amount of risk, and indie game developers may not be willing to gamble with their financial future.

The following sections dedicated to narrative, agency, and enjoyment will serve to develop a framework for thinking through the player's interaction with and experience of narrative games. This work will culminate in the fourth section in an application of this psychoanalytic approach to games to both address the challenges of designing critical works and to propose two models for the shape of games to come that will challenge communicative capitalism.

\subsection{Level Design}

Chapter Two provides an overview of the development of a digital narratology designed to account for hypertext and other forms of digital storytelling. The aim of this section is to provide a working definition of narrative that will be necessary for contending with subsequent approaches to new media that dismiss digital narratives on 
the basis of their novelty and often fragmented transmission in communicative capitalism. Digital narratology provides a framework for describing and analyzing the narrative structures of nonlinear and multilinear texts. This section also contends with the disputed definitions of narrative often employed in game studies to move beyond those who subscribe to traditional definitions, such as that given in Aristotle's Poetics, which has largely dominated the field of narratology. The proposed alternative definition borrows from Rick Altman's A Theory of Narrative, as his work permits a broad and inclusive definition that may include not only interactive fiction, but also games.

Although digital narratology may have adapted to include games in its definition of narrative (Ryan 2006), the field of game studies has had its own contentious relationship with narratology. In the so-called "Ludology vs Narratology" debate, game theorists such as Jesper Juul (2001) and Gonzalo Frasca (2003) have challenged narrative approaches to games and have argued that the two are incompatible. Although Janet Murray supposedly put this debate to rest in 2005, it has continued to persist, as most recently seen in the work of Ian Bogost (2017). This chapter will address and critique relevant aspects of this debate and its influence on the field. As has been thoroughly examined throughout this debate in games studies, neither the ludological nor the narratological perspective is wholly adequate to provide a comprehensive analysis of the video game as a cultural object. Both the formal properties of the game and its narrative must be understood as interacting elements within a system, and not as mutually exclusive components of the user's experience. This debate remains of interest, however, as it provides insight into players' resistances to particular genres of narrative games that 
are seen as "less interactive" and are dismissed as a kind of interactive fiction and as therefore "not games."

Chapter 3 explores narrative decision systems and player agency through the popular BioShock series. Well-known for its critiques of agency and free will, the BioShock series addresses the nature of multilinear narratives, and ultimately critiques its own promise to offer a world where the player's decisions matter. Instead of the alternate endings that players had come to expect from the series, the conclusion of BioShock Infinite, the third iteration of the series, presents players with a world in which all narrative outcomes are possible and simultaneous, but are inaccessible to the player, as each exists as its own, separate dimension. What players get at the end of Infinite shares much in common with what Marie-Laure Ryan describes as the myth of the Aleph in her account of hypertext narrative. The term is a reference to the eponymous short story by Borges (1945), wherein the Aleph is described as a point in space that provides a window through which to see everything in the universe from all possible angles simultaneously. In "Beyond Myth and Metaphor," Ryan employs the story of the Aleph to mythologize the perspective that by randomizing the sequence of narrative events, hypertext can create a finite text that allows for an infinite number of stories. This account of narrative, however, provides a compelling model to consider in relation to the fascinating ending of BioShock Infinite, which offers a clever ruse that both explains away the player's inability to shape the outcome of the game, and illustrates the inherent technical difficulties of creating narrative worlds that give players an active role in their construction.

Chapter 4 examines the narrative decision systems of games after BioShock. In the post-BioShock era, a number of developers have taken BioShock's message as a 
challenge, and have attempted to design narrative structures that move beyond the unsatisfying form of branching stories by developing algorithms that generate content. This chapter takes Kent Hudson's The Novelist (2013) and Telltale Games's The Walking Dead (2012) as case studies for exploring new approaches that challenge the limited vision of narrative and player agency presented in the BioShock series. While Hudson's game relies on an algorithm to trace the player's interaction with the narrative, The Walking Dead shifts the narrative focus from outcomes to character interactions in order to avoid the challenges of content creation for multilinear games.

Chapter 5 responds to the approaches of the previous games that enable players to shape characters and relationships to consider more specifically how players engage with game narratives and the consequences of giving players control of the narrative through the example of Benjamin Rivers' Home (2012). In In-Game: From Immersion to Incorporation, Gordon Calleja explores how the player's construction of a narrative is always informed by both the scripted narrative and the player's own subjective interpretation of events and experiences in and out of the game. Home - a murdermystery game — takes advantage of the player's alterbiography by changing "subtly, almost imperceptibly" (Rivers) to reflect the player's interpretation of the events of the game, rather than providing players with choices that determine the direction of the game.

Chapter 6 elaborates on the player's experience of a game through Lacan's clinical structures of neurosis, perversion, and psychosis. Taking the game Braid (2008) as an exemplary work of proceduralism in practice (a game defined by its rules) and Katamari Damacy (2004) as its structural inverse (a game defined by free play and 
trifling), this chapter analyzes how players enjoy games as rule — and narrative — based activities. If games are, after all, meant to be played for fun, and if a game is foremost the execution of its rules, where is the enjoyment in playing, and how do subversive acts of play, such as trifling and cheating, fit into this project's concerns about narrative structures and player agency? For example, how can it possibly be fun to play as Sisyphus in Pippin Barr's collection of games called Let's Play: Ancient Greek Punishment (2012)? This myth is also the basis of Namco's successful Katamari games, where it puts players to work, requiring them to push around a ball to collect items in order to rectify a mistake made by the intractable King of all Cosmos. This structure of gameplay is the opposite of that found in Braid, wherein play is turned into work, as the player must execute specific procedures (rules) in order to solve puzzles. Developing Lacan's work on clinical structures, this section will argue that when play is turned into work (as demonstrated through Braid), the enjoyment of a game is structured like that of perversion $(\mathrm{a} \diamond \$)$, and when work is turned into play (Katamari), enjoyment is structured like neurosis $(\$ \triangleright a)$.

Chapter 7 examines Jodi Dean's theory of communicative capitalism and her conditions for critical media theory in a world dominated by fragmentation, opinions, and affects. In Blog Theory, Dean theorizes that the decline of symbolic efficiency has made meaning unstable. Ubiquitous digital media, she argues, do not produce narratives, but instead create a corpus of disconnected messages and posts that facilitate the commodification of communication. While Dean argues that the book is our best chance for producing critical media theory in our current media environment, I posit that games 
are also a strong contender and are congruent with Dean's outline for the necessary conditions for critical media theory, despite her critique of digitally produced works.

The possibility for games to act as critical media theory is best illustrated by Davey Wreden's The Beginner's Guide, an unusual game in that it invites players to analyze a collection of incomplete games with the explicit aim of not only uncovering the developer's intention, but also assessing his emotional state at the time of creating these works. In its conclusion, The Beginner's Guide turns this objective on its head, which forces players to reconsider how they approach not just this particular game, but all games. In so doing, The Beginner's Guide indirectly raises important questions about the implications of giving players control over narrative direction. The game succeeds as a kind of critical media theory not only because it incorporates techniques to disrupt player agency through its unplayable moments, but also because it directly confronts the problem of communicative capitalism and the circulation of affect and cultural capital. The Beginner's Guide offers a way to imagine other possible sites of critical media theory while staying true to the conditions that Dean outlines, and without lapsing into overly optimistic or uncritical views of technology and popular culture.

The final chapter concludes with a broader discussion of the obstacles faced by critical media theory in the current socio-political context. It examines the symptoms that have given rise to fascism not just in politics, but also in the gaming community. This far right movement has been given many names, but as G. M. Tamás (2000) writes, this rising form of what he calls "[p]ost-fascism finds its niche easily in the new world of global capitalism without upsetting the dominant political forms of electoral democracy and representative government." Similar trends in the gaming community have also 
served to support the goals of industry elites. In "How Video Games Are Fuelling the Rise of the Far Right" (2018), Alfie Bown argues that the connection between these movements and the gaming community is not the result of one co-opting the other, but rather that the very content and structure of many games supports right-wing ideologies, and presents them in an agreeable form. Although apt, this analysis lacks a rigorous consideration of how players interact with these game systems. By focusing on Lacan's

clinical structures of neurosis and psychosis, this chapter theorizes two possible responses to the decline of symbolic efficiency and the rise of post-fascism. These psychical structures describe the relationship between the subject and desire, or lack thereof, and provide a framework that illuminates the underlying symptoms of these movements. Similarly, the treatment of neurosis and psychosis provides a course of action for responding to these movements that corresponds to the analyst's approach to these conditions in clinical cases.

\subsection{End Game}

In sum, this project considers the impetus behind the creation of dynamic and adaptive narrative structures to consider not only how giving players control over narrative changes how stories are told, but also the cultural and political implications of this trend in game development. This analysis draws a parallel between Dean's critique of communicative capitalism and the obstacles that games face as an art form to theorize games as a possible site for critical media theory. Ultimately, the very same impulse that drives communicative capitalism is responsible for the player-centric trends in the game industry that developers view as an obstacle to their art. The Beginner's Guide provides 
an entry point into theorizing games as a form of critical media theory. The complex interaction between a game's narrative and mechanics provides a useful context for thinking through Dean's analysis of the book as a critical medium to explore how its tactics can be adopted by games to resist communicative capitalism and the rise of movements like Gamergate. 


\section{Chapter Two: The Digital Transformation of Storytelling}

\subsection{Narratologies}

The study of game narratives presents two obstacles: the first is classical narratology's resistance to the inclusion of digital works as a category of narrative, and the second is the objection to narratological approaches to the medium by game theorists. Both obstacles are the result of the Aristotelian definition of narrative that has pervaded much of narrative theory. As a field of study, narratology aims to formalize an approach to describing and categorizing the broad and diverse existence of narratives. As Roland Barthes notes, narratives are infinite, ubiquitous, and universal (79), and it is for this reason that the task of narratology is challenging. Theorists have approached narrative by employing a number of different strategies: Barthes, for instance, developed a descriptive approach modelled after Ferdinand de Saussure's semiotics for the reason that both narrative and language are unmanageable in size. Other theorists followed Aristotle and worked to delimit the field as much as possible by narrowing narratology to the study of print literature and emphasized features like story and discourse (e.g. the often taught Freytag pyramid). Theorists such as Seymour Chatman, Gérard Genette, and Gerald Prince instead defined narrative based on the presence on a narrator - a feature often absent in non-print media that audiences recognize as narrative (Ryan, Avatars 5). Poststructuralist approaches to narrative have since branched into a wide variety of areas that include feminist, ethical, cognitive, and digital approaches. Defining the boundaries of the field, however, continues to be a point of contestation among scholars. While some theorists opt for narrow definitions that restrict the term narrative to literary texts and 
exclude many media forms that most individuals would identify as narrative (e.g. drama, interactive fiction, games), others risk broad and inclusive definitions, which scholars like Marie-Laure Ryan fear will render the term narrative meaningless (7). This concern arises in response to experimental projects that transgress the boundaries of what is traditionally recognized as narrative, or might even be considered anti-narrative, such as William S. Burroughs' Naked Lunch (1959). More generally, the interactive, nonlinear, multilinear, and multimedia features of new narrative media present challenges to the plot-based definitions of narrative that were inspired by Aristotle's Poetics.

Consequently, some theorists argue that the field should be preserved (Gérard Genette, Gerald Prince) and that new fields should emerge to address emerging media, while others (Katherine Hayles, George Landow, Marie-Laure Ryan) argue that the field of narratology is impermanent and transmedial, and therefore should be expanded to include new narrative media.

\subsection{Wordplay}

At the centre of the debate about whether digital works are narrative is Aristotle's definition that equates narrative with plot and diminishes other elements, such as characters. As he states, "[t]he most important of these things is the structure of events, because tragedy is mimesis not of persons but of action and life" (Aristotle 59). The plot is considered "whole" when it consists of a beginning, middle, and end (60), which he adds should be presented in order, as "well-constructed plots ... should neither begin nor end at an arbitrary point, but should make use of the patterns stated" (60). For the same reason, Aristotle dismisses narratives that focus on a character as incoherent: a character 
may perform any series of actions, which are not likely to produce the desired linear narrative structure (61). Unity, he argues, is necessary for both coherence and the enjoyment of the audience. Given the interactive and multilinear structures of contemporary digital works, it is clear why they fail to meet Aristotle's standards. Yet, more interesting is the fact that this definition has been welcomed by literary theorists, as if literature had never experimented with time and nonlinearity.

In his introduction to $A$ Theory of Narrative, Rick Altman criticizes classical narratology for its limiting perspective of narrative (5); as he writes, "[f]rom Aristotle to Branigan, theorists insist that narratives have a beginning, middle, and end. In other words, narrative is not just a set of materials but is a quite specific method of organizing those materials" (17). Altman challenges the logic of Aristotle's basic plot structure by asking that if a narrative must contain these three points of delimitation, how is it possible that one can immediately identify a narrative without viewing the work through to its conclusion: "[i]f it is necessary to observe the entirety of a text in order to acknowledge it as narrative, then what is it that channel surfers are recognizing? How can narrative be at the same time something that is identifiable piecemeal and something requiring the experience of complete texts?" (17). Satisfied that he has debunked this view of narrative, Altman forwards a definition that relies on "following" characters. Unlike narratologists such as Chatman, Genette, and Mieke Bal who also highlight characters, Altman's theory of narrative does not rely on point-of-view or focalization (22). Altman's concept of "following" does not necessitate occupying the perspective of that character or narrator. The following of a character marks the distinction between the depiction of a scene and a narrative; it "is essential to the very category of narrative: wherever there is narrative 
there is following" (22). Altman's minimalist definition is, therefore, inclusive and transmedial.

Such inclusive narratologies, however, have been criticized, not only by classical narratologists, but also by Marie-Laure Ryan, who, in Avatars of Story, argues that "expansionist" approaches to narrative risk diluting the term until it becomes meaningless. As an example, Ryan singles out George Landow's work on hypertext as an offender (xv), which, she argues, includes experimental works that are explicitly antinarrative. Although Ryan does support criticisms of Aristotle's narrative in favour of a somewhat more inclusive and transmedial definition that accounts for drama, film, and games ("Toward a Definition of Narrative" 23, 26), she suggests that it is necessary for there to be a limit to this inclusivity. Ultimately, Ryan argues that debates about the definition of narrative are esoteric and generally unnecessary, as "judgments of narrativity are variable, and ... are not crucial to understanding” (31) or to identifying a narrative. Although these articulations do not definitively resolve questions about narrative, they do reveal that there exist varying and competing narratologies, and that the debate about what is or is not narrative is hardly settled.

As far as games are concerned, however, digital narratology is best equipped to address born-digital forms of storytelling - everything from hypertext to games. In their introduction to New Narratives, Ruth Page and Bronwen Thomas describe digital narratology as the study of the intersections of the theory and practice of digitally adapted texts and born-digital works, with an emphasis on narrative structure and interactivity. Given that the writing and copy-editing of print texts is now almost exclusively done on a computer, the idea of a definition of narrative that separates print and digital literature 
based on its production is untenable. In contrast to classical narratology, which aims to identify and delimit narrative elements, digital narratology is interested in questions concerning the interface and experience, the use of code to reimagine traditional narrative forms, and the implications of transmediation to consider what is "distinctive or innovative about 'new' storytelling modes" (3). As Marie-Laure Ryan outlines, these stories are interactive, reactive, multimedia, volatile, variable, and networked (Avatars 98). What makes digital narrative more dynamic than its print version is that it can be stored in a database, as this form allows what appears on the screen to transform as information is called from the database in response to user input. For Lev Manovich, the database and narrative are antithetical for the reason that each uses distinct methods to store and transmit information (225); yet, digital narratology maintains that there is much to be gained from rethinking narrative through new technologies. Digitally produced works and the databases and algorithms that support them create new and fascinating challenges for describing and analyzing narrative. Consider, for example, the project developed by Filmmaker Oscar Sharp and technologist Ross Goodwin that produced an AI screenwriter using complex algorithms that draw from large databases to create film scripts. As the description of the project explains, “[t]hey created 'Jetson' and fueled him with hundreds of sci-fi TV and movie scripts. Shortly thereafter, Jetson announced it wished to be addressed as Benjamin" ("Sunspring"). ${ }^{1}$ After Benjamin had written his first script, their team then worked over 48 hours to produce what the AI had titled Sunspring. Sci-fi no doubt is an apt genre for the emergence of what is likely the first AI screenwriter. Although the inhuman dialogue of the film may not be up to Hollywood

\footnotetext{
${ }^{1}$ See the description box for Sunspring.
} 
standards, the project imagines a future for a new kind of entertainment and authorship. In the team's most recent film, It's No Game (2017), starring David Hasselhoff, Benjamin addresses the looming threat of a strike by the Writers Guild of America, and humorously alludes to his own promising future.

Although AI film production might still be a distant future, the implications of this technology find particular relevance in game studies, given that games increasingly make use of technical features like randomly generated content and "intelligent AI storytellers" (e.g., RimWorld). That a virtual world is able to procedurally generate a narrative in response to the player's actions will have important consequences for our understanding of authorship. Janet Murray, Marie-Laure Ryan, George Landow, Espen Aarseth, and others address the effects of player agency and whether authors truly cede control to players in interactive works, but questions about whether an algorithm could be a kind of author and what this might mean for narrative have yet to receive the same attention. It is likely, given trends in the game industry, that it will be necessary to revisit the relationship between author, audience, and text in the very near future.

The field of digital narratology also offers important insights into the challenges of studying digital media. Rita Raley's “TXTual Practice," for instance, addresses the volatility of digital works. Although writing about digital works tends to present them as complete and contained textual objects, Raley reminds us that digital texts are not always as static, accessible, or permanent. Examining transient works, Raley shows the new challenges that these works create for digital narratology. Textual practices that rely on, for example, public SMS messages to create artworks, are "antihermeneutic" (20). As a result, they are resistant to archiving and preservation. While at one time, games were a 
static, physical medium that existed as cartridges and disks, the rise of networked consoles and online distribution platforms has destabilized and fragmented what was once a contained work. It is now expected that games will release patches, updates, addons, expansions, skins, new missions, storylines, characters, and other downloadable content (DLC). It is no longer uncommon for a game to make changes long after its release, and in fact producing such updates helps keep one's game relevant and featured on the hosting platform's main page. Such updates have become a hazard for writing about games, and will be addressed in more detail in Chapter 7 .

In tracing the development of narrative studies from classical (formalist) narratology to contemporary electronic literature, Page and Thomas note that the most important shift in the field is that "the apparent neutrality of narrative systems has in general been replaced by a critical narratology that draws attention to the ethics of storytelling" (6). No longer concerned with definitions and plot pyramids, contemporary narratology has moved "beyond formalism to explore issues of gatekeeping and access (as in who gets to tell what kinds of stories, and in what contexts), and to interrogate the increasing corporatization of cross-media franchises" (7). This new critical narratology addresses and emphasizes the impact of the stories that are produced and distributed over their structure and formal properties. Page and Thomas attribute this shift in narratology to media theory:

The critical and contextual breadth of digital narratology owes much to the tenets of new media theory, which concerns itself less with the stylistic or textual characteristics of new narrative forms than with the environments and social and 
cultural formations that produce and consume them, as well as the cultural uses

to which narrative practices may be put. (7)

Critical analyses of the hegemonic structures that influence the kinds of stories that are produced have obvious consequences for critiques of pedagogy's reliance on literary canons, but these critical approaches also find significance in thinking about the political implications of the stories that circulate in popular culture. Games in particular have been at the centre of debate in recent years as feminist scholars, such as Anita Sarkeesian, have challenged game content that tends to opt for narratives about white men that revolve around violence and rescuing distressed damsels ("Tropes vs. Women"). Such criticisms have spurred a culture war in games between feminists and supporters of the Gamergate movement.

Taking a digital narratological approach to game narratives provides an opportunity to apply the experience of the field to a new medium and to expand the field of narratology. There remains, however, the second obstacle to studying game narratives: ludologists reject the idea that games are a narrative medium, and see applications of narratology as an attempt by narratologists to colonize game studies.

\subsection{Ludology Vs. Narratology}

Although Janet Murray presented "The Last Word on Ludology v Narratology" at the meeting of the Digital Games Research Association (DiGRA) in 2005, the debate about whether games are a narrative medium continues to resurface. Given the success of major story-based single-player series like BioShock and The Witcher (2007), players might be surprised to learn that it is contentious to claim that games tell stories. As 
Souvik Mukherjee relates in Video Games and Storytelling, Roger Ebert, Steven Spielberg, George Lucas, and Will Wright (the creator of The Sims) have all made claims that player agency and narrative are incompatible (3); as Wright states, "games are not the right medium to tell stories" (qtd. in Mukherjee 3). And yet, each year, awards for best narrative are handed out at various major game awards ceremonies.

While this debate has had little impact on the industry, as the big budget games produced each year tend to replicate games that were successful in past years (Dymek 41), it has resonated with the independent game community. Jonathan Blow, creator of Braid (2009) and The Witness (2016), has stated that the meaning of his games emerges out of its rules and mechanics, rather than the narrative (Miguel Sicart, "Against Procedurality"). The ludology vs. narratology debate, however, is not fundamentally about the design of games, but rather revolves around key concerns about how games should be studied and who should study them. The ludological position argues that a game is its rules and mechanics, and that narrative serves only as the fantasmatic support for the player's actions, while the narratological position (supposedly) argues that games should be treated as narrative and analyzed in terms of plot and characters. In "Games Telling Stories?” (2001), Jesper Juul criticizes narratological approaches to games, and argues that rather than applying existing methodologies and theories to this new medium, "we must start afresh" and study games as distinct from other media. However, as previously illustrated, the branches of narratology interested in analyzing games are not interested in forcing games to conform to Aristotelian principles, but instead work to expand the study of narrative itself through new media. While Juul acknowledges that games and narrative share similarities, he argues that the aspects that separate the two are 
too great to overcome, and that a new approach is necessary.

Addressing arguments for approaching games as narrative, Juul's primary concern is that because we view narrative as the primary means for understanding the world, it prevents us from accepting other modes of representation; as he writes, "[t]he first argument is a compelling one, as it promises a kind of holistic view of the world: Since we use narratives to make sense of our lives, to process information, and since we can tell stories about a game we have played, no genre or form can be outside the narrative" (“Games Telling Stories”). In "Simulation versus Narrative," Frasca also argues for a ludological approach to games. As he explains, games vary from other media in that they use simulation rather than representation for expression. Frasca explains that we have become so entrenched in representation that it has become nearly impossible to imagine an alternative (224), especially given claims that our brains are hard-wired to work with narrative (223). Frasca defines simulation as the act of modelling a "system through a different system which maintains ... some of the behaviors of the original system" (223). Narratologists like Melanie Green, however, counter these claims about the difference between simulation and narrative by arguing that narrative itself is a kind of "simulation of the essential aspects of human life" (252), and the more realistic this simulation is, the more likely audiences are to be transported into its world. While there is merit to this idea that narrative as a representation form dominates our worldview, it is equally problematic to exorcise narrative from games on this basis, as audiences clearly recognize many games as narrative works. The defensive reaction to narratology by ludologists reveals that the narratology-ludology debate was a response to the fear of being colonized by disciplines like literature and film (Frasca, "Ludologists Love Stories" 
5). This impetuous reaction reduced the effectiveness of their arguments for considering the unique aspects of the medium; as Jan Simons (2007) writes, "[b]ecause in the heat of the debate ludologists sometimes seem to have lost sight of such subtle distinctions their arguments against narrative and narratology have often been unnecessarily unconvincing. Their arguments are ideologically motivated rather than theoretically grounded, and don't hold up against closer scrutiny" ("Narrative, Games, Theory"). Both ludology and narratology share much in common in terms of their structuralist approaches. In a later article, "Ludologists Love Stories, too: Notes from a Debate that Never Took Place," Frasca takes a more moderate position on narrative and games (though he does not revoke his earlier claims) and examines the origins of the ludology versus narratology debate and the misunderstandings that it perpetuated about game studies. As will be demonstrated throughout this project, neither the ludological nor the narratological perspective is wholly adequate to provide a comprehensive analysis of video games as a cultural object. Both the formal properties of the game and its narrative must be understood as interacting elements within a system, and not as mutually exclusive components of the user's experience. Examining Juul's claims about the incompatibility of narrative and games, however, provides important insights into the concerns that fuelled this debate and reveals some of the obstacles that narrative theory must overcome to address the changing mediascape.

\subsection{Against Transmediation}

Although Juul acknowledges that games and narrative share many similarities, he claims that existing narratives cannot be transmediated into games. Marjorie Siegel 
defines transmediation as simply "the act of translating meanings from one sign system to another" (455). Juul argues that this process is not possible for games, and he cites Atari's Star Wars (1983) as an example of such a failure; as he writes, "[i]f we imagine the title removed from the game, the connection would not be at all obvious. It would be a game where one should hit an 'exhaust port' (or simply a square), and the player could note a similarity with a scene in Star Wars, but you would not be able to reconstruct the events in the movie from the game" ("Games Telling Stories?"). At best, he avers, games based on existing narratives inject gameplay with a number of key plot points through cutscenes without developing the narrative or characters. While this example supports his account of narrative as a rationale for the player's actions, it is telling that he selected a game nearly two decades old at the time of publication, when there are a number of more recent movie franchises released as games — and even more recent Star Wars releases that would have sufficed. Juul might as well have chosen Atari's E.T. (1982), a game so legendary for its disastrous portrayal of Spielberg's film that a documentary was made about excavating the site rumoured and later proven to be the dumping ground of nearly a million unsold copies (Atari: Game Over 2014).

Moving beyond Star Wars, Juul furthers his challenge to narrative by taking Janet Murray at her word, and imagining one's experience of a fictional Hamlet on the Holodeck of sorts:

Even if we were to play only a single game session of a hypothetical game and end up performing exactly the same sequence of events that constitute Hamlet, we would not have had the same experience as had we watched Hamlet performed. We would also not consider the game to be the same object as the 
play since we would think of the game as an explorable dynamic system that allowed for a multitude of sequences. (“Games Telling Stories?”)

Here, Juul is correct: the experience of Hamlet as a holonovel would certainly differ from being a member of the audience at a performance of the play. It is likely, however, that viewing a performance of the play on opening and closing night would also produce a different experience. What Juul overlooks is that this difference in experience is not a failure or deficiency, but a characteristic of adaptation; as Linda Hutcheon writes, “[j]ust as there is no such thing as a literal translation, there can be no literal adaptation ... Transposition to another medium, or even moving within the same one, always means change or, in the language of the new media, 'reformatting'" (16). Likewise, Murray never intended the title to imply such a translation of Hamlet from one medium to another; as she writes, "[i]n trying to imagine Hamlet on the holodeck, then, I am not asking if it is possible to translate a particular Shakespeare play into another format. I am asking if we can hope to capture in cyberdrama something as true to the human condition, and as beautifully expressed, as the life that Shakespeare captures on the Elizabethan stage" (274). Each medium has strengths and weaknesses in its representation: a play performance would highlight the drama of the events; through closeups, a film enhances emotional intensity; a print-version offers attention to details about the nuances of language and allows time for close-readings.

Although it is likely not quite what Juul imagined, a game based on Hamlet now exists. To Be or Not to Be: That Is the Adventure (Tin Man Games 2015) was created by Ryan North, a Canadian writer known for his webcomics and graphic novels. The game is styled after Choose Your Own Adventure novels and allows players to experience the 
narrative as either Hamlet, Hamlet Sr., or Ophelia. After selecting a character, players can then choose to experience the work as intended or to explore North's creative take on the original, where players can make decisions that alter the narrative to imagine what could have been otherwise. Unlike Juul's account of transmediation, in North's game, players are not simply subject to cutscenes, but actively participate in the production of the narrative. The way that this particular example allows players to experience Hamlet in its more traditional or experimental form highlights how even when two works are similar, the ways that a work is adapted, interpreted, designed, and directed puts a new spin on the same story. Although reviewers are often concerned about whether a work is an authentic adaptation or "true to the original," the truth is that a work can never be a direct translation: there is always noise, different experiences and readings, that inform one's experience of a work, against which future adaptations will be compared. It might even be that we enjoy transmediated works precisely because they provide us with a different experience or take on a familiar work.

\subsection{Does Tetris Have a Narrative?}

In his critique of games as narrative, Juul addresses the problem that narrative approaches to games view narrative as an all-encompassing quality of a medium. Examining mechanically driven games like Tetris (Pazhitnov 1985) and Space Invaders (Taito 1977), he writes that “"Tetris - the movie' does not seem like a viable concept” (“Games Telling Stories?”). Similarly, although the promotional material for Space Invaders relate the story of an alien attack, the player's experience and interaction with the game's mechanics does not resemble a narrative work. Juul also writes that although 
the player may retrospectively relate the experience of playing the game in narrative form, the game itself is not a narrative. Likewise, even if one were to analyze the allegory concealed in the games mechanics, it still would not prove that games are narrative. Janet Murray, for example, argues in Hamlet on the Holodeck that Tetris contains a narrative about labour in capitalism:

Even a game with no verbal content, like Tetris, the wildly popular and powerfully absorbing computer game of the early 1990s has clear dramatic content. In Tetris irregularly shaped objects keep falling from the top of the screen and accumulating at the bottom. ... Instead of keeping what you build, as you would in a conventional jigsaw puzzle, in Tetris everything you bring to a shapely completion is swept away from you. Success means just being able to keep up with the flow. This game is a perfect enactment of the overtasked lives of Americans in the 1990s —of the constant bombardment of tasks that demand our attention and that we must somehow fit into our overcrowded schedules and clear off our desks in order to make room for the next onslaught. (143-44) Murray's analysis of the game resonates with the experience of the Post-Fordist worker, but whether it proves that Tetris is a narrative (in Juul's sense) is uncertain. If for the moment we dismiss Murray's analysis on the basis that this narrative is not immediately apparent to players, and concede to Juul's assertion that games are not narrative because mechanically driven games like Tetris are not recognized as narrative, then, logically, games are not narrative if they are not narrative all of the time. This line of thinking, however, neglects that other media contain content that is both narrative and nonnarrative. That players do not immediately identify Tetris as a narrative, even if it can be 
analyzed retrospectively as such, is not proof that games either are or are not a narrative medium.

Throughout the ludology vs. narratology debate, the usefulness of a comparative media approach is often overlooked, despite its ability to provides insight into game studies by drawing parallels to similar debates in other media. In this instance, consideration of the content of other media reveals that not all books (print or electronic) or films are narrative. Dictionaries, colouring books, atlases, and encyclopaedias are not narratives. A film like Andy Warhol's Empire (1964), an eight hour look at the Empire State Building in New York City from sun rise to sun set, is not a narrative work, nor are the numerous videos shared on various online platforms. Experimental or not, these books and videos fail to meet Rick Altman's conditions for recognizing a narrative, but at the same time, their existence does not disqualify print or film as a narrative medium. Likewise, games need not be excluded as narrative because of mechanics-based games like Tetris. Ludologists' arguments against narrative, which are tenuous at best (Simons), point not to poor logic, but instead to their eagerness for games to be understood as a distinct medium, and to isolate what it is that games offer that is not possible in other media. The failure to fully articulate this difference is the source for the continual reemergence of this debate, like the return of a repressed idea or desire.

\subsection{Resurrection}

In April 2017, Ian Bogost stirred up the debate yet again with his polemical article titled "Video Games Are Better Without Stories." No doubt designed to provoke readers, the game community erupted with responses ranging from close-readings of his 
commentary to outright scorn; as Bogost announced in a Tweet the day following the article's publication: "Both extremes of gaming are pissed at me-the mainstream traditionalists \& the fringe progressivists. And neither can see or hear the other" (@ibogost). His comment echos a similar but inverted frustration that Janet Murray levelled against ludologists in her "The Last Word on Ludology v Narratology," wherein she states that "[t]he 'ludologists' are debating a phantom of their own creation. ... Game studies, like any organized pursuit of knowledge, is not a zero-sum team contest, but a multi-dimensional, open-ended puzzle that we all are engaged in cooperatively solving." The debate, as she explains, never properly took place because there are no narratologists or narrativists who argue that games are a subset of narrative studies. Given the tension around the debate, it is no wonder that Bogost's article, as it was likely intended to do, refuelled the fire.

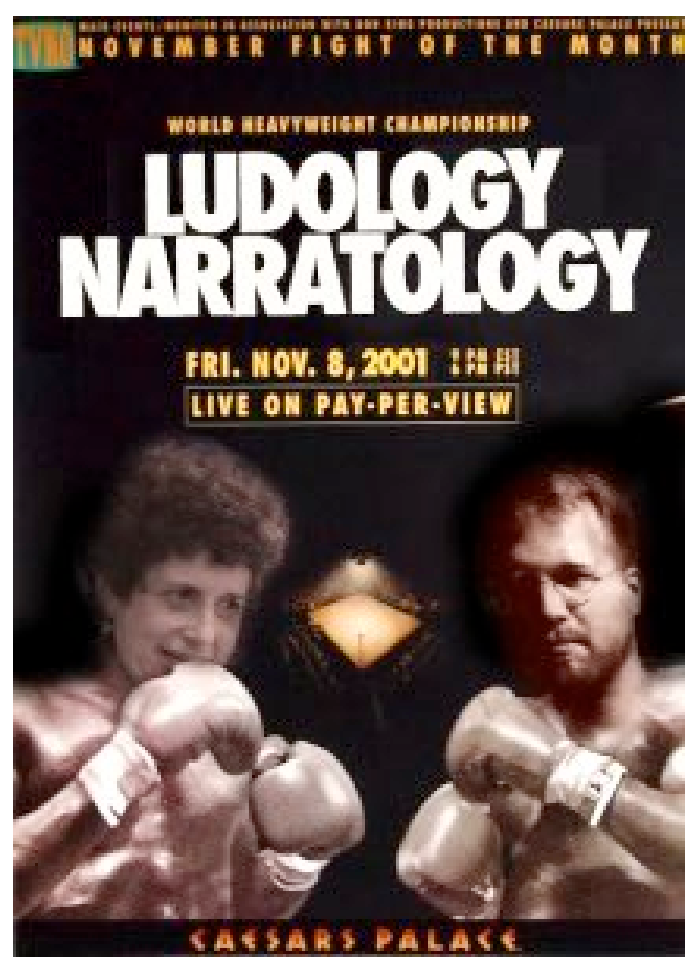


Fig. 2.1: an image by Ian Bogost that depicts Janet Murray and Espen Aarseth sparring to settle the Ludology vs. Narratology debate. "Videogames Are a Mess,” Bogost.com, 3 Sept. 2009

Despite its less than compelling appeal for ludology, Bogost's article posed important questions that offer insight into the cause of the return of this debate. The article reflects on the recent release of What Remains of Edith Finch (Giant Sparrow 2017) and asks, in spite of its compelling experience, "[w]hy does this story need to be told as a video game?" Bogost's analysis of the game is indebted to his own theory of "procedural rhetoric," the "practice of using processes persuasively" (Persuasive Games 3), which privileges a kind of computational rhetoric over a traditional linguistic form of expression in games. As such, this article positions itself on the ludology end of the ludology vs. narratology debate; consequently, it was viewed by many as a harsh criticism of the popularity of so-called "walking simulators." The term "walking simulator" was once used as a disparaging categorical tag for games that lacked traditional game mechanics and win-lose conditions; i.e., games that simulated players walking through an environment. This term has since been embraced by developers as a sub-genre of the first-person shooter (FPS), but while they adopt the aesthetics of FPS games, they reject their mechanics in favour of exploratory narrative environments: "[f]or these games, the glory of refusing the player agency was part of the goal. So much so that their creators even embraced the derogatory name 'walking simulator,' a sneer invented for them by their supposedly shooter-loving critics" ("Better Without Stories"). Because these games do not use violence as the primary means of interaction, they are often praised by feminist game critics for envisioning alternative modes of play and interaction. The walking simulator genre is particularly welcoming to both developers who want to 
use games as a form of expression and those who are new to playing games, as these games are often devoid of difficult challenges and rote memorization of enemy patterns. In asking why Edith Finch was made into a game (instead of a novel or film), Bogost's ludological position was read by some as a criticism of the walking simulator genre and the feminist values it represents. In their responses to his article, Brendan Keogh and Bianca Batti and Alisha Karabinus argue that Bogost's critique of narrative games is a patriarchal attack on recent trends in games that encourage inclusivity and representation of more modes of being in the world (@BRKeogh, Batti and Karabinus).

One particularly noteworthy example is The Fullbright Company's Gone Home (2013), which Bogost references in his article. Created by a small team of developers who had worked on BioShock, the game won many awards for its story that addresses LGBTQ issues about coming out to family and friends. Through Kaitlin, players explore an empty mansion and learn about her sister's difficulty growing up and coming out in the mid-1990s. Bogost notes its importance as a work in "a medium known for its unwavering masculinity" ("Better Without Stories"), but states that as an example of storytelling, it is mediocre. As Batti and Karabinus write, "Bogost rejects such stories because, he argues, '[g]ames' obsession with story obscures more ambitious goals anyway.' But what makes these other goals 'more ambitious'? More ambitious to whom? And who's to say that telling stories (especially those that are deprivileged, that are deemed marginal or unworthy) is not an ambitious goal as well?" The link between identity politics and narrative that Batti and Karabinus address is an important one that deserves repetition. Dismissing the value of games as a medium for diverse storytelling may not have been Bogost's intention, but it is an unavoidable consequence of his 
provocation. Although the focus here is not to examine the content of these stories in detail, it is imperative to acknowledge that there are real-world consequences for how stories are told, structured, and studied that this project will not ignore.

Although heavily criticized, Bogost's article is highlighted here because the questions that he raises provide a basis for thinking about why the ludology vs. narratology debate continues to re-emerge. Like Bogost's claim that Edith Finch "is pregnant with an unanswered question: Why does this story need to be told as a video game?," the unanswered question of Bogost's article is: Why do we keep rehashing this debate? Juul's analysis of the debate in "Games Telling Stories?" points to a belief that there is something different or unique about games, and Bogost's article takes an analogous position by noting the lack of ambition in using the medium for stories; however, precisely what sets games apart from other media remains unclear. Taken this way, his position on Edith Finch suggests not simply that another medium would be more appropriate, but more crucially asks what games offer that other media cannot. The ambitious goal of games may not be to tell stories, which are not unique to games; instead, the ambitious project is to explore the boundaries of the medium and its possibilities. Certainly, the ludology vs. narratology debate is well worn, and the article is problematic in all of the ways that Batti and Karabinus identify; and, yet, it drew significant attention and reanimated the debate. Definitively ending this debate is not within the scope of this project, as one can be sure, Bogost will write again. Responding to his criticisms, however, provides a launching point for considering the nuances of this debate which are necessary to analyze the role of narrative in communicative capitalism. 


\subsection{What's New about Narrative Games?}

Are the resulting interactive stories really interactive, when all the player does is assemble something from parts? Are they really stories, when they are really environments? And most of all, are they better stories than the more popular and proven ones in the cinema, on television, and in books? ("Better Without

\section{Stories")}

Holodecks aside, Bogost argues that games are better without stories because the stories told by games are not really interactive, nor are they really stories. Defining interactivity by what it is not obscures its meaning; following Bogost, it remains unclear what it would mean for a story to be interactive and precisely where narrative games fail to meet this goal. The term interactivity has been criticized for its imprecise definition. In Cybertext (1997), Aarseth outright rejects the term as meaningless, and accuses academics of uncritically accepting commercial rhetoric "with little concern for precise definitions or implicit ideologies" (48). Similarly, Janet Murray notes in Hamlet on the Holodeck, that interactivity is problematic and inconsistently applied; as she writes, "[b]ecause of the vague and pervasive use of the term interactivity, the pleasure of agency in electronic environments is often confused with the mere ability to move a joystick or click on a mouse. But activity alone is not agency" (128). Rather than rejecting the popular term, Murray refines its definition by distinguishing between activity and agency. Activity is often perceived as interactivity because, for some, using a game controller is an unfamiliar task that requires mental effort to recall which gestures and buttons correspond to on-screen actions, while for others, it is second nature. In 
addition to disrupting one's immersion in a game, a lack of familiarity with the controls is readily perceived as a kind of interactivity simply because manipulating the controller requires effort and heightened attention. In examining the ways that new media refashion old media, Jay Bolter and Richard Grusin explain that new digital media oscillate between immediacy and hypermediacy, between transparency and opacity. This oscillation is the key to understanding how a medium, fashions its predecessors and other contemporary media. Although each medium promises to reform its predecessors by offering a more immediate or authentic experience, the promise of reform inevitably leads us to become aware of the new medium as a medium. Thus, immediacy leads to hypermediacy. (19) Attempting to make games more immersive entails creating new controller configurations and clunky virtual reality (VR) headsets, but in trying to create a sense of immediacy, these devices also draw attention to themselves and players become aware of the experience as hypermediated. As Murray defines it, interactivity happens not with the physical device, but within the game when players are presented with opportunities for agency, which, she elaborates, "is the satisfying power to take meaningful action and see the results of our decisions and choices" (126).

In Avatars of Story, Ryan argues that when the term interactivity is used in relation to narrative, its meaning becomes unambiguous (99): the player will have to make choices. Although both Ryan and Murray provide a more polished account of interactivity through agency, it remains unclear exactly what it means to have agency by making choices. For instance, must the choices offered be meaningful, and, if so, how does one determine what qualifies as meaningful. If the player is given a choice between 
two options, but the consequences are only perceived to have an impact, is this action interactive? In other words, as the following chapter will examine in more detail, is BioShock's illusion of agency sufficient because the player believes it? This determination about whether or not a choice is meaningful could be defined by creating moments where events could have been otherwise. Referring generally to print texts, Prince's theory of the "disnarrated" is instructive; as he explains, the disnarrated consists of events in the text that are not narrated, but are nevertheless present (299), such as missed opportunities where readers imagine ways that events could have turned out, but did not. In this way, the disnarrated "provides one of the important means for emphasizing tellability: this narrative is worth narrating because it could have been otherwise" $(302)^{2}$. In electronic literature, readers often wonder what could have been if they had made different choices, and may even have the opportunity to return to the text to try out different scenarios. In this sense, players' choices can be considered meaningful if they create a moment that divides the events of the story. Yet, these moments can also be simulated through false choices, where a player is given a choice but the resulting outcome is the same. In these instances, players still wonder what might have happened if they had chosen otherwise, though the result is the same. The structure of such a narrative would appear similar to Marie-Laure Ryan's diagram of the flowchart, which illustrates an interactive architecture that is linear but where decisions affect the perceived story (Avatars 104). This structure is often used by Telltale Games (e.g., The Walking Dead) and other decision-based games to overcome technical limitations and to make the

\footnotetext{
2 The disnarrated is the opposite of Marie-Laure Ryan's virtual narration, which "describes a reflector and not events, but the reflected events usually belong to the actual world" ("Allegories of Immersion" 281).
} 
narrative appear more complex. In this sense, the act of "assembl[ing] something from parts" creates a sense of agency whether those choices have a real or perceived impact.

Bogost's second question serves to muddle the difference between stories and environments. Returning to the definition of narrative established in the Introduction, an environment is not a narrative, at least not until, Altman argues, a character is followed in that world: "[a] bird's-eye view of a city, or a detailed description of a battle, no matter how many individual actors and activities are visible, will provide at most the material for narrative. Not until the narrator begins to follow a particular character will the text be recognizable as narrative” (Altman 15-16). Games like Proteus (2013) or Mountain (2014) are examples of environments, where the player is given a perspective to observe the world; but it is not until the player is given a character to follow that a work becomes a narrative. A story is not "really an environment," it is the combination of environment and character. A game, therefore, can have both an environment and a story.

Apparently more important than whether games have stories or environments, Bogost's final provocation asks whether the quality of the stories told in games exceeds that of popular films, television, and books. It is unnecessary to spell out all of the ways that such a statement is problematic. Suffice it to say, it is elitist and a diminution of the important stories that games have shared. The test of time, a common measure for determining a great work, is challenging for a growing medium where the technology is outpaced every few years, making older games inaccessible to new generations, with the exception of exclusive digital platforms, like Nintendo's Virtual Console. Interactivity, or agency, does not inherently make a story "better," but it does introduce new creative, technical, and ethical challenges to storytelling that require further understanding. To the 
question, "why does it need to be a game," then, one could answer that creators are free to express themselves as they please in any medium they choose, but what underpins this question is the focus of the following chapters.

The question concerning what games offer storytelling is like that broad, often asked question in media studies: “what's new about new media?" For games, this question involves addressing what is new about how we produce and interact with these texts. Whether the stories of some game would be better suited as books and films, however, has little to do with games as media. This question, however, drives ludologists to set games apart from narrative media like film and books; as Markku Eskelinen writes, "[t]he old and new game components, their dynamic combination and distribution, the registers, the necessary manipulation of temporal, causal, spatial and functional relations and properties not to mention the rules and the goals and the lack of audience should suffice to set games and the gaming situation apart from narrative and drama, and to annihilate for good the discussion of games as stories, narratives or cinema" ("The Gaming Situation"). In a certain way, Eskelinen is right to say that games are not narrative, they are media; as such, they are no more narrative than the book which is often presumed to be inextricable from narrative, but, as previously addressed, one must not forget that books are not narrative all of the time. Arguments for the opposition of games and narrative do hold up against scrutiny. Games are media, and the content of media is often, but not always, narrative. When games and storytelling are combined, however, the affordances of the medium permit forms of agency, interaction, and exploration that do not simply reduce games to narrative, but create new forms of narrative that require new approaches and terms for analyzing and understanding these 
works. The following chapters will address issues of agency and experience to analyze how industry trends shape the stories that are told and the political and ethical consequences and complications that result. The next chapter draws on the BioShock series to focus on agency in narrative games, in order to examine the player's experience of free will, and how developers create a coherent narrative experience while maintaining the player's sense of control. 


\section{Section II Agency}

\section{Chapter Three: Decision Systems}

\subsection{The Myth}

Video games are often promoted as a medium for multilinear storytelling that allows players to make meaningful choices that affect narrative outcomes. Games like BioShock (2007), The Walking Dead (2012), and Mass Effect (2012) promise players compelling interactive narratives that adapt to players' decisions to create more personalized stories. The consequences of these decisions, however, do not always live up to the hype that players expect based on advertising claims. Such disappointment is explicitly manifest in the lawsuit that was launched against BioWare for the conclusion of Mass Effect 3. Fans argued that the game failed to meet advertising claims on the game's original packaging that stated that the "[d]ecisions you make completely shape your experience" (Mass Effect 3). ${ }^{3}$ The development of a truly dynamic and adaptive narrative has been an aspiration of storytelling long before its popularization in games; it is a theme that was previously explored in works like Jorge Luis Borges' short story "The Garden of Forking Paths" (1941), "Choose Your Own Adventure" novels from the 1980s, and later hypertext fiction. While narrative games have not yet fully realized this goal, many are working toward creative solutions that go beyond branching paths in a way that comes closer to satisfying the desire that Marie-Laure Ryan describes in "Beyond Myth and Metaphor" to create a finite text that contains an infinite number of stories (588).

\footnotetext{
${ }^{3}$ This claim appears on the back of the original packaging for Mass Effect 3, but is no
} longer present on more recent copies of the game. 
Such a mythical text provides a horizon for a future of storytelling where narratives are produced by their readers rather than their authors. While the novel has always been capable of non-linear storytelling, it is limited by its physical form, as its entirety can be seen and read. The digital, however, exceeds its physicality, which leaves readers and players uncertain about whether they have seen and heard everything. Advancements in digital technologies, like the complex algorithms for procedural generation and sophisticated AI, feel like a step toward this mythological text, whether its actualization is possible or not. The BioShock series has been particularly influential in its exploration of the limitations of this myth, and has inspired developers to consider new approaches to player agency. Beginning with a brief comparison of BioShock and Bioshock Infinite (2013), this chapter examines the series' approach to narrative and agency. By putting these two works into dialogue with one another, this chapter also forms a debate about the limits of player agency to argue that while a fully responsive narrative may be desirable, handing over narrative control to the player limits the critical power of games.

\subsection{Branching Narratives}

Along with its playful engagement with utopian tradition and American history, the first iteration of the BioShock series is also well-known for examining the limits of agency and free will. As Jack, the player explores a dystopian underwater environment that they must also escape. With the help of Atlas, a faceless voice transmitted by radio, the player must make difficult choices to navigate the maze-like space and survive the hordes of enemies. At the end of BioShock, however, players learn that many of the 
choices that they had made throughout the game were the result of Atlas' special command ("Would You Kindly") that ensured the player's compliance. As Jessica Aldred and Brian Greenspan rightly state, "[t]his revelation renders any of the 'choices' you thought you had made through the course of gameplay ... purely illusory, and the game ultimately mocks your helplessness at both a procedural and a narrative level" (490). Through the player's interaction with Atlas, the game cleverly crafts a world that challenges whether player choices are meaningful in a context where all possible outcomes are always-already determined by the developer. This limit on freedom is most apparent in the player's confrontation with Andrew Ryan, where the game removes the player's control over their avatar and they are forced to watch as their avatar murders him. Through this shift in control, the game reveals a limit to the player's agency, as if to invite players to question whether their agency is real or perceived, to which, despite its mockery of the player, it appears to respond in the negative. Despite industry trends that emphasize complex multilinear narratives, BioShock demonstrates that creating the perception of multilinearity is just as effective as the real thing.

As studious players will note, however, BioShock concludes with three possible alternate endings. Each ending foretells a different future of Rapture based on the player's treatment of the Little Sisters, which they may choose to save or harvest at different moments throughout the game. In an interview, Ken Levine explains that the game's multiple endings were never a part of the original vision for BioShock, but were "requested by somebody up the food chain from [him]" (Sinclair). Ultimately, these alternate endings are superfluous in that they do not shape the narrative in response to the player's intervention in the game, but are instead clips that offer broad projections of the 
future that are shown when the player completes the game. It would seem that the endings serve to placate corporate demands to boost sales. This critique, however, does not mean that these endings are not meaningful for players, but rather demonstrates that these apparently player-driven outcomes represent the very branching narratives that the game itself critiques: the player's choices only exist within the constraints set by the developer - even when the outcomes are multiple. BioShock's challenge to player agency echoes Gilles Deleuze's analysis of freedom in the society of control; as he writes, “[c]ontrol is not discipline. You do not confine people with a highway. But by making highways, you multiply the means of control. I am not saying this is the only aim of highways, but people can travel infinitely and 'freely' without being confined while being perfectly controlled. That is our future" (322). In a similar way, games offer the freedom of an agency that is always controlled.

\subsection{Infinite Worlds}

In its third iteration, BioShock Infinite (2013), the series takes a more nuanced approach to agency by considering more fundamentally what kinds of narrative models are made possible by games as a medium and whether the choices games can offer are meaningful. Although the characters, setting, and narrative were completely redesigned, both BioShock and Infinite share many similar themes and ludological elements. The player is equipped with the same weapons, plasmids, and EVE (now called salts and vigors), and the narrative is delivered through familiar audio diaries (voxophones) and propaganda films (kinetoscopes). Yet, while the mechanics and fragmented delivery of the narrative remain the same, Infinite differs in that it does away with the divergent 
endings that were a staple of the series. In Infinite, players discover that their choices have no effect on the game's conclusion, a rather unexpected lesson from a series that was known for its alternate endings. Throughout the game, players are offered choices in what appear to be key moments; the option to spare or to kill Slate (a somewhat minor character with whom you have a confrontation); to draw their weapon or give a nonplayable character the benefit of the doubt in a tense situation; and, perhaps the most memorable and enigmatic, the choice between the bird or the cage. The meaning and significance behind this choice is as mysterious to players as it is to anyone learning about this choice for the first time now. The choice between a bird or a cage symbolizes ideas about free will and determinism, which is no doubt significant for a series known for its critique of agency. Yet, as the player concludes the game, it quickly becomes apparent that none of the player's decisions have had a lasting impact, as the game offers only one conclusive ending.

In his article on "Meaningful Choice in Games," Brice Morrison states that in order for game decisions to be meaningful, they must adhere to four conditions: awareness, consequences, reminders, and permanence. In other words, players must be aware that they are making a choice that has repercussions on gameplay, they must be reminded of their choice, and they must not be able to reverse their decision. Infinite, however, fails to conform to Morrison's second category that requires that choices have consequences. Yet, players still experience these decisions as meaningful, in part because of the expectations that the series has set for players. In determining what "counts" as a meaningful decision, it is important to consider that experience and theory do not necessarily coincide. Although players discover that their decisions never "matter" in the 
way they expect, Infinite's conclusion is hardly lacking in sophistication. Players are shown a world (or many worlds) where all narrative outcomes are possible and simultaneous, each variation manifest as a lighthouse (i.e. an alternate world or timeline). The player's choices cannot affect the events of the game because, in this world, events occur in a particular way. The representation of these alternate worlds as lighthouses is apt given their signification as beacons to guide travellers; a lighthouse implies that the destination ahead requires a restricted path, as opposed to a ship's typically freeform movement through the open water.

This vision of intersecting dimensions shares much in common with Marie-Laure Ryan's account of hypertext fiction as an Aleph, a reference to the short story by Jorge Luis Borges. In this story, the Aleph is described as "one of the points in space that contain all points" (280) By looking into the Aleph, one is able to simultaneously see everything in the universe from all possible angles. In "Beyond Myth and Metaphor," Ryan employs the story of the Aleph to mythologize the belief that by randomizing the sequence of narrative events, hypertext can create a finite text that contains an infinite number of stories. As Ryan explains, "the pioneers of hypertext dreamed their brainchild as the ultimate literary work, the sum of all possible narratives, the only text the reader will ever need because its meaning cannot be exhausted" (587). Ryan describes this vision of hypertext as a myth for the reason that the reader's experience of such a text does not produce infinite stories, but rather the reader will instead "construe a global representation over many sessions" (590). With each reading of the text, the reader will adjust and amend the narrative to account for new information. This description of hypertext narrative provides a compelling model to consider in relation to the ending of 
Infinite, where in the final moments of the game, Elizabeth transports the player outside of the city of Columbia to somewhere that resembles an Aleph: an area that contains an infinite number of lighthouses that lead to an infinite number of alternate realities. These realities are all similar yet different, each with constants and variables. Elizabeth explains to the player that in every world, “[t]here's always a lighthouse. There's always a man. There's always a city" (Infinite). Regardless of the choices made by players, they will always be lead to a singular, conclusive ending. Booker's search for Elizabeth will always play out exactly the same with only minor variations for the reason that in this dimension, that is how events took place. The events of the game play out in one of an infinite number of possible worlds, and as a result, the player's decisions are only variables within a larger predetermined series of events that were set into motion by Zachary Comstock, the Father of Columbia.

With the aid of his physicist in residence, Rosalind Lutece, Comstock discovers how to open portals that allow him to move freely through time across dimensions. This ability not only enables him to bring the music of Cyndi Lauper and the Beach Boys to the year 1912, but also to "predict" the future, and as a prophet he gains the political influence needed to build his floating utopia. As a result of the effects of transdimensional travel, Comstock becomes unable to produce an heir; in order to correct this complication, Comstock travels to another reality where he persuades an alternate version of himself, Booker DeWitt, to surrender his daughter, Anna, in exchange for erasing his debt. This act, as Comstock is well aware, sets into motion a dangerous threat to his utopia, as Booker will later be sent to Columbia by the Luteces, who seek to undo the harm that their work has made possible. The Luteces travel to Booker's dimension years 
later and offer him a deal: "Bring us the girl and wipe away the debt." Booker is instructed to go to Columbia to retrieve Elizabeth — whom he no longer recognizes as his daughter.

Booker's arrival in Columbia, however, represents not only a threat to Comstock's utopia, but also a threat to the game's seemingly multilinear narrative. Through its decision-making moments, Infinite signals the promise of a branching narrative; yet, this promise is threatened by Booker's inadvertent quest to undo Comstock's plan. This tension between linear and multilinear narrative is played out between Booker's doubled presence in the game as both himself and Comstock. If Comstock, as a figure who is not restricted by time and who possesses the ability to alter future outcomes, represents the promise of new narrative media to create truly interactive and multilinear game worlds, Booker, by contrast, plays the role of the unwitting Oedipus, whose unalterable and linear path is laid out before him. In spite of Comstock's efforts to manipulate the future, as Elizabeth explains, "[i]t will happen all the same ... Because it does. Because it has. Because it will" (Infinite).

While one could draw a parallel between the triangle of characters with the events of Oedipus Rex, such an analogy would miss the point. That Oedipus murdered his father and married his mother, after all, is only a consequence of Oedipus' failure to understand the message of the oracle. As Jonathan Lear writes in Open Minded (1998),

Oedipus' fundamental mistake lies in his assumption that meaning is transparent to human reason ... It is the classic scene of fulfilling one's fate in the very act of trying to escape it. But this scenario is possible only because Oedipus assumes he understands his situation, that the meaning of the oracle is immediately available 
to his conscious understanding. That is why he thinks he can respond to the oracle with a straightforward application of practical reason. Oedipus' mistake, in essence, is to ignore unconscious meaning. (29)

In the same way, Booker's mistake is to overlook the unconscious meaning in the message: "Bring us the girl and wipe away the debt" (Infinite). These are the words of Booker's oracle, which he takes to mean that he must go to Columbia to retrieve Comstock's heir, Elizabeth. The true meaning behind these words, however, is a traumatic reality that his conscious mind cannot accept: Booker has already brought them Elizabeth, who is Anna, Booker's daughter. To account for this significant cognitive dissonance, Robert Lutece theorizes that when a subject moves from one dimension to another, the mind must compensate and create congruence in one's memories to account for the shift in dimension; as he explains, "[t]he mind of the subject will desperately struggle to create memories where none exist" (Infinite). When these false memories fail to conceal the truth, when the unconscious emerges, the individual exhibits the telltale psychosomatic symptom: a nosebleed. Booker's discordant memories are not subject to time; as Sigmund Freud asserts, "[t]he processes of the [unconscious] are timeless; i.e. they are not ordered temporally, are not altered by the passage of time; they have no reference to time at all" ("The Unconscious" 582). As Booker's encounters with Comstock reveal, when incongruent memories are activated by associated words (e.g., when Comstock mentions Anna), Booker's linear self-imposed narrative of his memories is disrupted, which induces a nosebleed. If there were any doubt about Booker's signification of linearity, the developers reinforce this reading by introducing a new ludic mechanic to Infinite's gameplay: the skyline. While travelling through Columbia, Booker 
is on rails, an expression used to describe, and sometimes criticize, linear storytelling. Despite its promises of agency through the choices it offers players, the use of the skyline undermines the player's freedom: what better way to manifest linearity than to put the player on a track.

Booker and Comstock not only enact the tension between linear and multilinear storytelling, but the resolution of their story challenges the promise that games, and digital narratives in general, can create truly dynamic and adaptive playable narratives. Comstock's attempts to alter his future (and escape his past) by travelling across dimensions is ultimately put to an end by Booker's death at the moment when their stories diverge: when Booker either accepts his baptism and becomes Comstock, or rejects the baptism and remains on his troubled path. As Elizabeth states, "[i]t all has to come to an end. To have never started. Not just in this world. But in all of ours" (Infinite). The divergent worlds that Infinite creates illustrate the impossibility of the Aleph as a functional narrative structure; as Marie-Laure Ryan argues, readers, or players, will interpret a collection of events into a single narrative with constants and variables, rather than experience each playthrough as distinct narratives.

In "BioShock: Complex and Alternate Histories," Ryan Lizardi offers an analysis of Infinite and its approach to alternate histories. By comparing the singular conclusion of Infinite with the forking paths of the earlier games, he concludes that,

If we truly do not know what we have until it is gone, then the absence of choice points us more directly towards recognition of its importance. When the ludic 'forking paths' are taken away from players of Infinite, but the narrative keeps pointing towards their significance, the resulting game experience tells players to 
value alternate histories, or at the very least a strong consideration of history as multi-causal.

For Lizardi, the absence of Infinite's branching narrative paths tricks players into realizing the value of alternate endings and histories by making their absence felt. While the sentiment behind his conclusion is admirable, this approach fails to fully consider the ludological and narratological dimensions that reveal these choices and branching paths to be very much present, much like alternate histories. While the player may not be able to access Infinite's branching endings, they are present in the form of alternate worlds contained in lighthouses. However, one's access to those histories is already determined by whoever is in charge: in this case, the developer.

Ultimately, the BioShock series critiques the novelty of branching paths as a narrative structure, and what players learn is that their choices are always variables along a path set by the developer. The possible outcomes of the earlier games were always predetermined, even if they were multiple. This is why when Elizabeth opens a tear in space and time that brings us to the underwater city of Rapture, Infinite reveals that Rapture, too, is a lighthouse. Through its fascinating ending, Infinite offers a clever ruse that both explains away the player's inability to shape the outcome of the game, and illustrates the inherent technical difficulties in creating narrative worlds that offer players an active role in shaping the story. In a certain way, the ending of Infinite imagines a possible (or impossible) future for storytelling in games, while simultaneously signalling precisely what it cannot achieve: a fully adaptive narrative that offers truly meaningful choices. Although the player's decisions lead to alternate endings, these outcomes are not unique to the player and were always predetermined. At a technical level, Infinite 
suggests that the problem with creating a narrative structured like an Aleph is the significant amount of content required to sustain such a dynamic world; as players make choices, the amount of content needed to account for decisions grows exponentially. The choices offered in Infinite are structured to create the illusion of agency by obscuring their consequences. Although Booker will make different choices about his future in another world, in this dimension, he did not, and therefore the player is forced to remain on the rails of the game's predetermined path. Consequently, these possible worlds are inaccessible to the player, as each exists only as a separate dimension. Or, rather, each exists as its own self-contained game or downloadable content, as each addition to the BioShock franchise creates another lighthouse.

\subsection{After BioShock}

Although it is a fiction, the Aleph represents a desire for dynamic and responsive stories and as a myth, it has become a point of aspiration for many developers. No doubt, this desire is precisely what motivates one of Ken Levine's projects that focuses on the development of what he calls narrative LEGO. Having exhausted the limits of linear storytelling, Levine is designing a system that will generate "replayable, player driven narrative gameplay" (Levine). As he envisions it, this system will work by creating an emergent narrative through moments of interaction with characters that affect gameplay events and dynamics. It is clear from his talk on narrative LEGOs at the 2014 Game Developers Conference that the end goal is to hand over authorial control of the narrative to players. Robert Jackson argues that this project "may achieve what it really means to live in a technological society: to construct an ecology not where we are bestowed 
surprising freedom, but where a system decides on our actions in such a way that are [sic] 'ultimately freeing"' (305).

Clearly if the BioShock series and its descendants continue to explore the possibilities of player agency, they will face similar design challenges. In a number of ways, BioShock Infinite already clashed with player expectations about the series. A quick Google search reveals countless queries about Elizabeth's enigmatic bird or cage pendant and whether or not this decision would produce multiple endings like those offered by the earlier games in the franchise. Players expect that the mechanics and lessons taught in a game will carry over to sequels, and when these expectations are disrupted (whether as metacommentary or merely due to bad design), developers risk alienating their audience.

Although Telltale's linear narrative structure may not ultimately diverge significantly from that offered by the BioShock series, it demonstrates an effective strategy for making player choices meaningful without compromising the length of the game or creating a structure that is too sprawling and complex to produce. It also succeeds in providing meaningful choices, even if they are somewhat limited, in a universe that is already well established. As Toby Smethurst and Stef Craps point out in "Playing with Trauma: Interreactivity, Empathy, and Complicity in The Walking Dead Video Game," even false choices that have no bearing on the outcome make the story appear more complex without expending much effort. However, for both transmedia works and sequels, the player's interactions are always informed by existing knowledge of the franchise, which may place limits on opportunities for agency.

In the post-BioShock era, the series' critique of agency has been picked up and picked apart by a number of games that follow in its wake, such as Orthogonal Games' 
The Novelist (2013) and Telltale's The Walking Dead (2012). These developers have taken new approaches to narrative systems that attempt to move beyond the technically challenging form of branching stories. These games challenge BioShock's limited view on agency and use creative solutions to rethink meaningful play. The following chapter will examine these games to analyze and evaluate the role of agency in shaping their narratives. 


\section{Chapter Four: New Approaches to Narrative Agency}

\subsection{Algorithmic Approaches: The Novelist}

Following his roles as a senior designer on BioShock 2 and consultant on Infinite, Kent Hudson released The Novelist, a game that attempts to employ an algorithm to create a meaningful narrative experience. Dissatisfied with the storytelling methods of BioShock, Hudson hoped to create a way for players to make meaningful choices without relying on traditional branching narrative structures. In his talk "The Game Is the Message," delivered at the 2014 Game Developers Conference, Hudson argued that "game stories are on the wrong path, [and are] telling linear stories that would be better as films or novels. Even worse, most game stories don't take advantage of the interactivity of [the] medium."

The problem that Hudson identifies with game narratives is evident in projects like those emerging from the teaming up of Telltale Games and Lions Gate Entertainment, who are working to create what they call a "Super Show," an interactive television series that combines ludic elements with a scripted television format. ${ }^{4}$ Although the details of this project are vague, its description suggests that the product might fit into the genre of full motion video (FMV) games, which includes titles like Don Bluth's Dragon's Lair (1983) and Late Shift (2017). Hudson's game The Novelist, however, attempts to create a game environment that gives players control over the direction of the narrative by using an algorithm rather than scripted narrative branches. The game tells the story of the Kaplan family as they move into a vacation home with

${ }^{4}$ According to Telltale's website, the first iteration of this series is due to premier in 2019. 
hopes that the father, Dan, will be able to work on his novel and the family will strengthen their relationships. As a ghost, the player observes the family and the difficulties that arise during their stay, such as a death in the family, Tommy's school troubles, and upcoming deadlines. With each crisis that arises, the player is given a chance to learn the thoughts and concerns of each family member, and while the Kaplans sleep, the player must tell Dan how he should resolve the family's conflicting desires. If players examine the thoughts of each character sufficiently, they will be able to uncover one compromise for another character. For example, when they first arrive at the house, Dan feels that he needs to focus on his work, but his son, Tommy, asks to go to the beach, and his wife, Linda, wants to spend an evening together. During the night, the player will influence Dan by whispering which action to take, along with a compromise for one character. In this instance, the player may choose to let Dan work all day, but compromise with Linda by spending a short time with her in the evening. This resolution will leave Tommy disappointed. Players must navigate the challenges of marriage, work, and parenthood by making difficult decisions and compromises in an effort to keep the family together; it is a story in which, Hudson says, "the player makes influential decisions that create a unique narrative that is not prescribed by a designer."

Rather than using branching narratives, Hudson's algorithm uses a scoring system that determines the outcome of character interactions and compromises in order to give the player a more dynamic influence over the outcome of the game. Inadvertently, however, the game's decision system makes it difficult for players to achieve an optimal outcome. After the release of the game, Hudson found that reviewers were disappointed by the inability to get a happy ending for all of the characters, as each decision and 
compromise necessitates that one family member is always disappointed; consequently, as the game progresses, it becomes difficult to balance decisions evenly across each of the characters. Though Hudson did not intend to create a game that conveyed a depressing message about family life, he acknowledges that altering the system would make the game too easy and would no longer accurately reflect the challenges of negotiating the sacrifices and demands of family life (Hudson).

Hudson's system is an excellent contrast to BioShock's limited narrative structure. Although it may not be as all-encompassing as an Aleph, it successfully gives the player control over the narrative; as Hudson states, “the player isn't just choosing predefined paths along an overall linear flow; the choices actually create new narrative situations." The Novelist satisfies one's desire to interact with stories by allowing players to intervene in ways that one wishes one could in other media — for example, by yelling at the television when characters make undesirable choices. Hudson's game relieves this tension by giving the player control over key moments in the story. Unlike a branching narrative that creates a moment that divides the story, each chapter presents an opportunity to shape the characters' relationships. Rather than producing a good or bad ending, the game offers players a dynamic narrative that changes in response to the consequences of their decisions. The concluding synopsis of the player's choices reveals the reception of Dan's novel and the future of his career; if Linda successfully pursues her career as an artist; and whether Tommy will thrive or struggle in school.

One disadvantage of Hudson's system, especially for indie developers, is that this model requires a significant amount of content to account for each narrative possibility, which can affect the length of the game. The Novelist is about two hours in length, and 
consists of nine chapters and thirteen outcomes, which totals over a hundred possible scenes. This limitation remains a factor for studios backed by large publishers, as any increase in the number of narrative situations will exponentially increase the amount of content needed to complete the game. Although this model is difficult to adapt for more moderate length games, other developers are working on solutions for larger games by allowing players to shape character dispositions, as seen in Telltale's The Walking Dead, or by enabling players to explore all possible outcomes in limited scenarios, as demonstrated in The Stanley Parable. Each approach has strengths and weaknesses, but all attempt to give the player a degree of freedom over the world in which they are placed.

\subsection{Who Will You Become?: The Walking Dead}

In 2012, Telltale Games released its take on The Walking Dead: a game that, like the comic and TV series, is centred on making difficult decisions. In Telltale's version, players' decisions affect the development of the narrative; as the prompt at the start of the game states, "[t]his game series adapts to the choices you make. The story is tailored by how you play" (The Walking Dead). These choices, however, do not always matter in the way that players expect. Although the game has overwhelmingly positive reviews, some reviewers criticized the game for failing to have consequences for the choices it offers. ${ }^{5}$ While the decisions that players make do not allow them to stray from the largely linear direction of the game, it does not mean that these choices are insignificant. Rather than creating diverging storylines as players expect, decisions shape the personality of their

\footnotetext{
${ }^{5}$ See store.steampowered.com/app/207610/
} 
character, Lee Everett, and his relationships with other characters. Players are introduced to Lee as he is on his way to prison. Once a university professor, Lee was convicted for murdering the man who had an affair with his wife; however, thanks to the chaos of the zombie outbreak, Lee never makes it to prison. After players take control of Lee, they soon meet Clementine, an eight-year-old girl who is alone now that her babysitter has been bitten, and her parents remain away in another state. Unable to leave her behind, Lee becomes Clementine's guardian.

By focusing on characters rather than plot, Telltale's developers sidestep the most significant problem of creating adaptive narratives which was addressed in the discussions of BioShock and The Novelist: namely, the need for significant amounts of content to support branching narrative paths. Dialogue options are much more flexible, and do not necessarily require the creation of new scenes and events to account for the player's choices. In part, these challenges explain why the endings of BioShock are projections of the future beyond the game's ending, rather than scenes embedded throughout the narrative that reflect the player's direct intervention in the world. Telltale's character-based approach is able to shape the narrative of The Walking Dead in response to the player's decisions in a way that influences Lee's personality to determine whether he is honest, trustworthy, and a team player, or self-interested, secretive, and aggressive. These qualities have consequences when players must manage the group's resources and mediate conflicts: for instance, whether or not players reveal to other characters that Lee is a convicted criminal and that Clementine is not his daughter provides the basis for many of Lee's relationships and will determine who will leave or 
stay with the group throughout the series. This approach to player agency, as this section will explore, is not without its limitations.

The aim of Telltale's decision system is made most explicit in a promotional video for the second season of the series which asks, "Who Will You Become?," and directs fans to the Twitter hashtag \#MyClementine. The advertisement makes clear that the player's choices will influence who Clementine will become as she grows up in the midst of the zombie apocalypse. However, the answer to Telltale's question is complicated given the already complex relationship between the player and the avatar, as well as the game's significant corpus of franchise material that informs both the developer and player. The Walking Dead universe, which began as a comic book by Robert Kirkman, Tony Moore, and Charlie Adlard, has since expanded into two television series, several novels, tabletop, and digital games. On their panel "When Story is the Gameplay" at the 2015 Game Developers Conference, Telltale developers noted that one of the greatest challenges that they faced when creating the game was dealing with the significant amount of canonical baggage. The developers wanted to create an exciting experience, while simultaneously staying true to the spirit of the comic. To solve the problem of retelling familiar stories in new ways, Telltale introduced all-new characters into a format that they describe as an "interactive scripted narrative" (Bissell). Rather than create a traditional action-oriented franchise tie-in, developers wanted a narrativedriven game punctuated by meaningful choices that shape the player-avatar relationship. 


\subsection{Players and Avatars}

In "Playing at Being: Psychoanalysis and the Avatar," Bob Rehak theorizes this player-avatar relationship through Jacques Lacan's theory of the mirror stage. Rehak argues that, through the player's interaction with the avatar, "video games 'reflect' players back to themselves" (104). This reflection, however, is only ever imperfect. The discordance between one's image and body structures and divides the subject's identity: one is always split between who one believes oneself to be and the incongruent image that is reflected back in the mirror. This reflection is a specular image, an image that is "simultaneously oneself and other" (Evans 190). Unlike other media, Rehak argues, the interactive nature of video games offers players the opportunity to control and direct the actions of the avatar. While in cinema, identification is mediated through the camera that fixes our gaze (103), games put the player in an unusual position to both play and watch themselves play (119). By merging spectatorship and participation, games transform the subject's relation to the image. It is problematic, however, to apply an unwieldy term like identification to this experience.

Identification, like many terms in game studies, is often applied without precise definition (Papale 2014; Shaw 2015). In "Beyond Identification," Luca Papale reserves this term to describe a particular relationship between player and avatar where "identification grants agency to the video game and its characters, that is the ability to operate on, to influence, to manipulate, to persuade the subject player, at the point of altering the player's personality" (3). More commonly, players experience feelings of 
empathy, sympathy, projection, and detachment rather than identification. ${ }^{6}$ In instances of sympathy or detachment, Rehak's application of the mirror stage would be unsuitable. Although the player is engaged in a kind of mirroring through the figure on the screen and their ability to manipulate its movements, their connection to the character is more like a cursor.

When given the opportunity, players spend a lot of time designing, developing, and upgrading their avatars, shaping them into a kind of ideal-ego. The avatar stands in for the player as an uncanny double, one that differs "from us through their ability to live, die, and live again" (107), which allows players to take an active role in the constitution of their identity. That avatars can be "paused, erased, or restarted" (107) is significant because it facilitates the endless repetition that drives desire. Rehak argues that players stage and replay the incongruence in their own subjectivity through the avatar: "video games seem to enact the fort/da game. If our unity is itself a misrecognition, then the video game, for all its chaotic cartoonishness, may constitute a small square of contemplative space ... in which we toy with subjectivity, play with being" (123). In Beyond the Pleasure Principle, Freud illustrates the relationship between repetition and desire through the story of a game that his grandson played. Distressed by his mother's daily disappearances, his grandson created a game, using a spool and some thread, in which he restaged the painful experience of his mother's departure by throwing the spool, and the joy of her return by reeling it back. In his analysis of this game, Freud describes

\footnotetext{
${ }^{6}$ The terms avatar and character are used to distinguish between figures that are customizable "blank slates" and those that are given predefined traits with limited customizations; for example, the player's avatar in Mass Effect and the character of Mario in Nintendo's franchise.
} 
that, "[a]t the outset [his grandson] was in a passive situation-he was overpowered by the experience; but, by repeating it, unpleasurable though it was, as a game, he took on an active part" (15). Rehak argues that, in the very same way, games allow players to take an active role in the constitution of their identity, rather than conceding to external reflections and interactions. To illustrate this process, Rehak uses the example of PacMan, a character that is "recognizable as Pac-Man because of what [is] excluded from its form" (115). Like Pac-Man, we are incomplete, and therefore sympathize with his search for his "eternally missing slice" (115) because he mirrors our lack (124).

One of Rehak's most significant oversights, however, is that he fails to acknowledge that the player's relationship to the avatar is mediated through the developer. The avatar is not exclusively a specular image of the player, it has its own shape and form that is determined by the context and genre of the game. The Walking Dead is particularly limited in this regard, not only because it does not allow players to customize their avatar's attributes beyond variations in personality and disposition, but also because Lee exists both in the narrative context defined by the game's developers and within the expectations and conventions of the larger Walking Dead universe. Players' understanding of this universe affects how they behave in the game; for instance, players expect that when a survivor is bitten, that removing the infected limb will save them $-84 \%$ of players attempted this operation when given the choice in Telltale's game (The Walking Dead). ${ }^{7}$

\footnotetext{
${ }^{7}$ These statistics are subject to some fluctuation as new players complete each episode and others replay the series to explore alternate pathways.
} 


\section{Sing fond from the}

Fig. 4.1: screenshot from episode 2 of Telltale's The Walking Dead (2012)

The earlier iterations of The Walking Dead universe influence players' expectations about what is possible in Telltale's version. Although Telltale avoids repeating the same storylines by introducing new characters, the events and behaviours of characters are largely limited to the conventions of the series. One would expect that making difficult decisions about food resources and the fate of other characters would significantly alter Lee's character, and certainly, the player's decisions cause rifts between characters, but it is not possible, for instance, to villainize Lee. Consider the iconic scene where the survivors encounter the St. Johns, a family that runs a dairy farm, who appear to be very welcoming and generous. When the group is invited to stay for dinner, Lee discovers that the St. Johns intend to serve their friend, Mark, as the main course. When the St. Johns are confronted about the meal, the farmers provide very pragmatic and utilitarian reasons for their actions; they note that he was severely injured and that survivors should not be wasteful, that he would have died anyway, and that survivors should think about the living (The Walking Dead). In response, the player may 
choose from a number of dialogue options: the player can threaten the farmers or demand to be let go, but regardless, all responses lead to the same outcome, and the group is imprisoned. Players are not, however, given the opportunity to agree with the utilitarian approach of the farmers. This example highlights the limitations that are placed on the player's agency and the kinds of characters that can exist in this universe; as a result, the game forces a very specific kind of ethics.

This scene with the St. Johns also provides insight into how transmediation alters our experience of an event across different media. Both the comic and television series include a scene that parallels the one described; however, rather than utilitarian farmers, the cannibals in these static media are referred to as the Hunters, who, ironically, are quite poor hunters who have resorted to hunting humans. In both the comic and tv series, one person strays from the group and is caught by the Hunters: Dale, from the comic (Kirkman "Fear the Hunters") and Bob, from the television series ("Four Walls and a Roof'). In both instances, the Hunters keep the character alive after they have partially consumed them, and attempt to rationalize and explain their actions to their victim. Initially, the character appears to be upset, but then bursts into laughter, telling the Hunters that they had recently been bitten and that they are "tainted meat" (Kirkman). The characters were easy for the hunters to capture because they had intentionally left the safety of Rick's group to protect the other members. The readers and viewers, at first shocked at what has happened to their beloved characters, are given a kind of retribution when it is revealed that the characters were infected.

In Telltale's adaption of this event, however, this scene of retribution is absent, since Mark was not infected. In a certain way, this makes the scene more horrifying, as it 
lacks the same justice that is delivered in the comic and television series. However, unlike other media that have to give retribution to its viewers, the game enables players to choose revenge. When escaping from the farm, players are given the choice to kill or spare Andy, one of the St. John brothers. In the decision summary of episode two, the "revenge category" reveals that $82 \%$ of players chose not to kill both of the St. John brothers (see figure 4.1). Surprisingly, most players chose not to get revenge on both brothers, which conflicts with the comic and television series where revenge is delivered in full.

Unfortunately, any conclusions about these results can only be speculative. Nonetheless, these one-sided decision moments have been a concern for Telltale through the development of the series. After the release of the first episode of The Walking Dead, Telltale discovered an issue where $75 \%$ of players chose to save Carley over Doug in a life-or-death situation ("Episode 1 Stats Trailer"). As Telltale outlined in their talk at the 2015 Game Developers Conference, they had hoped that choosing between the two characters would be a difficult decision for players, and after examining the relationshipbuilding moments between the player and these characters, Telltale attributed the results to players having more opportunities to interact with Carley. This assumption, however, may not fully capture the motivation behind the player's decision; some players, for example, may have made the pragmatic choice to save Carley because she seemed more competent, or because she had a gun. Telltale states that they attempt to avoid one-sided situations by creating dynamic scenarios and not situations like "[p]unch the guy in the face or not" (Bissell, "When Story Is the Gameplay"), which asks the player for permission, rather than serving to establish compelling decision-making moments. 
Telltale emphasizes that although players may make poor decisions, the game does not make players feel as though they have made a wrong decision.

Despite Telltale's efforts, however, the statistics at the end of each episode reveal that a number of decisions were frequently one-sided. It is unclear if these results are due to a failure in the design of Telltale's decision making system, or a consequence of the influence of the larger Walking Dead universe on players (and even developers).

Although transmedia narratives introduce an extra dimension of complexity when designing decision-based games, such one-sided outcomes have also plagued developers of games with moral choices like Fable and Mass Effect. As Amanda Lange examines in her article "You're Just Gonna Be Nice," approximately 5\% of players choose to play evil in morality games (5) and, of those who would play a game more then once, about half indicated that they would play evil on a second playthrough (6). Although she warns that these results "should be interpreted cautiously" (3), her results strongly suggest that players' decisions lean toward good or neutral options. These kinds of results may have important consequences for developers who invest significant time and capital into developing large segments of the game that will not be seen by the majority of players. If players are not interested in choosing particular paths, developers may choose to avoid branching paths in favour of more ambiguous game outcomes, as seen throughout Telltale's games.

\subsection{False Choices and Empty Gestures}

While not all of the major decisions in The Walking Dead are game-altering, they are intended to stand out as important events in the game's narrative. Many of these 
choices are false, as Smethurst and Craps discuss in their paper "Playing with Trauma: Interreactivity, Empathy, and Complicity in The Walking Dead Video Game." Citing Mawhorter et al., they describe false choices as those "where all of the different options lead to the same outcome.... False choices can be used to create the illusion of a richly branching story without spending the resources necessary to do so" (qtd. in Smethhurst and Craps 283). For example, early in the first episode, Lee's group finds itself in a moment of crisis, and Lee must choose to save Kenny's young boy, Duck, or Hershel's adult son, Shawn. Regardless of what players choose, the outcome of the event is the same. Smethurst and Craps argue that the false perception of choice is equally important as the real events of the story. Like the concept of the "disnarrated" discussed in Chapter 2 , false choices bring complexity to the narrative through simplicity. These situations work well in the Walking Dead because it is difficult for the player to play through all of the possible scenarios to see if and how each decision affects the game. False choices create an illusory kind of freedom, whereby players believe that they are free to choose and can imagine the results of choices not taken. More specifically, Smethurst and Craps argue that The Walking Dead "manages to convince players that they have a real hand in determining how the story plays out" (283) and that this player-avatar connection has important consequences for feeling empathetic and complicit throughout the narrative. Reviewers who have played the game more than once, however, often express disappointment, as the illusion of choice is broken when they discover that the choices that they agonized over did not have real consequences. ${ }^{8}$

Similar to the false choice, Slavoj Žižek categorizes another kind of choice that he

${ }^{8}$ See store.steampowered.com/app/207610/ 
refers to as an "empty gesture" (Žižek, How to Read Lacan 12-13). In a certain way, an empty gesture is indistinguishable from a false choice in that one is faced with a situation in which the ability to choose is irrelevant. However, an empty gesture presents a choice where one option is implied to be the correct and only choice. As Žižek states, it is an offer that is intended to be rejected: "[t]his paradox of willing (choosing freely) what is in any case compulsory, of pretending (maintain the appearance) that there is a free choice although effectively there isn't one, is strictly co-dependent with the notion of an empty symbolic gesture, a gesture — an offer - that is meant to be rejected" (Žižek 12-13). Žižek offers the example of a competition between two friends for a job promotion. When one wins the promotion, they should, as a kind gesture, say that the other should have received the promotion, and in turn, the friend should decline and say that the other deserved it more. Žižek imagines that if one accepts the friend's offer of the promotion, that the situation would be "properly catastrophic" (13) and would cause "the disintegration of the semblance (of freedom) that pertains to social order, which equals the disintegration of the social substance itself, the dissolution of the social link" (13). In other words, by accepting the empty gesture, one draws attention to the very fact that the offer, although well-intentioned, is disingenuous, and, therefore, that freedom and choice are illusory.

While Telltale has to be careful about the kinds of choices that it offers players (due to content limitations), The Walking Dead makes use of empty gestures to make the player feel culpable for their actions. By evoking the compulsion to act in accordance with the social order, the game presents situations where a choice is given, but the game strongly indicates which choice is the correct one. Returning to the example of the St. 
Johns, an empty gesture occurs when the player is given the option to murder Andy, one of the St. John brothers, or spare his life and walk away: $82 \%$ of players chose to spare Andy (see figure 4.1). In this moment, while the player fights with Andy, one of the members of your group (which one is dependent on decisions made throughout the game) tries to dissuade the player from fighting and yells out "Lee that's enough. He's had enough" (The Walking Dead). In an attempt to preserve his dignity, despite the obviously one-sided fight, Andy taunts the player and tells Lee to "come back and fight like a man" (The Walking Dead), and Lee yells that it is over and begins to walk away. In case this dialogue was not enough to convince the player that they should spare Andy's life, the camera turns around on the player and shows Lee, whose facial expression reveals pity for Andy (see figure 4.2). Although the player can choose to kill Andy, doing so results in the group's disapproval and disappointment. It is clear that Andy has lost and has given up.

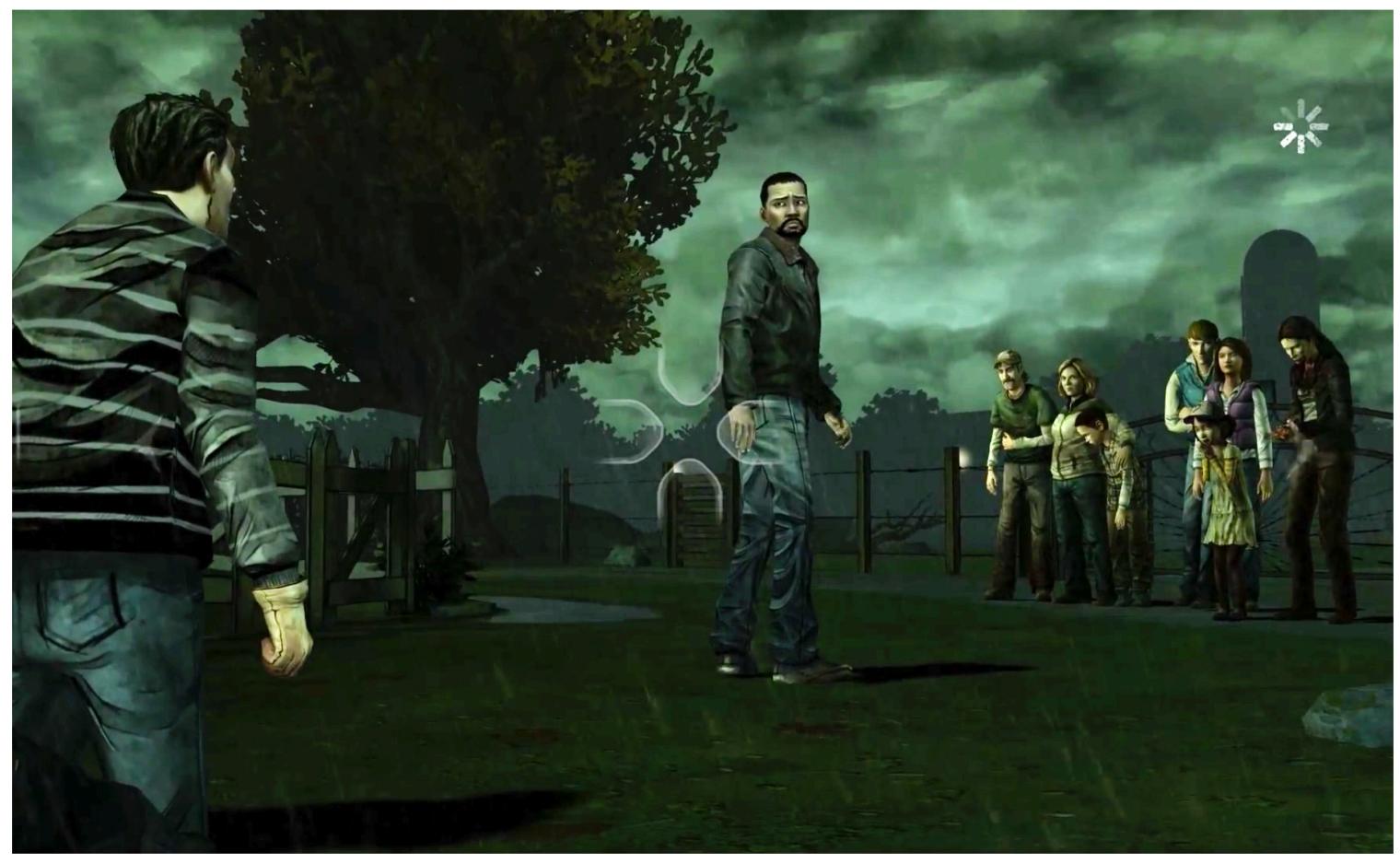


Fig. 4.2: screenshot from episode 2 of Telltale's The Walking Dead (2012)

Analyzing the scene in detail reveals how the game pressures players into making what is ostensibly not a genuine choice — both in the sense that this action does not have any consequences on the narrative, and in that it leads to the disintegration of what little is left of the social order. As Smethhurst and Craps explain, these close-ups of characters' faces have a strong effect on players:

According to theories about mirror neurons, when one witnesses the face of someone caught in an emotional state such as joy, shock, or suspicion, one subconsciously emulates that emotion, sometimes even mimicking the expression in order to feel it more strongly. This is one of the ways in which the game represents trauma: by showing us its impact on the faces of characters who are suffering through the shock of losing loved ones in harrowing situations, and the numbing and disbelief brought on by the complete dissolution of society as they know it. (284)

Facial expressions are a key aspect of Telltale's approach to storytelling in games and, although difficult to measure, there may be a link between players' responses to these expressions, their familiarity with the heroic narrative of The Walking Dead, and their tendency to avoid choosing evil paths. As Lange's study reveals, most players are already reluctant to act in discordance with their assumptions about how one should act in the social order. 


\subsection{Agency and the Player-Avatar Relationship}

This analysis of player agency and the commercial context of The Walking Dead upsets Rehak's claims about the avatar as a reflection of oneself, insofar as this reflection will not represent players unless their ideal-ego resembles Rick Grimes. If so, then the agency that the player believes that they exert over the game is merely a reflection of the kinds of outcomes and experiences expected in The Walking Dead universe, rather than an authentic reflection of players and their ideologies. In other words, should the player hold contrarian views, they will be displeased with the choices offered.

Yet, precisely because players are not meant to play as themselves, the game forces players to experience the world from Lee's perspective. In so doing, The Walking Dead offers a rare, positive example of the representation of a marginalized group in games. In Gaming at the Edge (2014), Adrienne Shaw examines how and why representation matters to players by conducting a series of interviews with people typically excluded from games based on race, gender, and sexuality. Her research reveals, contrary to expectations, that representation did not matter to her participants in the ways that one would expect; her interviewee's claim that "'He could be a bunny rabbit for all I care!' reveals the ways character identities can be largely irrelevant to players and to the game action itself" (131). This statement, however, does not imply that representation is not important; instead, it is precisely because players tend not to closely identify with characters that developers should be free to offer a diverse representations in games without rebuke. Furthermore, as her participants noted, although they did not identify with game characters, they thought that the dominant group needed to see accurate representations of people that are unlike them and "that more vulnerable people, like 
children or those who were marginalized, needed to see representations of people 'like them"” (197). As Shaw argues, "it is not game play that stands to benefit from, or even be dramatically transformed by, more diverse representations but rather culture more broadly" (143). These kinds of non-optional experiences resist player-centric trends and allow developers to create narratively complex characters that benefit culture in the way that Shaw envisions.

Unlike Rehak's metaphor of the player-avatar relationship as "a cat chasing its tail" (119), this analysis of The Walking Dead offers a more nuanced view of the complex connection that players have with their avatars that includes not only its commercial context as part of a larger franchise, but also the social order and conventions that sustain our everyday interactions. Although many of the pathways imagined by players are closed off through false choices and empty gestures, it is still possible for Lee to develop within a range of dispositions, but only those possible for protagonists within the accepted canon. Similarly, the game forces players to act in ethical ways that are congruous with the franchise's own take on human rights (i.e, it subscribes to a kind of Kantian ethics rather than utilitarianism). Despite the numerous statistical results that were highly similar among players, the final episode reveals that players' decisions have an effect on the dynamics of Lee's group, as demonstrated by those who were willing to help Lee when Clementine goes missing.

For transmedia works in particular, the player's decisions are always-already in dialogue with the larger narrative world of the series, and therefore rather than simply reacting to game events as oneself, the player's response is informed by existing knowledge of the franchise. The consideration of these sociocultural aspects establishes a 
more comprehensive theory of the player-avatar relationship that challenges earlier theories of agency that focused too closely on the player. The Walking Dead provides a crucial insight into the complexities of the player's relationship to the avatars and reveals, contrary to BioShock, that agency is possible by shifting attention away from plot to developing your character. While The Walking Dead's linear narrative structure may not ultimately diverge significantly from that offered by the BioShock series, it demonstrates an effective strategy for making player choices meaningful without compromising the length of the game or creating a structure that is too sprawling and complex to produce. Although the player does not get to determine the events of the narrative, they do have the ability to decide how they will respond to that world and who they will become. Despite the success of Telltale's model, the industry seeks to satisfy the player's demand for more input, more ways to change the world and the stories that it produces. The following chapter returns to this desire for a fully adaptive narrative to consider the implications of explicitly manifesting this desire by examining Benjamin Rivers' Home (2008), a game that, while not fully customizable, attempts to deliver a narrative that is tailored to the player's desired outcome. 


\section{Section III: Enjoyment}

\section{Chapter Five: Players as Authors}

\subsection{Narrating Play: Home}

In 2012, Benjamin Rivers' released Home, a game that he describes as a "unique horror adventure game" (homehorror.com). Home is unique not in its approach to the horror genre, but in the way that it constructs the player's relationship to its narrative; as Rivers' writes, "as you play the game, it changes — subtly, almost imperceptibly — to reflect your perspective. It's a horror game unlike any other, and as you'll discover, its truths are entirely subjective" (Rivers). The game begins with a conventional horror mystery: the unnamed character wakes up in a strange house where he finds a dead body. Uncertain how he has gotten there and whether the murderer is nearby, he is determined to escape to return home to his wife, Rachel. The game's narrative is largely linear, and the player's movement is similarly limited to left and right directions in its 2D space. As players progress through the game, they must try to piece together what has happened. The game, however, is not fraught with the usual difficulties that one would expect in a horror game: there are no challenging puzzles, health meters, or enemies. Exploring the game world reveals opportunities for players to pick up objects (a knife, a gun, a lost wallet), but these objects are never used in the game. The decisions that players make about how they interact with their environment (e.g., whether they search desk drawers or pick up objects) does not create branching narratives, but rather the game's narration changes to match the players' assumptions about their situation. Whether players choose to take or leave a gun offers insight into their perception of the events: leaving the gun might reveal a fear of incriminating oneself, whereas taking it might suggest that the 
player believes the character is the murderer. As the player makes more choices, the narration fine tunes its prediction of players' assumptions and more effectively narrates play.

This notion that narration and play can co-exist, however, is one of the points of contention in the ludology vs. narratology debate. Although this project has moved beyond this debate, the repressed content returns yet again. This particular dispute about time and narration, however, is worth examining in more detail to unpack the role of agency in narrative. In his contribution to the narratology vs. ludology debate, Jesper Juul argues that the two are incompatible; as he writes,

it is impossible to influence something that has already happened. This means that you cannot have interactivity and narration at the same time. And this means in practice that games almost never perform basic narrative operations like flashback and flash forward. Games are almost always chronological. ("Games Telling Stories")

Although games have become more cinematic since the publication of his article, for Juul, the conflict between narrative and games is a temporal problem related to agency: if a narrative is the retelling of events, and the events of the game occur in the present moment (as the player interacts with the game), the two, therefore, cannot happen at the same time. For developers, however, this barrier was more of a historical technical challenge than a constraint inherent to the medium. In addition to flashbacks and flash forwards, a number of games have been released in recent years that combine agency and narration. While Home is one example, others include Galactic Cafe's The Stanley Parable (2013) and Supergiant's Bastion (2011). Cut-scenes aside, narration in games is 
difficult because it requires the game to respond in real time to players' actions. The problem of narration in games is best understood by examining our own day-to-day relationship with narrative.

In "Life in Quest of Narrative," Paul Ricœur develops a theory of how we use narrative to make sense of our experiences. He addresses the supposed disjunction between life and narrative put forth by unnamed commentators who, he states, argue that "stories are recounted and not lived; life is lived and not recounted" (20). This view echoes Juul's claims about incompatibility between narration and interaction. Ricœur, however, rejects such assertions about narrative and life by examining the act of reading, and explains that while stories are recounted, they are also "lived in the mode of the imaginary" (27); in other words, the reader is transported to the story world and experiences its events as the present. As Richard Gerrig writes, "great artistry might facilitate the journey, but the only a priori requirement for a means of transportation is that it serve as an invitation to the traveler to abandon the here and now" (13). The invocation of a place — Texas in Gerrig's example — transports the reader to that narrative world (4). In a study, Melanie Green found that readers who felt transported were more likely to think "that the story was more like real life. . . that the situations were realistic and that the characters acted like real people would have acted" (262).

In turn, Ricœur considers how, in an analogous way, lives are also recounted in that narrative is an essential part of cognition. Citing cognitive scientists, Marie-Laure Ryan similarly argues, that "most if not all memories are indeed stored in the form of stories. I am not saying that life 'is' a narrative, but it can in certain circumstances 
suggest a quality that we may call "narrativity", (Avatars of Story 10). Ricœur builds on theories from cultural anthropology to argue that if actions can be recounted at all, it is because actions are "already articulated in signs, rules, and norms" (28); our actions are always symbolically mediated (28), and it is through this process of mediation that "we attempt to obtain a narrative understanding of ourselves" (33). Without narrative, life would be experienced as isolated stimuli and would lack coherence. Narrative provides a means of organizing experience, and by telling stories about these experiences, we come to understand the events of our lives (32). To illustrate, Ricœur offers the examples of a psychoanalyst and a judge, whose roles are to untangle and make sense of a series of events, thereby revealing the unconscious or solving the crime. In an analogous way, we are always engaged in these same processes as we untangle and make sense of our actions and experiences. Ricœur's critique of this disconnection between life and narrative reveals that interactivity and narration are not incompatible, but are both essential to meaningful experiences.

\subsection{A Psychoanalytic Approach to Games}

It is in this interstitial space between action and narration that a psychoanalytic approach to games becomes all the more relevant. Psychoanalytic approaches to narrative are not, as many would believe, concerned with analyzing characters. Although such approaches are popular in literary and film studies, these entertaining analyses of the unconscious motivations of characters are misapplications of psychoanalytic theory. Monika Fludernik provides an example of such a problematic application of psychoanalysis in An Introduction to Narratology; as she writes, 
I want to take a look at that part of the human mind which, since Freud, has been termed "the subconscious' ${ }^{9}$ and which it is not easy to accommodate in a discussion of the representation of thought. There is general agreement that what is subconscious cannot be verbalized although, according to Freud, it does include verbal elements. . . In point of fact, representing the subconscious (or unconscious) seems to be beyond the scope of narrative discourse. On the other hand, we do find a great many psychoanalytic interpretations of narrative texts, dealing with the unrecognized and unacknowledged wishes, impulses and obsessions of narrators and characters. (Fludernik 85-6)

Fludernik's suggestion that psychoanalytic theory be applied to characters and narrators runs counter to a methodology intended for clinical practice. While it may be entertaining to speculate about the Oedipal struggles of characters, as Mieke Bal states, "[c]haracters don't have an unconscious, only people do. Psychoanalytic criticism does not, or should not, consist of diagnosing characters but of understanding how texts affectively address the reader on a level that comes close to unconscious preoccupations" (Introduction to Narratology 121). Similarly, one must also not employ psychoanalytic theories to speculate about authors, as this approach reproduces problematic conflations of authors and narrators.

Instead, psychoanalysis should be taken as a practice that exists in a liminal space between writing and reading, between expression and experience. Psychoanalysis and

\footnotetext{
${ }^{9}$ It is imperative to note that the term "subconscious" was never used by Freud and is meaningless in Freudian and Lacanian discourse. The most similar structural counterpart would be the preconscious, which designates that which can easily be brought to consciousness (or things we know, but are not knowing right now), and which does not correspond to the unconscious (things that we do not know that we know).
} 
game studies have much in common: they are both discourses and approaches that are designed to attend to finicky objects and subjects that are complicated narratives on the surface and algorithms and code beneath. The player occupies a middle position between "writing and reading": they are simultaneously engaged in the game world and subject to it. A psychoanalytic perspective must not analyze the game world alone (its plot and characters), but instead should be player-centric (or, more broadly, examine games as a representation of the world). As Lorens Holm writes, "[p]sychoanalysis is not interested in how the subject sees the world, but in how the subject represents the world by word and image, to itself and to others, most notably, the analyst" (12). Narrative has a central role in this process of representation. In his critique of the opposition between life and narrative, Ricœur elaborates on the importance of narrative to life: "by means of the imaginative variations of our own ego ... we attempt to obtain a narrative understanding of ourselves, the only kind that escapes the apparent choice between sheer change and absolute identity. Between the two lies narrative identity" (33). It is in this liminal space that Rivers' game Home crafts its story: between its own scripted narrative and players' interaction with its system.

\subsection{Alterbiography}

In games, narrative identity does not strictly consist of the player's actions and narrative; it is located at the intersection of several points of interaction, including the player's own narrative identity. In In-Game: From Immersion to Incorporation (2011), Gordon Calleja proposes the term "alterbiography" to describe the multiplicity of the player's interaction with game narratives that is analogous to Ricœur's narrative identity. 
Calleja's approach to player experience is finely balanced between the ludological and narratological dimensions; it leans neither too heavily on experience nor narrative. Likewise responding to the criticisms presented by ludologists, Calleja critiques the narrow view of narrative employed by Juul and others to formulate his theory of "incorporation," which describes the way that the "player is not merely transported to a virtual world, but also incorporates that world into her own consciousness in a dual process"(173).

In his account of narrative, Calleja identifies two forms of narrative present in games: the scripted-narrative and alterbiography. The scripted narrative is the written story that occurs in the game world. Borrowing from BioShock's Ken Levine, Calleja further divides the scripted-narrative into both push and pull narrative elements. Push narrative includes segments of narrative that are forced on the player through cut scenes and monologues, while pull narrative are bits of story that must be discovered by the player (usually in the form of hidden letters, videos, and audio clips) that draw the player further into the narrative world. In Home, the push narrative is text dialogue that introduces its setting, while the pull narrative consists of the curious objects that are not required to be found to complete the game, such as the video tape. These types of narrative elements are used to craft a specific kind of experience for the player. 


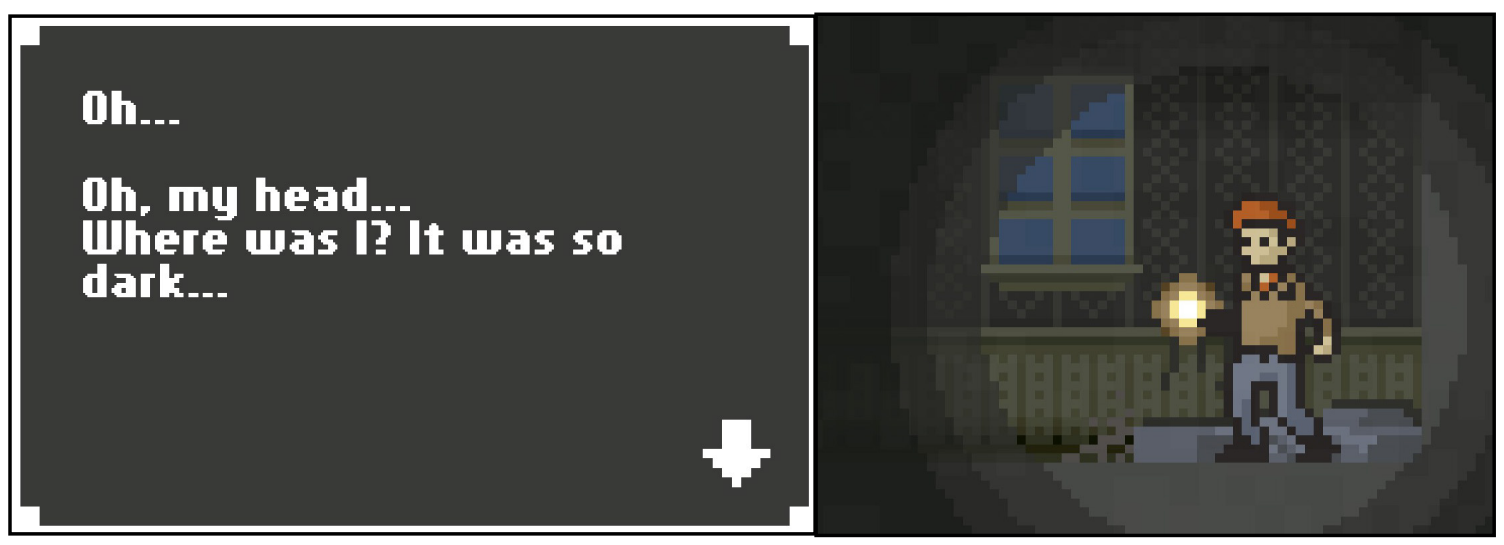

Fig. 5.1: screenshots from the opening scene of Benjamin Rivers' Home (2012)

Calleja's alterbiography, however, is not supplied by the developer; it is produced by the player in conjunction with the game's narrative, and it is not mutually exclusive to the scripted narrative. As Calleja writes, the alterbiography is the "ongoing narrative generated during interaction with a game environment, it is neither solely a formal property of the game, nor a property of the player's free-roaming imagination" (124). The alterbiography is informed by both the scripted narrative and the players own subjective interpretation of events and experiences both in and out of the game. Calleja illustrates this concept through Rockstar's Grand Theft Auto series, where a player is preparing his character for a date when the character bumps into a non-playable character and unnecessarily starts a fight, which quickly draws the attention of the police. The player's subjective interpretation of the events is that the character is tired and irritable after failing the last few missions, which led to his confrontation with the unsuspecting nonplayable character. In truth, it is the player's own disposition and experiences that informs the narrative constructed by the player about the character; challenging Juul, Calleja writes that "interaction generates, rather than excludes, story" (115). Calleja's account of the narrative potential of Grand Theft Auto is also opposed to Marie-Laure 
Ryan's argument that Grand Theft Auto is a "narrativized video game," rather than a "playable story" (Narrative as Virtual Reality 2 234), which has most in common with the "walking simulator" genre. In Narrative as Virtual Reality 2, Ryan argues that in "narrative video games" the narrative is used to sustain the player's activity, but is not central to it; as she writes, "in the intensity of the action, players may forget whether they are terrorists or counterterrorists, space aliens or defenders of the earth; in a narrative game, the player plays to win, to beat the game, and story is mostly a lure into the gameworld" (234-35). For Calleja, however, narrative games and playable stories are not quite so far apart. The narrative of Grand Theft Auto offers something more than a justification for the player's activity, as it is co-produced through the game's scripted narrative and the player's alterbiography.

What makes Home a unique game is that it incorporates the player's alterbiography and feeds it back to the player as the game's scripted narrative. As the player makes choices throughout the game, the context in which these decisions are made is ambiguous. While searching the house, the player may become concerned that they will incriminate themselves, and after deciding not to search through a desk, the narrator states "I really shouldn't have been going through this stuff, and getting my fingerprints everywhere" (Home). The player may also suspect that the murderer will soon return, or that their character is the murderer. The assumptions that the player makes about their situation informs the outcome of the narrative based on how they interact with the environment. As an example, consider the scene early in the game when the player finds a weapon: "A work table, covered in papers, dirt and... a handgun. I hated guns. I didn't take it, did I?" (Home). The user may choose to take the gun or leave it behind. If the 
player chooses to take the gun, the narrative reads "I tucked the small handgun into my pocket. Its weight was somehow reassuring," or if the player decides to leave the gun behind, the dialogue reads: "I didn't even want to touch the thing. Better I left it right where it was" (Home). Implicit in these responses is the player's suspicion about the character's role in this murder mystery, and as they make choices, the player's alterbiography runs parallel with the scripted narrative: the game manifests the alterbiography by narrating play.

Although it is clear as you play through the game that your decisions have an impact on its outcome, nowhere is this fact more explicit than in the final moments of the game, when the player finally arrives home and searches the house for the character's wife. When players enter the basement, they find something suspicious:

A filthy-looking pile of rags had been dumped in the corner. The stench of them was awful and made my eyes water. I was terrified to even touch the pile — to see what lay within. But I knew I had to; I had come this far. After all this searching, after all I had seen... When I looked within the rags, did I finally find my Rachel? (Home)

Players are given a choice: yes or no, they must decide what is found in the pile. Following their response, things in the house begin to change, "subtly, almost imperceptibly" (Rivers). Depending on choices made throughout the game, players may find that the books on the shelf, once shared between husband and wife, now belong only to your character; or perhaps the expensive television that the couple had argued about buying, is replaced with one that is more modest and aged. While some changes suggest that perhaps Rachel had never existed, others allude to your role in her disappearance. 
When the game ends, players are provided with a link to the game's website, where they can submit their own experiences and stories about what happened in the game. A brief survey of the site's "What Happened?" page reveals that although players responses revolve around a rather limited series of events, their experiences were quite varied: some players imagined complex affairs, cases of dissociative fugue states, serial killers, alcoholism, fantasies, and delusions as explanations of the game's events. Unlike other multilinear games, Home does not simply have multiple endings, but each ending is crafted by the player's alterbiography in conjunction with the game's scripted narrative.

Although Home has largely positive reviews, ironically, some players were disappointed that the game was too predictable, and those who replayed it were often disappointed when they uncovered its trick, and felt strangely cheated by a game designed to deliver what they expect. These criticisms, however, reveal a kind of Catch22 that developers face when creating player-centric games that must make agency meaningful, but not in a way that actually gives players what they want. This is not a criticism of Home, but rather as a general model, Home's narrative structure reveals the inherent paradox of desire: that fully realizing what one desires often incites revulsion as the adage goes, "Be careful what you wish for." While it is easy to imagine why we would be disappointed if narratives were always predictable and ended as expected, psychoanalysis provides crucial insight into the workings of desire and its relation to the player's interaction with the game; for instance, how do players enjoy games and why is agency crucial to this experience?

In Creative Writers and Day-Dreaming, Freud argues that as children we play and act out our fantasies and wishes, but as adults we are made to give up this enjoyment and 
to stop playing. Of course, as he says, we can never give up anything, "we can only exchange one thing for another" (1923). So instead of playing, Freud suggests that our enjoyment becomes mediated. At least part of the reason that we enjoy fictional narratives is that writers enable us to enjoy our own day-dreams and fantasies without reproach or shame. They mediate our desires, and re-present them to us in a more agreeable form. Children, for instance, often enjoy imagining that they are superheroes, but to do this as an adult is viewed as embarrassing. It is, however, completely acceptable to watch superhero movies. In an analogous way, games provide a fantasy space that offers a permissible and mediated experience of desire. Mediation is crucial to the experience of desire, as is revealed by examining Home's unique narrative mechanic. The paradox of fantasy is that it simultaneously constitutes and protects us from our desire. As Žižek explains, fantasy shields us from experiencing the anxiety of directly encountering the object of our desire: "it is not the lack of the object that gives rise to anxiety but, on the contrary, the danger of our getting too close to the object" (Looking Awry 9).

On the one hand, our relationship to desire is complex because it is insatiable: when we get what we want, rather than being fulfilled, our desire shifts to new objects; attaining the desired object entails the redistribution of the drive to a new object: the object of desire is "a lost object which must be continually refound" (Evans 205). On the other hand, directly encountering the object of desire produces at best displeasure and at worst horror. Part of what attracts people to games is that they offer agency without consequence. As Rehak notes, avatars allow players to live without the consequence of death (107), but the desire for agency goes beyond immortality; or, more radically, as a 
manifestation of the death drive, it is the inverse of immortality. At its heart, agency promises to free the subject from the Other's desire. In such a fantasy where one has complete agency over one's experiences, one would be untethered from the demands of the Other and free to do as one pleases. One's desire, however, is always tied to the Other's desire: as Evans writes, "what makes an object desirable is not any intrinsic quality of the thing in itself but simply that it is desired by another" (Evans 38). While our obligations and commitments may feel burdensome at times, the reality of total agency would create a world devoid of desire and surprise. For this reason, desire must be mediated.

This counterintuitive measure is necessary for the reason that desire itself must never be fully satisfied. The very nature of the cause of our desire (or objet petit a) is such that it incites both desire and revulsion. It is for this reason that the aim of desire is not to obtain its object, but rather to circle around it. As Žižek succinctly states, "we mistake for the searching and indecision proper to desire what is, in fact, the realization of desire. That is to say, the realization of desire does not consist in its being 'fulfilled,' 'fully satisfied,' it coincides rather with the reproduction of desire as such, with its circular movement" (Looking Awry 7). Thus, games not only serve as a means of approaching or staging our desire, but they simultaneously protect us from the abject Real of our desire. Rather than finding enjoyment in the search itself, one mistakenly pursues the object of desire with the belief that the object itself is necessary for enjoyment and that one would be able to obtain the object if only some constraint were removed.

It is precisely this deadlock of desire that is exploited in advertising, where one is subject to a kind of proof that others are capable of enjoying the desired object beyond 
the limits of one's own ability to enjoy. The fantasies that are staged in advertisements not only make it appear certain that obtaining the object of enjoyment is possible, but that one should feel guilty for not already possessing it. Fantasy serves an important means by which one is able to mediate the desired object: through fantasy one is able to approach the object while also keeping it at a distance. Contrary to its more conventional understanding, the term fantasy is not to be understood as a simple indulgent hallucination, but rather in the psychoanalytic sense:

fantasy is the very screen that separates desire from drive: it tells the story which allows the subject to (mis)perceive the void around which drive circulates as the primordial loss constitutive of desire. It other words, fantasy provides a rationale for the inherent deadlock of desire: it constructs the scene in which the jouissance we are deprived of is concentrated in the Other who stole it from us. (Žižek, Plague 32)

For instance, the narrative that new technologies will bring games closer to fulfilling the promise of the Aleph serves to simultaneous incite the desire for such an experience, while also explaining away the failure of games to fulfill this promise. Fantasy is the narrative that sustains the illusion that agency and free will exist. It provides a justification for the absence of complete control while maintaining its possibility. As a model for game development generally, Home reveals that relying on the alterbiography is not a trick that can be repeated. As much as players desire to exert agency over a game's world and narrative, they do not actually want to experience such a predictable world, and would inevitably be repulsed by such a game. Given the nature of desire, it is imperative to investigate how players enjoy games. 
The following chapter examines how the player's interaction with a game produces enjoyment though Lacan's clinical structures of perversion and neurosis to critically theorize how enjoyment emerges in particular game structures. This understanding of a game as both a world constructed by its developers as an absolutely determined game space, and yet also open to user experience and interpretation, entails a kind of cognitive dissonance. Taking Jonathan Blow's Braid (2008) as an exemplary work of proceduralism in practice and Namco's Katamari Damacy (2004) as its structural inverse, this chapter will address player enjoyment by examining games as complex and conflicting systems of rules, mechanics, and narrative. 


\section{Chapter Six: Perverse and Interpassive Gaming \\ (Or, The Pervert's Guide to Steam $)^{10}$}

\subsection{Enjoy!}

Our enjoyment of many games comes from their ludic elements and the reward of overcoming their difficult challenges. In The Grasshopper: Games, Life and Utopia, Bernard Suits proposes that the very definition of "playing a game is the voluntary attempt to overcome unnecessary obstacles" (41). This definition, however, no longer fully captures the range of experiences offered by contemporary digital games. In "Without a Goal: On Open and Expressive Games," Jesper Juul explores the expressive potential of goalless games. While games such as The Sims and Grand Theft Auto may have intended goals, they also offer an expansive game world in which players are free to disregard the larger objectives of the game to pursue their own aesthetic and ludic desires; for instance, Juul writes that players who customize their characters' homes in The Sims may purchase "not the optimal chair, but a beautiful chair" (192). Juul argues that these games are enjoyable because the expressive potential of what they offer encourages different playing styles, meaning that players are free to create and explore in their own way. Any account of player enjoyment must account for the variety of experiences possible in games.

In his article "On Video Games, Culture, and Therapy," Kourosh Dini (2012) addresses the fact that analysts now regularly encounter analysands who play video games, and argues that analysts must be prepared to address experiences and interactions

${ }^{10}$ An earlier version of this chapter was published as "Perverse and Interpassive Gaming: Enjoyment and Play in Gamespaces." Psychoanalysis, Culture \& Society, vol. 22, no. 1, 2017, pp. 106-113. 
with new media, or else they "risk alienation from a significant part of their patients' lives" (499). By understanding how players interact with video games, analysts will be better prepared to address how virtual spaces "harness or hinder various paths and aspects of development in analysis" (496). How one plays and enjoys games is essential to Dini's approach to understanding one's behaviour in day-to-day life. Through two case studies, Dini exemplifies how the analysand's relation to the avatar can mimic or replicate problematic, real-life behavioural patterns. This parallel between life and games is supported by Lacan's own thinking about reality, as André Nusselder states:

When I play a game, that is to say, when I represent myself as a player of a certain game, I am inescapably submitted to the rules that determine how the game should be played: how one should interact, and so forth. Lacan considers human reality, in its most fundamental form, to be a "game" also — as shown by his saying that the principle of reality is the principle of collective fantasy. (45) While Dini's work provides an important step for conceptualizing a practical and interdisciplinary approach to new media in analysis, it is insufficient insofar as it overlooks scholarly contributions to the field of game studies that would strengthen his observations. By incorporating the work of video game theorists, this chapter reassesses Dini's approach to games to address a relationship between player and game that goes beyond identification and the player-avatar relationship.

Although games appear to reproduce or transmediate the familiar narratives of other media (e.g., novels, film), our interaction with these works cannot be understood using the same methods; as Galloway explains, "[t]o play the game means to play the code of the game. To win means to know the system. And thus to interpret a game means 
to interpret its algorithm (to discover its parallel 'allegorithm')" (Gaming, 90-91). As Wark elaborates, "[t]he allegorithm by which the gamer relates to the algorithm produces a quite particular allegory by which gamer and algorithm together relate to gamespace" (030). While most players do not have access to the code of a game, they must intuit its algorithms in order to succeed. It is this level that is overlooked in accounts that emphasize the player's relationship to the avatar or character. For a complete understanding of the player-avatar relationship, analyses must consider both its allegorithmic and allegorical dimensions. A game's narrative (including aspects such as character depth and point-of-view) and how the player interacts with that world are central to the kind of relationship that the player forms with the avatar or character (e.g., empathetic, sympathetic, detached, or, most rarely, an identification with a character)(Papale 8).

Lacan makes an analogous criticism of psychoanalytic theories that fail to theorize the role of the symbolic: by neglecting the symbolic dimension, one "reduces the psychoanalytic encounter to an imaginary dual relationship" (Evans 49). This criticism is fundamental to many of the psychoanalytic approaches to games that analyze the playeravatar relationship. By correcting this misstep, this study provides a more comprehensive framework to address the complexities of games as a medium, and the dynamic and problematic ways that players interact with games. For Lacan, the symbolic realm is linguistic, and both language and algorithms share qualities like grammar, syntax, and semantics. Rehak's attempt to analyze the player-avatar relationship through its ability to mirror our own split subjectivity similarly neglects to incorporate not only aspects central to game studies, such as narrative, genre, and mechanics, but also the relationship of the 
imaginary to the symbolic. Moving beyond such avatar-oriented approaches is necessary to address the implications of an industry that emphasizes agency, choices, and freedom. In part, jan jagodzinski's psychoanalytic analysis of violent games overcomes the limits of these avatar-oriented approaches, but, as the following section will demonstrate, his analysis becomes problematic in new ways.

In the first case study, Dini examines his analysand's decision to play as a solitary and independent character type in a massively multiplayer online game. The analysand's character selection, he notes, resembles and reinforces her own less socially-oriented behavioural patterns. To disrupt this cycle of repetition, Dini recommends that the analysand experiment with playing more supportive character types, such as a healer. This interpretation of the analysand's character choice suggests that Dini views the player-avatar relationship as a form of identification in a way that is similar to conceptions of the player-avatar relationship found in the work of Rehak (2003), discussed in Chapter 3, and jan jagodzinski (2006). It is rare that players identify with an avatar; more often, when given multiple avatar options, the player's selection is often made for strategic reasons (related to skill level or to balance team abilities), or to represent (and frequently misrepresent) oneself. Many women, for example, choose to play as male avatars in order to avoid harassment in multiplayer environments. Further still, many games offer very few, or even no, character choices, while others allow complete character customization.

While Dini was likely correct to encourage his analysand to engage more actively with other players in the game (given his description of the analysand's behaviour), it is important to recognize that the relationship between the player and the avatar is 
multifaceted, and is only one site of interaction in a game. Dini's approach to the playeravatar relationship, like Rehak's own analysis, is ambiguous about the conditions that produce the kind of relationship that it describes, as not all games construct avatar relationships in the same way. These analyses are reductive, and overlook the complexity and dynamics of the player's relationship to the avatar. Emphasizing the player's avatar risks limiting games to their narratological dimension, and consequently privilege the game's story over its mechanics, in contrast to the ludological approach, which argues that "the game is its rules" (Sicart, "Against Procedurality"). A more balanced approach that addresses the complexity of a game's system is essential to a comprehensive analysis of the player's experience. This perspective, however, entails a kind of cognitive dissonance: one must acknowledge that a game is simultaneously a highly constructed environment and also open to player interpretation.

One notable advocate of the ludological approach is Jonathan Blow, an independent game developer and the creator of Braid (2008). Blow's position on the discordance between ludology and narratology has very much influenced the development of his games; in an interview with Chris Dahlen, Blow states that "Braid isn't a subjective work of creativity: it's a system, meticulously designed to convey a meaning that really isn't up to broad interpretation. If you think the puzzles are too rigid, or you hate the text—maybe it's because you don't understand the point." Narratologists, however, challenge this notion of authorship and intention, as Mieke Bal writes, "not to deny authorship but to emancipate both author and reader from the stronghold of a misconceived interpretive authority" (16). It is for this reason that both perspectives must be incorporated in order to produce a more nuanced view of the player's experience; after 
all, games are meant to be played for fun and, if a game is foremost defined by the execution of its rules, where is the enjoyment in playing? As Eric Zimmerman notes,

There is a curious relationship between rules and play. In the classical sense of a game as a magic circle, rules are fixed, rigid, and closed. They are logical, rational, and scientific. Rules really do not seem like much fun at all. But when rules are taken on and adopted by players who enter the magic circle and agree to follow the rules, play happens. Play in many ways is the opposite of rules: as much as rules are closed and fixed, play is improvisational and uncertain. Yet in a game, these two opposites find a common home — gameplay paradoxically occurring only because of game rules. (26)

All games, of course, consist of rules, but the question of enjoyment is key to understanding the analysand's engagement with video games. To develop a more thorough approach to theorizing games in analysis, this chapter examines Braid and Katamari Damacy (2004) in relation to enjoyment through Lacan's clinical structures of perversion and neurosis, and explores Lacan's third structure, psychosis, through jan jadogzinski's analysis of the ethics of violent games.

\subsection{Perverse Enjoyment: Braid}

Braid, as an exemplary work of ludology in practice, serves as a particularly interesting example to explore in relation to enjoyment due to its restrictive game mechanics. Unusually, the game begins in the second world, where it introduces a conventional narrative: the player's character, Tim, is on a quest to rescue a princess who has been kidnapped. To complete the game, players are required to solve puzzles in order 
to collect puzzle pieces that unlock new levels. After collecting each of the pieces, the player is finally able to progress to the final (and also the first) level, where Tim confronts the "horrible and evil monster" (Braid) who kidnapped the princess. The solution to each puzzle requires the player to manipulate time, and each world introduces a new mechanic or rule, such as "reverse," "slow," or "transcend" time. Blow explains that the game's mechanics were inspired by his interest in quantum mechanics:

We don't know exactly where our current perception of time comes from, and why [time] seems to flow in one direction ... and we don't know why there didn't evolve a mechanism that can remember what happens tomorrow as well as what happens yesterday. [According to] the fundamental law of [quantum mechanics], there doesn't appear to be any reason why that's impossible. (Dahlen)

This statement demonstrates a certain logic that not only reveals much about how the game's narrative and mechanics inform one another, but also why Blow believes that his game, like its puzzles, has only one correct interpretation. Blow's understanding of time makes the mechanics of his game all the more interesting. While initially the manipulation of time in each world appears to give the player the freedom to correct missteps, or exert control over the game's interactive objects, ultimately these mechanics ensure that the player remains on the game's predetermined path. Thanks to the game's rewind function, moreover, there is no possibility of failure; consequently, all playthroughs of the game would appear identical, as players must execute precise actions to complete its puzzles. Failure to produce precise actions results not only in postponing one's progress, but often in frustration. 
After players have collected all of the game's pieces, they are finally able to save the princess. In the final challenge of the game, Tim and the princess appear to work together to escape her captor. Upon reaching the conclusion of the level, the princess returns to her castle, leaving Tim alone outside. The player quickly discovers that the only available action to progress the game is to rewind time. This act reverses the scene of their escape, which unexpectedly reveals that, in fact, it was Tim (the true "horrible and evil monster") from whom the princess was escaping. This event brings the game full circle, with the princess once again taken from Tim because he had made a mistake. The circular conclusion of the game explores the question of what time (and life) would look like if we could remember the future, and "could remove the damage [of our mistakes] but still be wiser for the experience" (Braid).

Braid's game design unabashedly conforms to the ludological model that produces games that are meant to be worked through rather than played. This vision of games aligns with Slavoj Žižek’s assertion concerning products that have had their damaging properties removed: "coffee without caffeine, cream without fat, beer without alcohol" (How to Read 38), such that there are now "games without play"- that is, "play" in the sense that Katie Salen and Eric Zimmerman define it, as "free movement within a more rigid structure" (304). The enjoyment of a game like Braid is therefore derived from working through its rules and the imposition of its structure, rather than playful interaction with the game world. This form of enjoyment is analogous to Lacan's structure of perversion, as Žižek elaborates in "The Cyberspace Real”:

$[P]$ erversion can be seen as a defense against the Real of death and sexuality, against the threat of mortality ... a universe in which, as in cartoons, a human 
being can survive any catastrophe. ... As such, the pervert's universe is the universe of pure symbolic order ... [Cyberspace is] constrained only by its selfimposed rules. ... In this comic universe, as in a perverse ritual, the same gestures and scenes are endlessly repeated, without any final closure.

Contrary to the more conventional understanding of the pervert as the one whose enjoyment is derived from transgressing the Law, the Lacanian pervert designates a subject whose relation to enjoyment depends upon rules and laws; as Žižek states, the pervert's "phantasmic narrative does not stage the suspension-transgression of the Law, but is the very act of its installation" (Plague, 14). In a world in which one cannot die and scenes are endlessly repeated, one depends upon the imposed structure and rules to give meaning to chaos. It is for this reason that play is absent from Braid; or rather, that play is instead turned into work.

The conflation of work and play is prevalent in contemporary society. Fredric Jameson (1991), for instance, writes that play may "no longer mean very much . . . in a situation in which leisure is as commodified as work, free time and vacations as organized and planified as a day in the office" (147). Galloway (2012) accurately characterizes contemporary life as dominated by micro-labour, writing that workspaces "have ballooned outward into daily life to such a high degree that labor is constantly performed by phone in the car, on email walking down the street, or at home after putting the children to bed" (Interface Effect 135). No doubt this is a practice that many recognize and have largely accepted as a practice that is simply a part of one's job and daily activities. That our leisure time has become infused with work is only further complicated by Wark's (2007) claim that work is also being transformed into play; in 
other words, work has become gamified as mundane tasks are made into competitive challenges to motivate bored and listless employees. Rewards and achievements are offered indiscriminately for fulfilling the demands of work. As children we learn to "play the game:" make more friends, participate in sports, and win more gold stars, the purpose of which, Wark writes, "is to build character" (011).

This conflation of work and play is directly related to Todd McGowan's assertion in The End of Dissatisfaction? (2012) that society is no longer structured around prohibition, but has been transformed into a society of commanded enjoyment (5). Rather than prohibiting enjoyment in order to ensure that members of society are productive, society now demands that its subjects enjoy. McGowan offers the particularly apt example of President George W. Bush's speech following the attack on the World Trade Centre that demanded that citizens spend money and go to Disney World "[i]n order to resist the terrorist attempt to derail the American economy" (36). Such a statement is explicit in this pronouncement that play has not only transformed into work, but has become one's patriotic duty. Needless to say, this command to enjoy, as McGowan writes, "merely produces a sense of obligation to enjoy oneself; it does not produce enjoyment" (37). In Braid, we find the inverse: one must find satisfaction in one's labour in a game played for leisure. Following the economic crisis of 2008, some commentators have blamed the enjoyment of games for the decline in men's participation in the workforce (i.e., in lieu of searching for employment, men were choosing to remain in virtual worlds) (Morris 2017). Others, however, have argued that games offer a source of pride and productivity that counters the emotional and mental side effects of unemployment and underemployment (Avent 2017). Braid provides a clear example of a 
game that supports this latter position, as the satisfaction that one derives from completing its puzzles is comparable to that which one would hope to gain from one's career.

\subsection{Neurotic Enjoyment: Katamari Damacy}

A comparable injunction to the command to enjoy is found in Namco's Katamari Damacy. The game begins with the King of All Cosmos demanding that his son, the Prince, rectify a mistake that the King had made the prior evening in accidentally destroying all of the stars in the galaxy. In order to restore the stars, the Prince must use a katamari ball to roll up any and all things on Earth, and then bring the completed katamari to the King to be turned into a new star. Though initially this appears to be a simple task, the King is incredibly particular about the quality of the katamaris that will be turned into stars. Invariably, when the player offers a completed katamari to the King to be inspected, he will declare his great disappointment. Even when the player exceeds the required size for a given task, the King complains: "If it were Ours, We'd make it much bigger" (Katamari Damacy). Wark (2007) argues that Katamari Damacy takes work and turns it into a game by scoring and ranking each of the player's katamaris. Indeed, the challenge of receiving even the most modest compliment from the King is so difficult that Wark appropriately compares the game to the myth of Sisyphus (076). This myth was also adapted by Pippin Barr (2012) into an even more frustrating mini-game in his Let's Play: Ancient Greek Punishment collection.

Like the neurotic, every attempt by the player to please the King (whom one could effortlessly analogize to the superego) is met with inadequacy, failure, and guilt. In 
such hopeless circumstances, the neurotic's response to the impossible demand is to attempt to postpone his or her encounter with enjoyment in order to maintain belief in its possibility. In other words, to preserve the fantasy that enjoyment is possible, the neurotic will engage in ceaseless activity in order to render the Other passive. Žižek (2003) refers to this act of deferral as "interpassivity." If, when play is turned into work (as demonstrated through Braid), the enjoyment of a game is structured like that of perversion $(\mathrm{a} \diamond \$)$, when work is turned into play, enjoyment is structured like neurosis $(\$ \triangleright$ a). Unlike Braid, in Katamari Damacy the player is able to explore the game world freely within the limits of whether one chooses to roll the katamari to a certain size as quickly as one can, or to make it as large as one can within a set time. Otherwise, the player may set their own goals irrespective of those dictated by the game. For instance, it is not uncommon for a player to become fixated on a particularly elusive object in the game world, or set a personal goal unrelated to the King's impossible demands. This kind of metagame or play that occurs outside of the game's dictated objectives is referred to by Wark as "trifling” (040). The player's activity is not invested in the aims of the game; that is, the player cares little about restoring the stars to the universe (to "win" the game) and instead becomes absorbed in unlocking more space for exploration and chasing personal objectives. Enjoyment in Katamari Damacy thus comes through the deferral of the game's objectives, rather than reaching its conclusion.

The possibility of trifling, however, does not provide a truly liberating experience, in contrast to a ludological game like Braid. One is, of course, always confined and operating within a game's algorithms and rules (098); yet, nevertheless, the act of trifling produces a distinctly different form of enjoyment. In his second case study, Dini 
encounters an act similar to trifling when playing an unnamed simulation game with his analysand. As Dini (2012) explains, "it immediately became apparent that her methods of dealing with the game's inherent challenges were strikingly similar to the methods developed elsewhere in life: She cheated. In fact, she cheated a considerable amount" (303). For Dini, cheating appeared to be a way to make the game easier; however, cheating often has little to do with winning the game and everything to do with changing its rules and structure. Whereas Dini felt that the analysand was missing the entire game (303), it is equally plausible that the analysand was instead playing an entirely different game irrespective of the game's goals.

The insights of game studies into the dynamic of play and work produces a strikingly different interpretation of the player's interaction with game spaces than those revealed in Dini's case studies. Examining the player's relationship to enjoyment reveals a new perspective on games in clinical psychoanalysis that moves beyond the specular image of the avatar. While the avatar may mirror one's split subjectivity (as an imperfect reflection of the player), games do not operate exclusively at the level of the imaginary. Our enjoyment is linked not only to a game's narrative, but also to its structure, underlying mechanics, and the challenges that it offers. As already demonstrated, new media technologies must be analyzed as complex systems consisting of varied levels and sites of interaction. Approaches such as Robin Hunicke's et al.'s MDA framework have been influential for thinking about how the mechanics, dynamics, and aesthetics of a game work together to shape the player's experience. 


\subsection{Psychosis and Ethics: Violent Games}

In "Video Game Cybersubjects, the Ethics of Violence and Addiction" (2006), jagodzinski draws on Rehak's analysis of the avatar and Lacan's work on psychosis to analyze the ethics of violent games. jagodzinski's states that debates about the effects of violent games on players are divided between two sides: the popular "moral panic" view and the advocates of "edutainment." The moral panic view passionately argues that children must be protected "from being corrupted and psychologically damaged" (283) by the violence in games; whereas proponents of games as edutainment, such as Henry Jenkins and Jane McGonigal, argue that playing games enhances learning and provides children with technical skills for the future (283). Although jagodzinski has established a somewhat false opposition (as edutainment games may include, but are certainly not limited to violent games), part of what one can learn from games is how to address violence and aggression. jagodzinski explains that, "[g]iven that violence and aggression are ontological themes that define our humanness, the importance of video games are sold as ethical testing grounds, much like the classic fairy tales of Brothers Grimm, Andersen and Perrault" (284). As an example of this ethical position, jagodzinski cites The Sims designer, Will Wright, who argues that "games are the only medium that enables a player to experience guilt feelings over the actions of the created avatars" (284).

jagodzinski, however, challenges this myth of games as ethical testing grounds by considering instances of gameplay in which players maimed and murdered their created Sims characters. The violence and context of such games, he argues, reproduces the values of neoliberal capitalism. This view of The Sims is not shared by all. Contrary 
to jagodzinski, who views the horrors that The Sims characters experience as a reflection of its violent and demented players, Galloway argues that "The Sims ... instead of seizing on the totality of informatic control as a theme ... does the reverse, diving down into the banality of technology, the muted horrors of a life lived as an algorithm" (102). The avatar's actions are not simply an expression of the player's repressed aggressions toward the people in their life that they have manifest in the game, but rather this violence punctuates a social critique of society's control structures. As Galloway elaborates,

The Sims is a game that delivers its own political critique up front as part of the gameplay.... The boredom, the sterility, the uselessness, and the futility of contemporary life appear precisely through those things that represent them best: a middle-class suburban house, an Ikea catalog of personal possessions, crappy food and even less appetizing music, the same dozen mindless tasks over and over-how can one craft a better critique of contemporary life? (Gaming 103-4) By considering the symbolic and algorithmic dimension of the game, Galloway produces an alternate reading of the game's sometimes violent play. jagodzinski's analysis, however, is centred on the violence itself, rather than its intention. While violent imagery and actions may not cause players to be more violent, violence has a desensitizing effect that he argues has its own problematic consequences (287-88).

jagodzinski addresses the ethics of violent games through Lacan's well-known inversion of Dostoyevsky, which he misquotes: "If God does not exist everything is permitted" (qtd. in jagodzinski 285). The correct quotation appears in Seminar II: The Ego in Freud's Theory and in the Technique of Psychoanalysis as, "If God doesn't exist, 
then nothing at all is permitted any longer" (128). Through this misquotation, jagodzinski aims to attribute the popularity and permissive attitude toward violent games and other media to a shift in the social order from prohibition to permission, as discussed earlier in relation to McGowan, Wark, and Galloway. McGowan argues that the injunctions of prohibition sought to limit enjoyment to ensure that members of society remained productive. For Lacan, it is precisely the Father of Prohibition who makes enjoyment possible through small transgressions of the law. jagodzinski is right to claim that the Father of Prohibition has disappeared and that a new permissive attitude has been adopted; however, God is not dead, but has been replaced by the postmodern Father of Enjoyment. The contemporary social order is instead controlled by the injunction to "Enjoy!" Instead of producing excess enjoyment through the permissive attitude of the social order, this command to enjoy actually removes the possibility of enjoyment: "[a]s Žižek points out, when enjoyment becomes compulsory it is no longer much fun” (54). This shift in the structure of the social order and its relation to enjoyment is intimately related to jagodzinski's ethics of violent games. As he writes,

These social changes account for the increase in aggressivity and violence that ... would help to explain the odd inverse between actual violence and video violence as touted by statistics from the Bureau of Justice. The more aggressive the social order, the more need there is to sublimate it through fantasy life. (285) By reinforcing neoliberal capitalist values, media foster an aggressive environment. Advertisements and television programs depict a world in which everyone is enjoying life more than you, fuelling competitive attitudes and feelings of inadequacy which can be expressed in virtual environments. This aggression toward the other becomes sublimated 
and mediated by the screen. Viewing images of violence and death, jagodzinski argues, functions akin to Freud's fort/da game. Television, for example, presents violent images in a mediated form, and through repeated exposure, individuals gain mastery over this traumatic experience:

In a mediated or screened (televised) event, death can only be imagined as a spectacle or representation - as a death of the other. The screen's mediating effects establish a distance between subject and object. It acts as a safety mechanism that allows the viewer to observe and distance a possible threat. When a strict delineated boundary between the source of horror and threat to the self is maintained, the viewer may assert moral superiority and dominance over what is held or represented within the boundary of the frame. . . With this reasoning, the virtual world of televised events should enable a mastery of the sublime to take place, to hold the trauma of anxiety at bay. (287)

For jagodzinski, this process of repetition desensitizes viewers to images of violence: "[r]epeat the horror scene often enough and it becomes tamed by the mind - raising the charge of desensitization, but also enabling the viewer to come to terms with shock" (287).

What he proposes here is very similar to Richard Grusin's theory of premediation, with one crucial difference. Premediation, Grusin states, is a process by which news media make predictions and foreshadow potential tragedies in order to prepare audiences for the worst by remediating the future: "[t]he logic of premediation ... insists that the future itself is also already mediated, and that with the right technologies . . . the future can be remediated before it happens" (Grusin 19). jagodzinski's adaptation of Freud's 
fort/da game and the theory of premediation, however, differ on one point: rather than minimizing anxiety through mastery, Grusin argues that the premediation of the future does not desensitize viewers, but rather induces a constant feeling of low level anxiety. If society is always-already anticipating future tragic events, it will never again experience a shock like 9/11: "the media's preoccupation with premediating the future strives to maintain a low level of anxiety ... in order to protect [society] from experiencing the immediacy of another catastrophe like 9/11" (Grusin 26).

Despite this difference, both jagodzinski and Grusin agree on one fundamental aspect: the effects of viewing these violent images across media is not equal. Grusin suggests that cinematic depictions of tragedy do not premediate the future, as the logic of cinematic and televisual media are radically incompatible due to the structural and psychological differences between media (25). jagodzinski similarly argues that other media, specifically video games, do not mediate violence and death in the same way as television:

Proponents of televisual violence - the broadcast images of death and destruction - can argue that such images enable an ethical response to happen through the sheer intrusion of alterity. ... However, violent video games seem to promote the very opposite. These are not images that can be viewed at a distance.... Alterity is always positioned as a threat to be dealt with. (288)

As jagodzinkski argues, unlike games, where players have an active role in their engagement and interaction with content, broadcast television is a passive one-way transmission to viewers: there is no avatarial relation, no identification with the camera. 
Though this view is a reductive account of one's experience of televisual violence, there is a difference in the way that audiences and players experience acts of violence.

The interactivity of video games presents a unique challenge for theorizing the impact of violence on the player. If video games do not premediate violence, they must, as jagodzinski argues, provide a space for players to act out aggressive fantasies. Yet, jagodzinski further argues that games do not only stage player fantasies, but do so in an environment that is closed to the Real: "in the interactive video game, the intrusion of the Real can be endlessly avoided — again — foreclosing a possible ethical response by doing away with the gap" $(288) .{ }^{11}$ jagodzinski argues that in the absence of the Real, the player's relation to the avatar is no longer mediated, but is instead characterized by immediacy. He states that "the relay time between a player and its avatar appears instantaneous, as if time did not exist” (288). Citing Bolter and Grusin's Remediation: Understanding New Media (2001), he suggests that gaming embodies the dual logic of remediation (hypermediacy and immediacy), such that players experience a kind of "direct unmediated sense experience" (288) in game worlds. The constitutive strategies of remediation consist of both hypermediacy and immediacy — the revealing and concealing of the media form. In hypermediacy, one becomes intensely aware of the medium in which they are engaged; whereas in immediacy, the medium is forgotten and disappears: one believes "that he is in the presence of the objects of representation" (Bolter and Grusin 273). In contradistinction, premediation "differs from the double logic

\footnotetext{
${ }^{11}$ jagodzinski does not appear to be using the term "foreclosing" with reference to the Lacanian term foreclosure, which denotes an absence of the symbolic father which creates a gap in the symbolic: in psychosis, "[w]hen the Name-of-the-Father is foreclosed for a particular subject, it leaves a hole in the symbolic order which can never be filled" (Evans 65).
} 
of remediation in that it represents not a desire for immediacy but rather a fear of immediacy" (Grusin 26).

Consequently, if jagodzinski is correct to assert that the player-avatar relationship is characterized by immediacy, then players exist "on the border between the Real and the Imaginary" (292), putting them at risk of a psychotic break. When the boundary between the Real and Imaginary dissolves, the symbolic becomes foreclosed (in other words, reality loses its structure and meaning) and the subject slips into psychosis. As an example, he states that the cause of the Klebold and Harris Columbine High School shooting was the result of a breakdown between the Imaginary and the Real (296). jagodzinski cites research that finds no causal link between violent video games and individual aggression and violence, and agrees that "[v]iolent games are not the cause of this tragedy; in fact, the fantasy frame actually prevents such tragedies from occurring" (295). Yet, in spite of these assertions, he argues that in the same way that avatars function as a specular image that reflects the player back to themselves, it is also possible for players to reflect and mirror the avatar.

He attributes this unusual reversal of the player and avatar to Žižek's theory of interpassivity. jagodzinski claims that "Žižek, as is often his custom, never defines the term [interpassivity] with precision" (292), and thus he attempts to summarize interpassivity as a phenomenon in which an object actively replaces a passive subject and acts and enjoys in their place (292). "In a strange way," he writes, "the avatar 'enjoys' the game as much as the player does” (292). Unfortunately, jagodzinski fails to fully apprehend the applications and implications of this term, which Žižek discusses in much 
greater detail in his article "Will You Laugh for Me, Please?" (2003). ${ }^{12}$ This oversight leads to his unusual and contentious assertion that, in the case of the Columbine massacre, the two shooters "were heavy players of Doom, an 'ego-shooter' game where the issue of interpassivity is on full display. The figures do all the shooting for the player and the player vicariously lives out the drama on the screen" (294-5). The player-avatar relationship in “"[e]go games' (sometimes called first-person shooter games)”"13 (292) enacts interpassivity in such a way that has the potential to collapse the distinction between reality and fiction, thus triggering a psychotic break in which the avatar assumed the position of the subjects responsible for the shooting:

The psychotic break of the fantasy frame by such young people like Klebold and Harris happens when VR [virtual reality] collapses into RL [real life]. They begin to exist in the gap of the Real. The avatar "takes over" and begins to "control" the player, at times actually addressing the game player to "get even" with the symbolic order that seems oppressively to dominate his or her everyday life. The avatar performatively demands that the evil deed be acted out, an analogue to psychotics who are prone to hear voices in the head. The drives win out over lawful desire. In other words, psychosis lifts the repressions of the Superego, the Law itself, collapsing the fantasy frame that is able to maintain the heterogeneity between VR and RL. (295)

This statement is problematic in its articulation of the three orders in relation to clinical psychosis; if psychosis is triggered via the avatarial relation, why are its effects not more

12 Žižek also discusses interpassivity in "Is it Possible to Traverse the Fantasy in Cyberspace" which appears in The Žižek Reader (1999).

${ }^{13}$ It is unclear why he chooses this term over the more popular first-person shooter genre. 
prevalent; yet, foremost, the root of this position emerges out of a fundamental misunderstanding of interpassivity.

As jagodzinski notes, interpassivity is intimately connected to the shift in the social order from prohibition to commanded enjoyment; however, it is not the avatar that enjoys as much as the player, but rather that the avatar enjoys in lieu of the player. The term originates in the work of Robert Pfaller (2003), who most clearly defines the term in opposition to interactivity; as he writes,

Obviously, the concept of interpassivity is opposed to that of interactivity.

Interactivity in the arts means that observers must not only indulge in observation, but also have to contribute creative "activity" for the completion of the artwork. ... What could be the inverse structure of that? The artwork, then, would already be more than finished. . . . Observers would be relieved from creating as well as from observing. The artwork would be an artwork that observes itself.

In games, interpassivity does not render the player passive, but instead ensures that the player may remain (incessantly) active while still fulfilling the command to enjoy. As an example, Žižek describes the VCR which continually records television shows that he will never have time to watch. In doing so, he can "accede to the Other the passive aspect (of enjoying), while [he] can remain actively engaged — that is, [he] can work longer hours with less need for "nonproductive" activity" ("Will You Laugh”). A more contemporary example would be gamers who purchase countless games from the digital distributer Steam during one of the platform's annual sales, while knowing full well that they will have no opportunity to actually play the games, but nevertheless feel comforted 
that they have fulfilled their duty to enjoy by relegating the passive act of enjoying to the machine. As Žižek critically points out, "[i]n contrast to the notion that new media turn us into passive consumers who just stare numbly at the screen, the real threat of new media is that they deprive us of our passivity, of our authentic passive experience, and thus prepare us for mindless frenetic activity—for endless work" ("Will you Laugh").

Both Pfaller and Zizek's elaborations radically upset jagodzinski's claims about the role of interpassivity in the avatarial relation. It is not the avatar that takes on an active role in place of the player, but rather the avatar is assigned the passive role of enjoying. This interpassive relationship between player and avatar is most explicit in analyses of the conflation of work and play discussed earlier. Work has become a central mechanic of many games. It is common for players to complain about games that require them to excessively "grind" (in other words, perform repetitive and mundane tasks) to gain skill points in order to level up their characters and progress through the game. Yet, work is also a prevalent mechanic in games other than massively multiplayer online roleplaying games (MMORPGs). In Papers, Please (2013), for instance, players work as an immigration officer at a dangerous border checkpoint. Players are required to compare passports and other documents with the country's requirements for entry, and grant entrance to those with complete applications. The player must work tirelessly to approve as many applications (with few mistakes) by the end of the day in order to ensure that their avatar is paid a fair wage to support his family. Although this is a rather literal example, it nevertheless demonstrates the interpassive relationship between player and avatar, wherein the player is incessantly active, and the avatar passively enjoys the benefits of their efforts. 
These assertions directly challenge jagodzinski's claim that the avatarial relation is characterized by immediacy and capable of inducing a psychotic break which inverts the player-avatar positions. As Grusin argues, although "futuristic media technologies might promise to bypass mediation, they don't" (18); therefore, the player's relationship to the avatar is not unmediated, as jagodzinski claims. Furthermore, Freud argues in "Creative Writers and Day-Dreaming" that we do not mistake play for reality: "[t]he opposite of play is not what is serious but what is real. In spite of all the emotion with which he cathects his world of play, the child distinguishes it quite well from reality; and he likes to link his imagined objects and situations to the tangible and visible things of the real world" (1921). This link between play and reality is important, and it echoes Rehak's assertion that "avatars enable players to think through questions of agency and existence, exploring in fantasy form aspects of their own materiality" (123). Play re-stages reality, thereby opening up a space to explore not only fantasy and desire, but also social criticism. Though psychoanalytic theory cannot make definitive claims about how the avatarial relation functions across all games, it can provide an adaptable framework for examining particular instances. As Nusselder writes, "Lacanian theory cannot give simple and unambiguous answers to the question of the psychological impact of information technologies" (102). The following chapter will adopt this framework to examine the relationship between player desire and the neoliberal industry that feeds it, to theorize how games, rather than existing solely as an object of analysis, might contribute its own form of critical theory. Theorists such as Wark and Flanagan have previously theorized the player as a site for critical engagement with games, but this approach, while also necessary, has its limitations. Foremost, the design of one's game shapes how people 
play: Braid, for instance, is not a game to be trifled with, but a more open-ended game like Katamari Damacy invites such behaviour; through exploration and discovery, it encourages the player to disrupt its narrative and goals. In the trifler, Wark sees the potential for critical analysis, but the trifler is disadvantaged by being stuck in the circle of desire. Drawing on Jodi Dean's theory of communicative capitalism, the following chapter will address the obstacles and affordances of games as critical media theory. 


\section{Section IV: Critical Games}

\section{Chapter Seven: Industry, Agency, and Critical Media Studies}

\subsection{Art and Industry}

While a number of theorists have addressed the potential for games as a critical medium, such approaches have been divided, sometimes internally, on whether the responsibility for critical engagement rests with the player or the developer. Both Gonzalo Frasca (2001) and Jane McGonigal (2011) have theorized that as simulations, games can represent real situations to "[foster] critical thinking and discussion" (Frasca, Videogames of the Oppressed ix). McGonigal has explored this potential in her own work by creating games designed to model real world problems, such as in World Without Oil (2007). In this alternate reality game (ARG), McGonigal challenges players to imagine and respond to the fallout from a major oil crisis; as the game's tag line forebodes: "Play it - before you live it." Although appealing, this perspective is idealistic. If we accept that games can have such positive persuasive effects on their players, then we also have to concede that they have negative effects as well. And, as the debate about violent games has revealed, the relationship between games and our behaviour is not so straightforward: there are a number of competing factors that determine this relationship, such as personality, genetics, experiences, beliefs, and culture (Coulson and Ferguson 66).

Such optimistic views of games also tend to overlook the broader industry constraints that determine the kinds of games that can exist. Game making is an expensive and risky industry. With estimates of development costs of popular game titles ranging from 20 to in excess of 60 million dollars and rising (Superannuation), it is no wonder that AAA companies like Take-Two Interactive, Electronic Arts, Activision, and 
Ubisoft Entertainment are hesitant to put unproven projects into production. As Tracey Fullerton pointed out in 2008, the top twenty games generate $80 \%$ of sales (423). In 2010 , Mateos-Garcia et al. estimated that only $20 \%$ of games that are released will cover their costs, which is exacerbated by the fact that, as they state, "[t]he escalation in budgets has not been accompanied by corresponding increases in the size of the market" (7). The issue of development cost, in other words, is compounded by a significant increase in the number of games produced each year. The creator of Steam Spy, Sergey Galyonkin, found that "38\% of all Steam games were released in 2016" (@Steam_Spy 30 Nov. 2016), totalling 5069 games (@Steam_Spy 14 Sept. 2017); as a comparison, 736 films were released in the US in 2016 (Follows). According to Galyonkin's estimates, the number of games released each year will continue to rise, ${ }^{14}$ despite measures taken by Steam to regulate "fake games," subpar money-grab releases that are also known as shovelware (Frank). These "games" are not developed for players to purchase, but are designed for bots that are capable of taking advantage of Steam's card-trading platform to generate income through microtransactions.

Excluding shovelware, the issue with the increasing number of game releases is that it exacerbates the challenges that independent developers face in trying to survive. The result is a market that is strictly driven by consumer demands. Developers must meet demands for features like increased agency, meaningful choices, and multiplayer, while standing out among the thousands of games released that year. Should developers fail to meet players' expectations, their game could face a barrage of negative customer reviews,

\footnotetext{
${ }^{14}$ According to Galyonkin, 7,672 games were released on Steam in 2017 (@Steam-Spy), or $41 \%$ of all games on Steam.
} 
which can be fatal in a market that has become suspicious of game journalism since the events of GamerGate in 2014. A particularly infamous example of a game's subjection to the wrath of its fans is Hello Games' No Man's Sky (2016). While critic reviews were positive but somewhat underwhelmed, players rated the game significantly lower. ${ }^{15}$ As Carolyn Jong notes, "Gamers often justify their actions by claiming that their status as the primary consumers of games entitles them to 'criticize' developers or journalists who fail to live up to their desires or expectations. Frequently, however, these criticisms slip into personal attacks, and are disproportionately directed at women and minorities" (6). No Man's Sky's developers have been fighting an uphill battle to overcome negative reviews from fans and even death threats (Good). Attempting to assuage their critics, developers have released a number of free updates that contain content and features demanded by their most vocal fans. Currently, $63 \%$ of the game's reviews are negative, with more recent reviews described as "mostly positive."

In this economic environment, it is no surprise that the largest game companies would produce proven content to maintain sales. Rebooting popular shooters and updating rosters for sports franchises each year is a reliable way to maximize profit with minimal cost and risk. As a medium, games are in their adolescence, and it is still difficult to determine not only what projects will be successful, but also how to support ventures into new territory, which are financially riskier than maintaining the industry standards and replicating successful franchises. As Mikolaj Dymek notes, the top ten console games each year tend to be sequels and variations of existing franchises:

\footnotetext{
${ }^{15}$ Metacritic.com shows a critic rating of 61/100 and a user rating of 2.6/10 for No Man's Sky.
} 
the video game industry thrives on this limited list of highly formalized and rigid game genres and has done so practically since the 'golden age of video games' in the 1980s. The industry continues churning out endless number of sequels, copycat games 'inspired' by successful competitors, quick-and-dirty movie-based games of subpar quality, 'content incrementalism' (usually sport titles) and other path-dependent genre strategies. (41)

Although repetitive, this formulaic approach to game production offers more predictable revenue streams.

Given the precarious state of the industry, critical games that challenge player expectations and conventions are not simply less likely to succeed, but less likely to go into production. As a result, the burden for critical engagement falls to the player. This is not an unfamiliar state of affairs. In Critical Play (2009), Mary Flanagan notes that children have always been critical players who subverted the intentions of their games and toys. Her concept of "critical play," while too broad for the needs of this chapter, captures the critical interplay between design and interaction. As she writes, "[c]ritical play means to create or occupy play environments and activities that represent one or more questions about aspects of human life" (6). Examining the history of dollhouses, Flanagan explains that

Play housekeeping ... acted as a continuous symbolic reinforcement of Western household tradition, and the practical and ritualistic function of housekeeping became a desired focus of play. Inexpensive to make and prudently imaginative in their recycling of materials, the scrapbook houses could be made by anyone 
who could afford time, paste, and scissors, making the trend a democratic form not only of play but also of normative reinforcement. (30)

Children frequently engage in critical play with toys in ways that Flanagan describes as unplaying, reskinning, and rewriting (32); as an example, she notes that, "[i]n doll play, unplaying manifests in children abusing their dolls, 'killing' them, or some other revision of the 'care giving' framework of expected play. This critical kind of play reverses traditional expectations regarding care-giving behaviors and allows players to rethink the conventions involved in these social roles" (33).

This idea that players do not always play with toys and games as intended easily extends to digital games. In Gamer Theory (2007), McKenzie Wark draws from Bernard Suits' The Grasshopper to name those who do not recognize the goals of the game "triflers." These players are distinct from cheaters in that the cheater absolutely recognizes the goals of the game, but does not play it according to its rules. The trifler, however, has little interest in winning the game, and instead explores the game through its rules and limits. As such, triflers are best suited to critical play because they work within the game's system to challenge its meaning: "[i]n gamespace there may be no idler, but there may be what Bernard Suits calls the trifler, who plays the game according to its rules but not aiming for its prizes. The possibility for critical thought is now internal to the game, to its rules, to its spaces, its conventions, but not to its goals" (Wark 234, endnote 034). Wark's statement echoes Flanagan's conditions for critical play in that like children who undermine the lessons of domesticity embedded in playing house, the trifler rejects the intentions and goals set by the developer. 
Not all games, however, lend themselves to the kind of trifling and critical forms of play that Wark and Flanagan imagine. Wark notes that a game focused on winning, like State of Emergency (2002), is an unlikely site for trifling (167). In such a game, players become caught up in climbing leaderboards, and collecting achievements, points, and items. Similarly, online multiplayer games function like a kind of self-regulating machine that enforces particular kinds of normative play as conventions and strategies are developed through player interaction. Players who transgress these conventions are either reprimanded by other players, or if their actions are deemed to be cheating, they may be banned from the game. It was recently announced that the online multiplayer battle royale game PlayerUnknown's Battlegrounds (PUBG) (2017) banned 1.5 million of its players for cheating (Hester). These obstacles raise important questions about the conditions necessary for games to function as critical media. If the mechanics of a digital game can discourage or suppress critical play, then the power to facilitate these conditions must be held by developers — the burden returns full circle.

Extending her work on play to digital games, Flanagan asks, "what if certain games have become something more? What if some games, and the more general concept of 'play,' not only provide outlets for entertainment but also function as means for creative expression, as instruments for conceptual thinking, or as tools to help examine or work through social issues?" (1). Some games certainly do embody the kind of critical play that she imagines, but such projects are economically risky, even if they are desired. Given trends in games and the entertainment industry more broadly, it is unlikely that the kinds of critical works that theorists desire will emerge from the large risk-averse studios and publishers that dominate the industry. A call for critical works, therefore, 
inadvertently puts the burden of their creation on independent (indie) developers. As Allison Harvey notes, "indie game-making in particular is mapped in opposition to the ideology of the mainstream industry, offering enlightenment in the face of market-based efficiencies" (Harvey 96).

In recent years, the proliferation of user-friendly game development software has made it possible for users with little or no programming knowledge to create and distribute their own games and interactive fiction. Development tools such as Game Maker and Twine have enabled users to create interactive and multi-linear narratives that defy the constraints of oral and print traditions. Twine, in particular, has stood out as a tool that has been central to diversifying the game community, due to its accessibility. These new tools are changing not only how stories are told, but also the kinds of stories that are told. The communities that have rallied behind these resources reveal that there is a strong desire for critical games. Discussing the Twine revolution, Harvey both celebrates and problematizes this movement; as she explains,

What these sites and practices indicate is a way for digital game designers who have been excluded from the mainstream system to create, expand, and promote their own economy of production. However, the precarity of these practices, particularly the modes of funding, is striking. They tend to be contingent on goodwill, just enough, [sic] temporary measures, a reality that begs the question of sustainability and the livelihoods of these game designers. (104)

Within the indie and amateur game movement, it is generally expected that games will be made available at very little or no cost. Speaking about her own situation, Anna Anthropy writes that "[t]he problem with Patreon — and Kickstarter, and crowdfunding in general— 
is that mostly what it accomplishes is to recirculate money within marginalized communities. It's mostly other queer people who make slightly more than me who are paying me every month. Crowdfunding rarely brings in the money of those who actually have it" (45). The narrative of the "starving artist" is widespread, and for games, this labour of love has led to the exploitation of developers and programmers in both the indie and AAA industries. Despite their promises, such forms of public funding do little to repair the status of games as commodities.

Addressing the situation in AAA companies, Nick Dyer-Witheford and Greig de Peuter's Games of Empire (2009) offers a snapshot into the life of an employee at Electronic Arts, where crunch time is so normalized that 100-hour work weeks are not out of the ordinary (59), where overtime is often unpaid, and when they are home, developers provide continued unpaid cognitive labour; as one unnamed game studio executive stated: “[u]nlike machinery that stops working at 5:00, ours might be home, [but] they're thinking of new ideas, and their whole life experience is creating the potential for new ideas" (qtd. in Dyer-Witheford and de Peuter 37). They argue that the industry is fuelled by cognitive capitalism: "a system of production in which knowledge plays the integral role" (36) and "where workers' minds become the 'machine' of production, generating profit for owners who have purchased, with a wage, its thinking power" (37).

In recent years, however, there has been a small exodus from large studios as developers leave their relatively stable AAA jobs to pursue their own indie game projects (Dyer-Witheford and de Peuter 42; Grubb). The narrative that sustains this shift promises that developers will succeed if they are willing to first sacrifice everything in order to 
fund their vision. The release of Canadian-made Cuphead (2017) provides an ideal illustration of the fulfillment of this dream:

Perhaps echoing Cuphead and Mugman's deal with the devil, the Moldenhauer brothers went all-in: They quit their day jobs and remortgaged their houses to work on the game full-time.

"From the outside looking in, it definitely seems like a huge risk," said Moldenhauer. "Our opinion of the reception was we should be able to just break even and still make this perfect game that we want. We might both be slightly crazy, but that's how we felt.” (Ore)

Such stories of success fuel the fantasy that if one has the initiative and resourcefulness, one will succeed as an indie developer. Addressing the lived experience of indies, Galyonkin challenges this narrative, explaining that "[e]verywhere else small creators face the same problem: making the ends meet with a relatively small audience. It's never easy for a small guy and it will never be, but in most other industries indie products are still sold at the same price as big AAA titles from established brands and companies." As he discovered by analyzing data from Steam, "[a]n average indie title these days is priced $\$ 8.72$ on Steam. During the last Summer Sale [2016] the price went down to the mindboggling $\$ 4.63$ and yet the average sales are at their lowest at 21,000 copies." In comparison, the cost of a new AAA title game is $\$ 79.99$. Independent game development is seen as a haven for both senior and amateur game creators who want the freedom to explore their creativity, which is often squandered when working for large companies. Free from industry constraints, indie developers can explore new subjects and tackle traditionally taboo topics. Games such as Toby Fox's critique of violent games in 
Undertale (2015), or Zoë Quinn's Depression Quest (2014), a game that disempowers the player to share her experience with depression, demonstrate the power of games to challenge mainstream conventions and offer a critical take on games. However, it is imperative to recognize that these games are the exception and that it is problematic for the burden for critical works to be placed on this already struggling community of developers when there is a clear demand for such games. The financial reality of being an indie is unforgiving for those who want to pursue a career in games. As Bart Simon notes, although there is little to gain in delimiting the category of what constitutes an "indie game," one can be sure that "[t]o speak of an indie game, unlike other video games, is to speak, often very passionately, about the context of the production of that game. To speak of indie games is not to speak only of the games themselves or of the experiences of gameplay but rather of the cultures of game development from whence they came" (2). Examining the postmortem of Question's The Magic Circle provides a case study for addressing these very conditions.

\subsection{On Making Games: The Magic Circle}

In Gaming: Essays on Algorithmic Culture (2006), Galloway deemphasizes the player's role in the discussion of games as a critical medium, imagining instead a kind of transgressive game through what he calls countergaming. Following the tradition of counter-cinema, Galloway describes countergaming as a site of resistance to conventional aesthetics, narrative, and mechanics. Through such innovative and experimental games, the movement would realize "its true potential as a political and cultural avant-garde" (126) and would redefine play through radical action, a term that he uses to describe 
practices of countergaming that rethink a game's mechanics, gameplay, and algorithms $(125)$.

The independent production of games promises to be a space for exploring the aesthetic and political potential of the medium free from the constraints of market demands and player desires. The supposed freedom offered by independent production, however, is questionable, as those who left AAA jobs to pursue independent projects have learned. It is clear that this movement has encouraged a number of developers to push the medium into new territory, as in recent years, many games have been released that explore themes like political revolution and social critique. The financial state of the industry, however, makes the future of these games unsustainable.

At the 2016 Game Developers Conference (GDC), the team behind The Magic Circle (who had previously worked on games such as BioShock and Dishonored) discussed their game's sales on a panel about whether the indiepocalypse, if it exists, was to blame for the game's poor sales; in other words, was the market simply oversaturated with indie games, or did they "shiv" themselves, as he says, by making a game with no clear genre that was too weird and too meta. The title of this game is a direct reference to Johan Huizinga's concept of the same name, which is used to describe a social agreement that sets game space apart from the real world (Huizinga 10). The magic circle is what turns kicking a ball into scoring a goal. While the usefulness of this concept has been subject to critique and debate (Consalvo 2009), the game borrows this term to highlight its own exploration of these boundaries as they are set by developers and reset by players.

The game situates the player as a playtester for an unfinished game sequel that has been in development for twenty years. Its environment is nearly devoid of colour, and 
developer notes left scattered throughout alert the player to issues with mechanics, inconsistencies in the world design, and problematic puzzles. The game's development is fraught with conflict: its progress is stifled by its auteur creator, Ishmael Gilder, who envisions his masterpiece as a linear, narrative-driven adventure, while the game's team pushes for increased combat and player agency. Ish is hostile to these requests, as he sees the player's ability to act in the world as an obstacle to storytelling. In the player's first encounter with Ish, he removes the player's weapons on the basis that they will take away from the narrative that he has so carefully crafted; as Ish explains, players "demand a world full of deep, compelling characters, yet at any time, they reserve the right to turn said people into piles of gently glowing ash! What possible story could survive these conditions?" (The Magic Circle). The game's narrative is a hyperbolic manifestation of the ludology vs. narratology debate, where the player is caught between two project leads, one who emphasizes a story-rich experience, and the other thrilling gameplay. Witnessing the passion behind each vision for the game, the player is put in the uncomfortable position of critically reflecting on the challenges of game design, including how their own desires for features that allow them to shape its world and narrative come at the cost of a compelling experience crafted by developers.

The development of the putative game is further hindered by the failure of its most recent Kickbackr campaign, which fell far short of meeting its goal as a result of fans being fed up with the game's many delays. Guided by the "old pro," an artificial intelligence that was abandoned in an earlier iteration of the game, the player works 
behind the scenes to ensure that the team has a demo ready in time for E4. ${ }^{16}$ While the fictional development team is able to debut their work at the event, outraged fans interrupt the show and demand that the game's development be turned over to them. The protesters imagine that by using the fan fiction that they've written over the past two decades, they will be able to collaboratively develop a better quality sequel. Surprisingly, Ish relinquishes his control over the game to his fans, and its development is opened to the public. Their collaboration does not go so well, however, and their efforts quickly devolve into chaos.

Through this unusual narration, Question reveals that the magic circle is broken before a game is released into the world. Developers are always already thinking about and designing for players who will not necessarily play the game as intended. As the game's narrator states,

The gods [developers] claim every world begins with a circle. Inside this line, whatever they say - goes. By just plain existing here, you agree that white is good and black is bad, auto-friggin-matic. So I asked myself, what happens when some wise-ass draws his own circle inside theirs? Things get real meta. So buckle up. (The Magic Circle).

The Magic Circle clearly challenges the idea that games are in any way separate from the operation of everyday life; play and work, or games and industry, are not as distinct as we once believed them to be. The aim of this chapter, however, is not to level a critique of Huizinga's magic circle, but rather to examine more closely this point at which "things

\footnotetext{
${ }^{16}$ A play on E3, the annual Electronic Entertainment Expo where companies and developers showcase upcoming products.
} 
get real meta." Foremost, The Magic Circle is a game about making games. It highlights a number of the challenges that developers face: fans, crowd funding, promotional events, deadlines, bills, and striking a balance between creativity and player demands. It is a game about how developers either are trapped as cogs in cognitive capitalism, or must struggle to produce works that might satisfy their creativity, but will not financially support them.

In his analysis of the "Cycles and Circuits of Struggle in High-Technology Capitalism," Dyer-Witheford applies an autonomist Marxist theory to argue that the development of capitalism moves in cycles as it is shaped by workers' struggles (196). A "cycle of struggle" denotes a moment in history where workers unite against employers to improve their working conditions to which capital responds by adapting and neutralizing that resistance. Capital depends on economic inequality, so instead of eliminating workers, they are recreated in a new form. A simplified version of this cycle begins with a skilled worker who realizes the value of his contributions and demands better wages. Instead of conceding to these requests, assembly lines are created that divide the duties of the skilled worker among many unskilled workers; this economic system is known as Fordism. When these unskilled workers inevitably revolt against their working conditions, capital relocates these jobs to countries with fewer regulations through innovations in information and communication technologies; consequently, globalization emerges as an effect of capitalism. As Dyer-Witheford writes, "capitalism resorts to incessant technological renovation as a 'weapon' against the working class" (196). He refers to the current cycle of struggle as that of the "socialized worker," one who's labour and leisure time are closely integrated. The socialized worker is central to 
cognitive capitalism. For the socialized worker, as discussed in Chapter Six, daily life and work are no longer clearly separated, as productivity and leisure time have become conflated. This makes for longer and more stressful work days that are never ending. In the struggles of the socialized worker, it is easy to draw a parallel to the current working conditions of game developers discussed in Dyer-Witheford and de Peuter's Games of Empire. These workplaces are often designed to be fun (much like The Googleplex or Apple Park), but, as Dyer-Witheford and de Peuter point out, "[t]he irony ... is that the very attractions that make employment in video games 'not so much like work' can also turn it into a digitized iron cage and convert a dream job to a nightmare" (56). Employees should feel lucky for having the opportunity for working on successful AAA titles, and if not, they are easily replaced by other eager, young programmers. In this not-so magic circle of struggle, developers become unable to challenge their working conditions and are thus trapped in what were supposed to be dream jobs.

The developers of The Magic Circle, having worked on franchises like BioShock, Dishonored, and Deus Ex, found themselves in this very situation, and so they quit their relatively stable jobs and risked their livelihood to create their own independent studio to free themselves from the constraints and obligations they faced in their AAA jobs. Unfortunately, the studio's first and only release so far did not meet sales expectations. Four months after the game's release in 2015, it had only sold about 16,000 copies, but it was found on the wishlists of 44,000 Steam users. At GDC 2016, one of the game's developers, Jordan Thomas, spoke on a panel about whether the game's failure was the result of a boom and bust cycle of the industry, or whether the game's unique and selfreferential content was to blame for its poor sales. 
Although reviews for the game are largely positive,${ }^{17}$ it is criticized for its short playtime and cost; as Steam users know, if they wait, the game will be available at a lower price during one of the platform's annual sales. Accordingly, Thomas warns his audience that if developers want to make a risky project, it should give players a reason to buy the game immediately, or they risk being forgotten on wishlists. Concluding his talk, he says that the time they spent on The Magic Circle "claimed [their] innocence," and that their next game, The Blackout Club (forthcoming 2019), will play it "safer" and include features that players demand in order to improve sales, such as cooperative play and procedurally generated adventures. The approach to game development outlined in Thomas' talk stands in stark contrast to The Magic Circle's critical take on fans, participatory culture, and the industry. Participatory culture is an increasingly important part of the game industry; this trend is evident not only in AAA games, but also in the production of smaller independent games that rely on crowdsourcing and funding through early access development and sites like Kickstarter to gain support for their projects. These resources allow players to provide early feedback that developers can incorporate into the game before its release. The Magic Circle did not employ the help of Kickstarter because they felt it was at odds with the game, which criticizes the use of such platforms on the basis that financial investment by fans takes away from the developer's creative freedom, making them beholden to an audience. As the game's conclusion reveals, it is not possible to mediate the discordant demands of fans.

As a game about games that grapple with the pressure of participatory culture, The Magic Circle's critique is echoed in Thomas' talk when he suggests that developers

${ }^{17}$ As of March, 2018, 91\% of reviews are positive. 
feel burdened by players' demands for features like divergent endings, increased agency, and multiplayer experiences; and yet, the description of the studio's next game suggests that the team has accepted defeat. This view of participatory culture, however, is not shared by all. Game theorists such as Henry Jenkins and Jane McGonigal argue for the benefits of fan participation. Participatory culture is an increasingly important part of the game industry; this fact is evident not only in AAA games, but also in the production of smaller independent games through Kickstarters and Early Access games that develop in response to player feedback. Jenkins and McGonigal believe that there is an inherent good in investing in these kinds of participatory and collaborative projects. As Jenkins' writes, drawing on Grant McCracken, "in the future, media producers must accommodate consumer demands to participate or they will run the risk of losing the most active and passionate consumers to some other media interest that is more tolerant" (133).

Independent developers, however, are less certain about these trends, as it is precisely this drive for consumer inclusion that has pressured them to return to the values of the AAA game companies that they had left. Rather than using the indie market as a venue for exploring their creative potential, these developers have found themselves in a position that is all too familiar.

By juxtaposing the desire to participate with the desire for compelling narratives, The Magic Circle produces a situation analogous to what Miguel Sicart calls a "wicked problem:" a situation with no optimal outcome that invites critical reflection. As Sicart argues, these moments facilitate a kind of player engagement that goes beyond winning the game: "[p]layers care if they are challenged to care and if play becomes more about questioning a system than interacting with that system" (108). The Magic Circle invites 
players to look critically at current trends in the game industry through Ish's passion for creating a story, through the desire of the team to create an enjoyable play experience, and through the fans' dedication to the series. Question reappropriates and recontextualizes a fictional AAA title as the setting for its indie game to level a critique of the industry, but it ultimately presents a balanced view that highlights the value of both creator driven single-player experiences and participatory culture; the issue, the game suggests, is when the two are forced together in the interest of sales and profit margins. When the fans' self-sabotage their attempt to collaboratively re-design Ish's game, its development is handed over to the player by the narrator. The player is transported to a level-editor where they must construct a world, and place NPCs, enemies, loot, and music to finish the game and release it to the public. Although the ending of the game is truly imaginative and engaging, it undermines the game's message. Up until that point, the game resists participatory culture by revealing its inherent paradoxes, until its conclusion, which tells the player that they will be heard in the cacophony of voices, and that their contribution is important and will save the sequel. It is a similar fantasy that drives indie developers: they believe that out of the 7,672 games released on Steam, theirs will be seen, bought, and validated.

The Magic Circle, at least momentarily, invites players to take a critical look at the industry by using the medium in an unexpected way, but its conclusion undermines its critique by privileging the role of the player in the end. While games that cater to players through customizable features, multilinear storytelling, and open worlds may make exciting games, they do not provide the best site for exploring the medium as a critical art form, as they perpetuate problematic trends in the industry. We must ask, 
however, what will be lost if developers design for maximizing sales by handing over control of the game world to players; what kinds of experiences will players be compelled to create? I argue that these player-centric trends not only pose an obstacle to games as a critical medium, but also fuel what Jodi Dean has named communicative capitalism.

\subsection{Communicative Capitalism}

In the drive to participate in and across media, Jodi Dean recognizes dangerous political implications that she addresses through her theory of communicative capitalism. In brief, communicative capitalism designates "the merging of democracy and capitalism in mass personalized media, the networks of mobile phones, wifi, social media, and mass distraction through which we circulate our feelings and opinions in ways that make us feel important, engaged, [and] political" (Dean, "Not Us, Me"). This mode of communication is most explicit in the success of sites like Facebook, Twitter, and others that are sustained exclusively by user-generated content. The commodification of communication, she argues, encourages the rapid circulation of images, ideas, and affects in order to distract us "from the antagonisms ... of contemporary society, inviting us to think that each opinion is equally valid, each option is equally likely, and each click is a significant political intervention" (Blog Theory 2). The result is the "suspension of narratives, patterns, identities, [and] norms" (31); if all opinions are equal, then there is no longer an authority over meaning. The circulation of fragmented and digestible opinions and affects not only facilitates the distribution and consumption of communication, but also safeguards communicative capitalism against critique. The 
nature of commodified communication leaves little time for sustained critical thought, as the rapid circulation of information necessitates hasty reactions to events before the conversation shifts. Consequently, as Dean argues, communicative capitalism "captures critique and resistance, formatting them as contributions to the circuits in which it thrives" (2), until critique becomes just another opinion among many.

Working in Lacanian terms, Dean attributes the success of communicative capitalism to the decline of "symbolic efficiency": "aka the collapse of the big Other" (5) .Without the authority of the big Other, meaning itself becomes unstable: “[t]here's always another option, link, opinion, nuance, or contingency that we haven't taken into account, some particular experience of some other who could be potentially damaged or disenfranchised, a better deal, perhaps even a cure" (6). For Dean, this radical uncertainty signals a shift in the subject's position from the circuit of desire to the circuit of drive. Unlike the circuit of desire, which propels the subject forward from one object to another in search of jouissance, drive is an "excessive, repetitive, and ultimately destructive" (Evans 48) path that traps subjects into performing the same actions and patterns again and again. Like a zombie's impersonal pursuit of brains, drive instigates an incessant repetition of a behaviour that delivers enjoyment from its own failure to achieve its aim; as Dean argues, when subjects are captured by drive they get "stuck doing the same thing over and over again because this doing produces enjoyment. Post. Post. Post. Click. Click. Click" (40). Repetition, in combination with the decline of symbolic efficiency, produces individuals that are no longer subjects proper, but have instead become "whatever beings," a term that she borrows from Giorgio Agamben to describe individuals that belong to nothing in particular, who have no identifiable politics, and no 
symbolic identity. The fantasy of participation drives communicative capitalism by justifying our behaviour. People are eager to participate because it conceals all of the ways that we are not able to adequately participate in important political decisions. It ensures our participation despite our suspicions that a single vote is inconsequential.

In a world dominated by opinion, sustained critical thought is relegated to the background as simply another opinion among many. To challenge communicative capitalism, Dean asserts that a critical media theory is needed that resists the fragmentation and rapid circulation promoted by communicative capitalism; such a form, she proposes, is the book, which can enact the strategies used in workers' struggles to challenge and disrupt the reader. As she writes, "critical media theory is possible in book form. The wager is inspired by a time-honored tactic in workers' struggle: the slowdown. As an object whose form installs delays in sampling and syndication and whose content demands postponed gratification, the book mobilizes the gap of mediacy so as to stimulate thought" (3). The time-consuming nature of the book is thoroughly incompatible with the values of communicative capitalism; however, she is not so quick to extend her theory to include electronic forms of writing like e-books or blog posts, as these media are more easily captured by communicative capitalism: the critical affordances of the book's design are exclusive to print in her view. The book demands one's full and dedicated attention, its form is slow to produce, and its content frustrates its readers with lengthy diatribes and expositions, thereby indefinitely deferring the instant gratification desired by "whatever beings." As Dean outlines, "critical media theory is possible when it occupies the trap of its emergence, not when it offers happy solutions (the happiness of which would necessarily belie the severity of the problems it 
diagnoses) and not when it presumes that an analysis of entrapment or capture is the same thing as escape (which would presuppose that the trap was a pseudo trap of words all along)" (32). It is precisely because the book is too slow to respond to events and timeconsuming to read that it is best suited as the site for critical media theory.

But is the book the only medium that can break the drive's hold on its subjects? After an engaging critique of contemporary society and an elaboration of the role of drive in the perpetuation of affect and opinion, readers are left with the book (perhaps even Dean's e-book) as the only tool to resist or defend against communicative capitalism. In Infinite Distraction (2016), Dominic Pettman writes that, "[t]he very decision to write 'a book' about interactive networks presumes the capacity to manufacture an object somewhat removed from that milieu, in order to have the necessary distance and perspective to write about it (even as the book is likely to end up as a pdf file online soon after publication)" (Pettman 14). Is it reasonable to think that the book is in any sense removed from the digital at any point? A book is almost inevitably typed on a computer, early forms are often released as snippets in online journals, ${ }^{18}$ and the final print product is often bundled with a digital copy.

This rejection of digital media is problematic in that it repeats the tired response of culture jamming to capitalism that theorists like Henry Jenkins critique as pessimistic and unproductive (248); as he writes in Convergence Culture (2006),

Too many critical pessimists are still locked into the old politics of culture jamming. Resistance becomes an end in and of itself rather than a tool to ensure

\footnotetext{
${ }^{18}$ For instance, an early version of Dean's work on communicative capitalism appears in Culture and Politics.
} 
cultural diversity and corporate responsibility. The debate keeps getting framed as if the only true alternative were to opt out of media altogether and live in the woods, eating acorns and lizards and reading only books published on recycled paper by small alternative presses. But what would it mean to tap media power for our own purposes? (248-9)

Jenkins believes that individuals should fully embrace and participate in popular culture to unlock its political potential as a participatory democracy. Taking an analogous approach, Nick Dyer-Witheford argues that Marxist resistance to capitalism should incorporate both refusal (sabotage) and reappropriation (197). While the book may facilitate the kind of slow-down that disrupts drive, Dean's assertions concerning the suspension of narrative forces critical media theory to take a limited form that leaves no room to benefit from existing technology. The book may be a promising site for resisting communicative capitalism, but given the "publish or perish" state of academia, it is not especially inspiring. There is no shortage of critical and scholarly texts, and for that reason, one must challenge the idea that the book, as an object, can extract subjects from the grasp of the drive.

Occupying a position between Dean's and Jenkins' opposing views, I argue that it is not the book itself that holds this potential, but rather the kind of interaction that it facilitates with readers. Drawing on a more inclusive definition of narrative creates a space for games as another site of resistance that facilitates the same conditions and tactics that make the book a valuable site for critical media theory. Dean argues that blogging, Twitter feeds, and other ubiquitous digital media do not produce narratives, but instead create a corpus of disconnected messages and posts having more in common with 
a database: "Words matter, not stories and not narratives" (89). Blogging, she writes, “isn't narrativized. It's posted. It's not told as a story but presented in moments as an image, reaction, feeling, or event" (47). This perspective of narrative borrows from Lev Manovich's work in The Language of New Media (2002), wherein he argues that “database and narrative are natural enemies" (225). Manovich's claim, however, hinges on an Aristotelian understanding of narrative that privileges order, chronology, and causality. As Rick Altman elaborates, for narratologists who follow Aristotle, "all narratives have a beginning, middle, and end. . . Narrative definition is ineluctably bound to plot, and the plot in question is always of a particular kind" (5). This conception of narrative, however, has been challenged by theorists such as Rick Altman, MarieLaure Ryan, and Janet Murray, to name a few. Ryan, for instance, aptly critiques Manovich's claim by arguing that when presented with randomized fragments of text (stored in a database), readers will "mentally reorder the information into a coherent narrative" ("The Interactive Onion" 40). As an example, one might consider the sequence activities found in children's workbooks that ask children to arrange a series of images into a linear order. Just as children are able to quickly rearrange the pictures into a coherent narrative, so too is it possible to mentally reorder the events of a more complex story. These new narratives may very well be contained in a database (particularly in the case of hypertext fiction), but one is nevertheless able to experience its contents as narrative. Employing a more flexible definition of narrative reveals not only the capacity of these disorderly and fragmented narratives to disrupt drive, but also their ability to serve as a kind of critical media theory. Unlike the fragmented posts of social media, the 
content of such a narrative is not immediately apparent. One must work to reassemble its meaning, and unlike social media, one is complicit in this activity.

In How Games Move Us (2016), Katherine Isbister argues that games offer players a whole new emotional palette of experience that is not possible in other media (8): player agency enables games to evoke feelings of pride, guilt, frustration, camaraderie, and complicity (41). For Dean, the affective dimension is crucial to capturing the subject in communicative capitalism; for this reason, she argues, "a media theory that is critical has to foreswear the affective enterprise of contributing the feelingimpulses of hope and reassurance and offer thinking instead" (32). While narratives can evoke powerful feelings to draw audiences into their stories, the ability to act in a game world creates in the player a sense of responsibility and feelings of pride or guilt over the events that take place. These affects can be harnessed to engage players in critical thought.

To illustrate what a kind of critical media theory of games might look like, I offer two examples by Davey Wreden, The Stanley Parable (2013) and The Beginner's Guide (2016). These games offer a critique of player agency, the game industry, and the role of art, and they do so in a way that, I argue, conforms to Dean's conditions for critical media theory in the era of communicative capitalism.

\subsection{Agency and the Critical Role of Narrative: The Stanley Parable}

In The Stanley Parable, Wreden offers both a critical and reflexive account of the relationship between agency and narrative by putting the player in conflict with the game's omnipresent and often overbearing narrator. Unlike the algorithmic and 
character-building approaches to multilinear storytelling in The Novelist and The Walking

Dead, The Stanley Parable builds an environment that accounts for all possible actions and directions that can be taken by the player. Wreden's narrative structure produces approximately nineteen different outcomes. Players take on the role of Stanley, an office worker whose job entails entering instructions into a computer, until one day the instructions stop and Stanley discovers that everyone in his office building has disappeared. Guided by the narrator, the player sets out to explore the building and uncover the mystery. The narrator directs the player to the office meeting room, and, as Stanley comes to a set of two open doors, the narrator states that Stanley enters the door on his left. Here, players may choose to act in discordance with the narration and enter the door on the right. ${ }^{19}$ Should players continue on this defiant path, the narrator becomes increasingly irritated and pleads that he only wanted to show the player "something beautiful." The player, however, may choose to subvert the narrative entirely by ending the game by falling from a platform, to which the narrator responds, "in his eagerness to prove that he is in control of the story and no one gets to tell him what to do, Stanley leapt from the platform and plunged to his death. Good job, Stanley. Everyone thinks you are very powerful" (The Stanley Parable). The narrator's rejoinder accurately captures the tone of the game, which frequently mocks and pokes fun at the player's desire for agency. Unlike other games that offer the player agency, Wreden's game actively taunts the player for making meaningful choices.

${ }^{19}$ A study by Scanlon et al. investigated whether players responses to this situation were affected by the gender of the narrator, and found that women were more likely than men to disobey the narrator when choosing a door (3). 
Unlike BioShock Infinite, which maintains the illusion of agency through the coexistence of multiple and conflicting (inaccessible) worlds, Wreden's game puts the player in opposition to the narrator to call the player's agency into question. In so doing, it criticizes recent trends that push developers to create games based on consumer demands. If the player resists the narrator's instructions at every opportunity, the narrator becomes frustrated that the player has refused to play The Stanley Parable as he intended, and demands to know what would have improved the player's experience: vehicles, skill trees, leaderboards, or more choices. After exploring a few of these options, the narrator offers to let the player try his new art game in which a baby crawls toward danger and the player must push a button to send the baby back to the start: "It's a very meaningful game," the narrator explains, "all about the desperation and tedium of endlessly confronting the demands of family life. I think the art world will really take notice. But, of course, the message of the game only becomes clear once you've been playing it for about four hours. So why don't you give it four hours of play to make sure it's effective. Be sure to keep notes on your experience." If the player successfully pushes the button repeatedly for four hours, the player will be shown a message that expresses love for the player and a few other odd validations. However, in the very likely instance that the player fails to push the button for the full duration, the narrator becomes offended and states that he will no longer create games for the player, and will only make games to "fulfill a greater artistic purpose." The narrator then forces the player to play short demos of popular games like Minecraft and Portal, which he quickly finds dissatisfying. Before abandoning the player, the narrator states that he does not need the player's validation, 
but rather it is the player that needs the narrator to "wrap everything up at the end, to make sense out of the chaos, the fear, and the confusion."

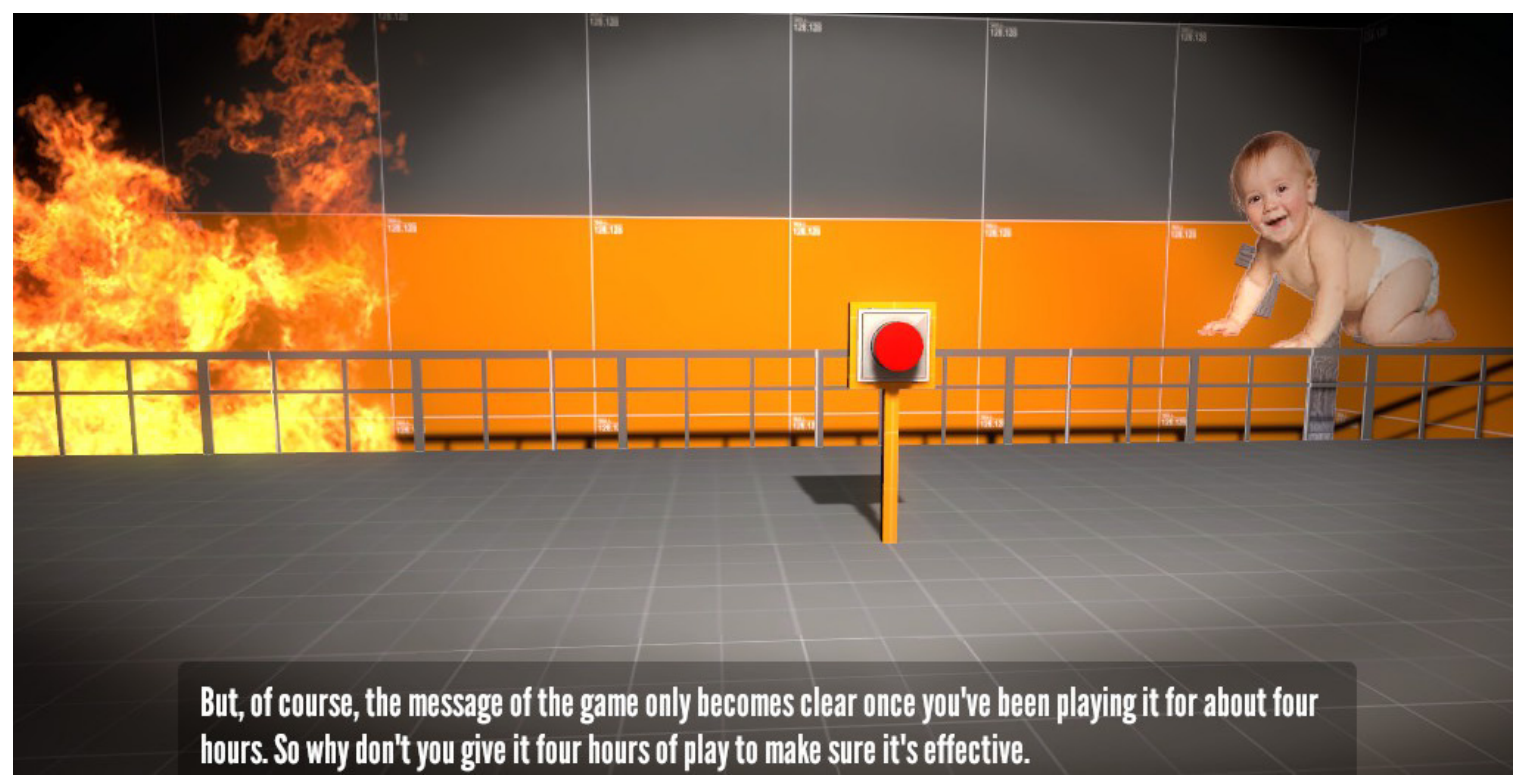

Fig. 7.1: screenshot of the game from Davey Wreden's The Stanley Parable (2013)

These particular endings to The Stanley Parable not only poke fun at art games, but also the players (and reviewers) who superficially criticize nonconformist games and assert the superiority of their agency over that of the developer (recall the lawsuit against Mass Effect 3 for its unsatisfying ending). This attitude is manifest in the actions of players who choose to diminish the role of the developer by resisting a game's intended narrative in order to shape the story according to their own tastes. These actions further echo Jodi Dean's concern about the decline of symbolic efficiency, such that now players devalue the author's work, and would rather tell their own stories through the developer's materials. This is not to suggest that authors should have final interpretive authority over their work (in other words, this is not a protest against Roland Barthes' "Death of the 
Author"), but rather that players are challenging the function of the author-developer in narrative games. In the face of recent trends toward greater customization, multilinear storytelling, and open worlds that allow players to craft their own narratives, Wreden's work suggests that by handing over the narrative direction to players in the name of "meaningful choices," developers lose the ability to create critical and socially important experiences. As Wreden states, "[y]ou have to critique the things that you love for them to be better. ... I wanted this to be kind of a slap to the face [for video game developers], to say, 'Hey, take a look at what you've been doing so far.' I wanted to ask the question "Why are we doing this?"” (qtd. in Schreier).

Wreden delivers this critique by giving players freedom over their interaction with the world, but as players continue to replay and interact with the system, their choices become increasingly meaningless. As Stanley, the player ends up like Dean's subject of communicative capitalism and "gets stuck doing the same thing over and over again" (Blog Theory 40); despite their willful disobedience, the player is never rewarded with the answer that they seek, they are left only with the culmination of their slightly-varied experiences. Even the game's achievements mock the player's drive to be rewarded for their activity. Achievements are typically virtual badges given to players for playing well or fulfilling specific tasks, but in The Stanley Parable, players must complete actions that are unrelated to typical game goals, such as "[p]lay The Stanley Parable for the entire duration of a Tuesday" or "Don't play The Stanley Parable for five years." The game itself does not end; it does not release the player from the experience: as the game's loading screen states, "the end is never the end is never the end." Instead, players must break the cycle and piece together an ending by analyzing its structure and narrative. 
Wreden's work demonstrates that games can be designed to disrupt the repetitive circulation of opinion to facilitate critical thinking. By putting players in opposition to the narrator, the game encourages players to break from its cycle of repetition, as without this break, the player is reduced to occupying the role of Stanley, an empty subject following and entering instructions into a computer.

The game uses a strategy similar to Sicart's approach to ethical game design. In Beyond Choices (2013), Sicart describes ethical gameplay as a juxtaposition between a work or object's affordances and its cultural meaning. Sicart offers the example of GIANTmicrobe's gonorrhea plush toy and its affordances of its design as similar to a teddy bear. The discordance between the plush toy and its form as a sexually transmitted infection creates "cognitive friction," which he describes as the frustrating experience when design works against our intuitions (93). Sicart argues that this friction can be utilized by designers to create meaning: "[d]esign choices can generate emotional experiences in the user by increasing cognitive friction. By extension, in the context of ethical gameplay design, cognitive friction can be used as a tool to create these kinds of experiences" (94). Although Sicart's work explicitly examines ethical and moral gameplay, it is readily applied to critical thinking more broadly. The Stanley Parable creates a similar tension in the player by juxtaposing the interactive affordances of games that create agency with the narrator, a figure who represents linearity and authorial control.

The game's unusual approach to agency questions not only whether players can exert agency in a world constructed by a developer, but whether players should become the authors of the games that they play. Wreden's work masterfully crafts a world where 
the player is able to explore freely, but the game's narrator anticipates every eventuality and mocks the player, simultaneously critiquing the very possibilities that it provides. In an interview, Wreden cites BioShock as an influence on his work, and states that he wanted to create a game that "broke player expectations, that delivered a familiar experience in a new way" (qtd. in Mattas). Wreden's game suggests that with the right approach to design, games can produce critical works that challenge players on multiple levels and encourage critical engagement.

While customization and player-driven narratives make for exciting games, these features are antithetical to sustained critical reflection. In BioShock, for instance, it is only by retroactively revoking the player's agency (revealing that their apparent decisions were not their own) that the game becomes compelling and challenges the player's assumptions about their role. These critical moments interrupt play (often in a very literal way) that jars the player into considering more deeply the events of the narrative. Games that cater to their players will struggle to create experiences that go beyond the circulation of affect and opinion in communicative capitalism, although there is no guarantee that a game designed for critical reflection will succeed; as Sicart states, these conditions can only "potentially [generate] moral reflection" (95). At the same time, it is important to recognize that not every game needs to live up to these expectations; after all, games are a source of entertainment. Yet, trends in the industry that drive publishers to privilege some kinds of games over others has created a market in which it is becoming increasingly risky to produce critical games. As Tom Bissell writes in his article "Does the Sneaker Have to Matter?," people in the industry are "really worried that the meaty, single-player narrative games we all love — like, say, BioShock Infinite - 
are soon to be an endangered species, at least for a little while. That seems to be the prevailing wisdom. It depresses me" (Bissell). This concern about the direction of games is further echoed by feminist critics that demand more diverse representations in games. The easiest way to quickly assuage antagonisms in the gaming community is to hand over control to players, so that, in a certain way, each side gets what it wants. However, as Adrienne Shaw points out, letting players create their own characters does nothing to challenge stereotypes and poor representation; as Shaw explains, avatar customization "makes the 'problem' of representation an issue only for those players willing to design their own avatars. ... The goal in increasing representation in games is not expanding customization options but rather making more games that reflect more modes of being in the world" (Shaw 143). While the games discussed thus far may not be the best examples of diverse representation, they provide a critical design model that Shaw would view as better suited to encouraging moments of critical reflection, in part because these experiences are not optional. Such criticisms of player-centric design, however, have been met with extreme reactions by those who align with groups like Gamergate, who see demands for increased representation as a kind of censorship of games and, paradoxically, attempt to sway the industry by censoring developers through harassment campaigns and claims that developers are conceding to "leftist agendas." 20

In an analogous way, both Bissell and Wreden argue that narrative-driven games like BioShock offer players a thought-provoking experience that cannot be replicated in games that privilege player agency above all else. In certain respects, this perspective is

${ }^{20}$ See for example Campbell (2016), Robertson (2017). 
comparable to Michel Chaouli's critique of hypertext fiction in "How Interactive Can Fiction Be?" (2005); "critical engagement," he argues, paradoxically depends on shutting down (or at least severely diminishing) the return channel of communication, for only when participants are released from the labor of constructing a text on a material or topological level (becoming "passive recipients" in that respect) can they become hermeneutically active and think their own thoughts about what they are reading, seeing, or hearing. (608) The relationship between critical engagement and agency, however, is not so simple. The players of games do not need to be put in a passive position by using, for example, cutscenes to incite critical reflection. It is not that choice and interactivity interfere with critical engagement, but rather that when given freedom, players might (consciously or unconsciously) self-impose limits on the kinds of stories that they will tell themselves. This state mimics the paradox of painful art; as Jesper Juul outlines in The Art of Failure (2013), “[p]eople do not seek out situations that arouse painful emotions," and yet "[p]eople seek out art that they know will arouse painful emotions" (37). Similarly, players will play games that address difficult situations and experiences, but they are unlikely to create these situations for themselves. Developers, however, can use design to facilitate critical reflection by introducing a choice that produces an unexpected outcome, or through cognitive friction by creating a moment where agency is temporarily disrupted. In these moments, the disruption of player agency introduces a new perspective to attempt to force reflection. While a theoretical experience of limitless player agency makes for a fascinating design problem to solve, there is value in experiences that are disruptive and not optional, as they evoke the same worker strategies 
that Dean describes in her critique of communicative capitalism. There is something that is lost when games are designed for trends and it is precisely this loss that Wreden attempts to pin down in his more recent work, The Beginner's Guide, which in many ways is a refined examination of the issues around authorship, art, and agency that Wreden addresses in The Stanley Parable. For many, the connection between the two games may not be apparent given their stylistic differences, but in the conclusion of The Beginner's Guide, Wreden signals this link by drawing a literal bridge between the two games, which the following section will address in detail.

\subsection{The Beginner's Guide to Critical Media Theory}

The Beginner's Guide is an unusual game in that it stages a scenario in which players are invited to analyze a collection of incomplete games by a developer named Coda, with the explicit aim of not only uncovering his intention, but also assessing his emotional state at the time of creating these unfinished games. As the trailer for the game explains:

Let's say you sit down at a stranger's computer. You start opening files and looking through stuff and eventually you come to a folder that just says "my work." So you open it, and you click on a random file and it's a video game. . . If you had to guess, what would you say you know about this person right now? ... Do you think this person is happy, are they unhappy? ... What puts them at ease? Do you think they're lonely? What's missing in their life? . . Let's unpack this person. Are they upset about something or fighting something? What do you think makes this person angry, what kinds of friends do they have, do they even 
enjoy making video games at all? Take all of these images and hold them in your mind, and now try to imagine, without ever having met this person, who they are. Okay, let's do it. Let's find out if you're right. (Everything Unlimited Ltd.) The creator of The Beginner's Guide, Davey Wreden, also plays the role of the narrator, who is responsible for guiding players through a series of disconnected and incomplete levels that are supposedly designed by his friend, Coda (a fictional figure). To distinguish between Wreden's authorial and fictional roles, I will use Davey to refer to his narrator function and Wreden to refer to his role as the developer of the game. As you play through The Beginner's Guide, Davey offers his own interpretations of Coda's work, and even solicits players to send him their own interpretations of the game to Wreden's personal email address. This is a surprising gesture, considering that the game had sold approximately 154,000 copies within its first four months. ${ }^{21}$

The appearance of each of the game's levels varies greatly. The first is a desert town, the second a spaceship, then a house in perpetual need of cleaning, a series of prisons, a lecture hall, and so on. While some levels are completed simply by walking a few short steps, others present unusual obstacles, such as suddenly limiting the speed of the player's character, or unnecessarily locking the player in a prison cell for an hour. When such disruptive events occur, Davey offers to remove the obstacle, and explains to the player how the original mechanics operated so that the player may continue to examine each of the levels without the frustration of the experience. In one instance, after releasing the player from Coda's prison, Davey recalls a disagreement that he had with Coda about the purpose of games; as Davey recounts,

${ }^{21}$ Source: Steam Spy steamspy.com/app/303210, accessed 16 February 2016. 
This is something he and I used to argue about a lot, you know, whether a game ought to actually be playable, whether it means anything if no one can get through it, and I would always defend that ... all this work goes into the game, why not make it playable and accessible? And so we just got into heated arguments over it, and there was one time that after one of these conversations he went home and a day or two later he sent me a zip file entitled "Playable Games," that was full of hundreds of individual games, each of which was just an empty box that you walked around in and nothing else. (The Beginner's Guide) For Davey, it is clear that if a game cannot be enjoyed by others (if it lacks a social function), it serves no purpose. When given hundreds of empty boxes, he continues to search for a meaning or some kind of connection outside of the game, even when it is clear that this particular work by Coda serves to demonstrate a kind of unplayable game. Instead of being motivated by audience reception, Coda views games as an artistic form that can be both deeply personal, and address important questions about the medium.

Continuing his commentary on Coda's games, Davey attempts to draw connections between the disparate levels. He theorizes a kind of progression that he believes signifies that Coda is becoming depressed, alienated, and anxious about his creative work. Players soon learn that Coda has stopped making games altogether. Concerned about his friend's well-being, Davey seeks to support and encourage Coda by sharing his work with others so that he can show Coda how much everyone likes his games. As Davey explains, "[i]f all of your anxieties are being channeled into your work, then if the work ever fails you have no backup and you're just going to crash" (The Beginner's Guide). By sharing Coda's work, Davey hopes that Coda will be inspired to 
continue making games.

Despite his efforts, however, Davey's attempts to help Coda fail, and his later games become increasingly dark and destructive. In one of the final levels of The Beginner's Guide, the player is given the task of literally destroying Coda's games using a typical gun from a first-person shooter — the gun acts as a kind of eraser that dissolves away the walls until only white space remains. After completing this level, Davey tells us that Coda disappeared for many months, until one day, he receives a new game called "The Tower," a game that Davey describes as one that "doesn't encourage thought or engagement, it doesn't ask anything of [players] except a lot of [their] time" (The Beginner's Guide). The Tower consists of three puzzles that cannot accurately be characterized as puzzles. The first is an invisible maze that jolts the player and sends them back to the start when they make a mistake. The second puzzle requires the player to enter a six-digit code into a number lock, but does not provide any clues to the solution of the combination. And finally, if the player succeeds in navigating the first two challenges, they are presented with a door that simply cannot be opened. Even if one were able to somehow traverse the maze and solve the code through trial and error (which would have approximately 3.6 million possible combinations), there is no way to pass through the final door. Thankfully, Davey removes these obstacles, like he did for the frustrating sections of the earlier levels, by providing a bridge over the invisible, electrified maze, revealing the combination to the lock, and removing the locked door by manipulating the game's code.

It is worth pointing out, even if it is a bit tangential, that the bridge that Davey creates to pass over the invisible maze uses a particular wall texture that is found in "The 
Baby Game" from The Stanley Parable (see figure 7.2). To reach this particular ending, the player must resist the established narrative by deliberately taking the opposite action encouraged by the narrator at each possible moment. The bridge that connects Wreden's two games signals an important critique that is concealed thus far in the narrative of The Beginner's Guide and alludes to an important shift that occurs in the tone of the game when the player passes through Coda's locked door. If The Stanley Parable playfully ridiculed players who opposed the established narrative of the game, The Beginner's Guide supposedly promotes the opposite by encouraging players to take control of its narrative by imposing their own interpretations of Coda's levels, and modifying the experience of his levels with the help of Davey. The act of diminishing the author's authority over a work echoes Dean's assertions about the decline of symbolic efficiency, such that now players demand that authors relinquish control over the events of the story so that they may create a narrative of their own.

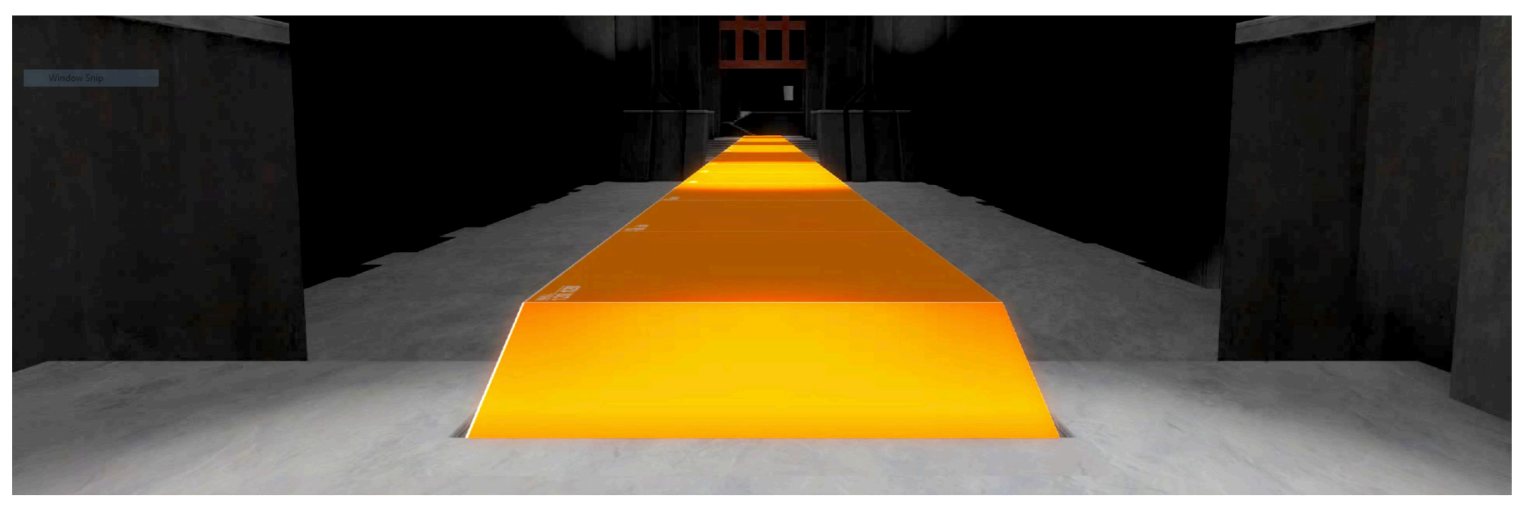

Fig. 7.2: screenshot of Davey Wreden's The Beginner's Guide (2016)

The inverse of games that concede to the player's demands are those that resist by using the medium in unexpected ways to disrupt the player's agency. Both "The Baby 
Game" in The Stanley Parable and Coda's level "The Tower" are characterized by repetitive actions that do not simply frustrate the player, but actively discourage play and are, in a certain way, unplayable. While The Stanley Parable's art game rewards, but laughs at the player's foolish persistence in pushing the button for four hours, the Tower does something very different and more serious. The puzzles found in the Tower are a direct response to the disagreement between Davey and Coda over whether or not games should be playable; but, Davey claims, the Tower's obstacles go beyond this disagreement: "the maze seems to despise the player for playing it at all." While Davey is correct, Coda's game does not simply resist play or the player, but disrupts the circular motion of drive by introducing an obstacle that makes it impossible to keep clicking. In the earlier levels, Coda's games attempt to enact a tactic analogous to the slow down, the same strategy that Dean attributes to the book as a site for critical media theory.

However, Davey deprives the player of the experience of frustration by removing the mechanics that inhibited the player's actions. Rather than accept delay and frustration as part of the game's intended experience, Davey modifies the mechanics to push beyond the game's boundaries and limitations. Davey further avoids the game's drive-disrupting effects by organizing its fragmented database of games into a chronological progression of levels, and establishes meaning across its disjointed pieces by constructing a coherent narrative around the apparent emotional decline of its creator. In this instance, contrary to Dean's claims, the suspension of narrative works against communicative capitalism. It is only when Davey is able to assemble the diverse levels into a coherent narrative about the game's creator that he is able to circulate and share Coda's work. 
For committing these transgressions against his work, Coda punishes Davey. Knowing that Davey will modify the code to remove the door in the Tower, Coda leaves a series of messages for him on the other side. When players pass through the removed door, they find themselves in an art gallery. The "art" that hangs on the gallery walls are messages for Davey that ask him to stop speaking to Coda, to stop sharing his work, and to stop tampering with his games. Coda's messages explain that he thought that if he added answers and meaning to his games that it would make Davey happy, but he has learned that it is never enough; as Coda writes, "You desperately need something and I cannot give it to you. I literally do not have it." After seeing these messages, Davey is clearly hurt, but still, he does the unthinkable: he shares Coda's games again. As Davey explains,

I am releasing this collection of your work, [it's] because I haven't been able to find any other way to reach you. I've tried everything. And... so a part of me has hope, that if I put this compilation out into the world, and if I put my name on it, that maybe enough people will play it so that it will find its way to you, so that I can tell you that ... I'm sorry. If I apologize ... will you start making games again? ... I know I did an awful thing, and I'm doing it again right now, I'm showing people your work, but I can't stop myself from doing it ... I'm an addict ... Just tell me what you want! . . please give me some of whatever it is that makes you complete. I want whatever that wholeness is that you summoned out of nothing and put into your work, you were complete in some way that I never was. 
Davey's plea for wholeness mirrors the struggle of Dean's "whatever beings." When symbolic efficiency has declined, identity no longer relies on social norms or symbolic identifications.

Symbolic identities, in contrast, provide stable characteristics to draw on through established norms and conventions (e.g. ideals related to one's social role as a mother or wife, husband or father), and regardless of whether one chooses to accept or reject such an identification, one's position relative to others remains well established. In communicative capitalism, however, identity has shifted to the imaginary realm where identity takes a fluid and flexible form through both the images that one selects to represent oneself, and the feedback and reflection of oneself through one's interactions with others. While imaginary identities may appear to be liberating in contrast to the much more restrictive symbolic identifications, identities established in the imaginary, as Dean argues, come at a cost:

Imaginary identities sustained by the promise and provision of enjoyment replace symbolic identities. ... These unique identities, though, are extremely vulnerable. The frame of reference that give them meaning and value are constantly shifting. Challenges to these identities can appear at any moment. Others' success, achievements, and capacities to enjoy too easily call our own into question. So while it may seem that the decline of symbolic efficiency ushers in a new era of freedom from rigid norms and expectations, the fluidity and adaptability of imaginary identities are accompanied by fragility and insecurity. (Blog Theory 57) 
Imaginary identities are established in relation to one's specular image: an image that is "simultaneously oneself and other" (Evans 190). As a result, identity is discordant and divides one's subjectivity between the self and its uncanny other in the specular image. One is always split between who one believes oneself to be and the incongruent image that is reflected back.

Coda's games come to serve as a point of identification for Davey. By showing Coda's games to other people, Davey gains recognition and validation (which he tries to share with Coda). However, without new games to reveal to others, Davey's identity breaks down. Because Coda and Davey's identities exist in different realms (Davey in the imaginary and Coda in the symbolic), Davey is unable to accept Coda's rejection of the notion that games should be playable. Imaginary identities are fragile and constantly need to be reasserted. Toward the conclusion of the game, Davey becomes aware that Coda's identity is divorced from validation, and for this reason he demands some of the wholeness that Coda puts into his work. Coda's identity is sustained in the act of creation and not the circulation of the work itself; his identity is independent of external validation. Therefore, Coda does not experience the same need to share his games, and even Davey's relationship to his work becomes a burden. Imaginary identities are not pinned down by the symbolic, but exist as the fleeting images on digital screens and one's ever-changing, personalized profile pages. An imaginary identity must be constantly reasserted and repeated, which is why "whatever beings" are driven by a compulsion to endlessly click, spreading more opinion, more affect, and more validation in likes, retweets, favourites, and upvotes. 
Dean argues that one result of the decline of symbolic efficiency and these fleeting and shifting imaginary identities represents "the dissolution of the boundary between fantasy and reality" (7). The breaking down of this barrier between what is real and what is fiction is precisely what causes Davey to misrecognize the difference between a game and its creator. Wreden cleverly reinforces the difficulties of this dissolution of fantasy and reality by doubling his presence in the game as both its creator and narrator. As one reviewer aptly notes: "[t]his game is like English class, where you super look into stuff and try to analyze it. It kind of makes you wonder, should I really appreciate those poems that they made me read?" (SwingPoynt). As any literature student will tell you, one should never conflate the narrator with the author. Davey, however, is unable to perceive the difference as he is helplessly captured by the drive of communicative capitalism. He cannot break from the repetition or jouissance that he experiences from circulating Coda's games. In Lacanian psychoanalysis, Dean explains, "desire and drive each designate a way that the subject relates to enjoyment. Desire is always a desire to desire, a desire that can never be filled, a desire for a jouissance that can never be attained. In contrast, drive attains jouissance in the repetitive process of not reaching it. Failure ... provides its own sort of success" (40). For Dean, the challenge for contemporary society is to disrupt the drive's hold on the subject, to break from the repetitive circles of communicative capitalism.

\subsection{Beyond Validation}

The epilogue of the game shows Davey struggling with what he now refers to as a disease: "More more more," he says, "[m]ore love, more praise, more people telling me 
that I'm good, always more" (The Beginner's Guide). Reflecting on the motivation of his actions, Davey explains, "[i]f I knew that my life depended on finding something to be driven by other than validation... what would that even be? It's strange, but the thought of not being driven by external validation is unthinkable, I actually cannot conceive of what that would be like!" (The Beginner's Guide) And then, suddenly, something clicks and Davey says that he has to go, he apologizes to the player, and then he leaves. Throughout this strange game, the player's presence has been feeding his drive for validation, and as Davey comes to this realization, he removes himself from the situation, leaving the player alone to figure out what exists not only beyond external validation, but beyond the circuits of drive. Players wander through the remainder of the level with no further contributions from the narrator. There is no fast-track to the end of this pause in action; instead, players must continue in silence and contemplate what they have witnessed. While exploring the abstract terrain, the player moves from darkness into the light as though to signal the player's arrival at a new perspective on his or her relationship to the game's themes of friendship, art, and validation.

On the 28th of February, 2016, several months after the game's release, Davey Wreden livestreamed himself watching a video of YouTube user Bowlingotter play The Beginner's Guide on Twitch. ${ }^{22}$ The husband and wife team featured in the video provide commentary on Wreden's game and its themes as they play through to its conclusion. In a similar fashion, Wreden adds his own commentary on their stream, noting their enthusiasm, curiosity, and the physical staging of their set. The viewers on Twitch

${ }^{22}$ The original recording of this livestream has not been archived, but one viewer has shared a screen-recording of the event here: youtu.be/aGAix9vHXW0 
interact with Wreden through the chat, eagerly asking him questions and expressing their excitement about his game. Wreden frequently reminds his viewers and those joining that he will not be speaking about The Beginner's Guide, and will only provide commentary on Bowlingotter's playthrough of his game. Quickly, the livestream becomes increasingly interactive as Wreden adds the livestreams of his viewers to his own, as many viewers had started their own livestreams of themselves streaming other viewers' livestreams of Wreden livestreaming Bowlingotter's video of the game (see figure 7.3). The bizarre and meta result of Wreden's livestream is both partially intentional and serendipitous.

Early into the stream, ${ }^{23}$ Wreden asks if Bowlingotter will reach a point at which they become aware of the parallels between their video and the game: "I assume there is a point where they go 'hey, isn't this weird that we're commenting about this game' ... where they officially acknowledge the weird [and] meta irony of them doing a video about a game about someone talking over someone's game" (Wreden). This is precisely why Wreden refuses to answer questions about The Beginner's Guide: his authority, as the author of the game, would impose some stable meaning on the work, much like Davey demands of Coda ("what do the three dots mean?"). This relationship of audience to author mimics the same problematic relationship that occurs in analysis when the analysand puts the analyst in the position of "the subject supposed to know," meaning that they believe that the analyst holds special knowledge that will unlock the meaning of their words. As Bruce Fink explains, "a patient who comes to the analyst in a highly suggestible state, sure that the analyst is the one with all the knowledge, is far less likely to realize that it is the patient who must engage in the serious analytic work of

${ }^{23}$ At approximately timestamp 0:5:20, see footnote 21 for a link to the video. 
association. Such a patient may be more inclined to briefly state his or her consciously formulated problem and await the analyst's indubitably brilliant solution" (Fink, Clinical Introduction 30). It is clear in The Beginner's Guide that Wreden intends for his players to do the analytic work, not of analyzing Coda, but the work as a whole and their relationship to it. He creates a similar effect in his livestream by withholding his supposed knowledge and attempts to show (rather than tell) his audience about the odd symbiotic relationship between game streamers and developers, and this new and strange economy of validation, where users share, comment, and capitalize on the work of each other. The income of Twitch livestreamers and YouTubers is based on their ability to accumulate likes, shares, and subscribers and this can translate into more sales for game developers. The parallels to communicative capitalism are apparent: the game economy is moving away from the consumption of a product, to the consumption of paratextual, extratextual, and metatextual content. 


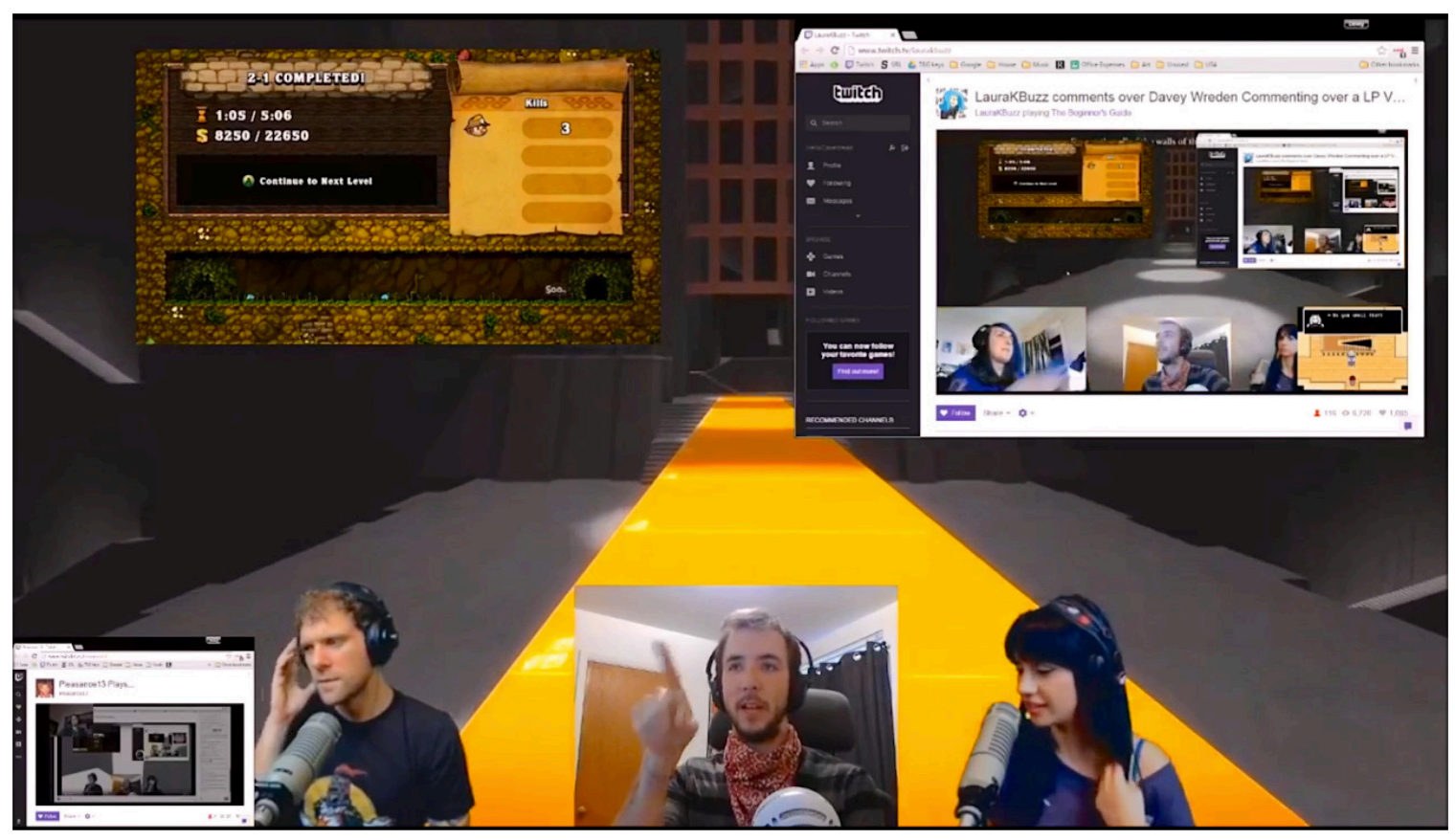

Fig. 7.3: screenshot from Davey Wreden's livestream on Twitch

Like Dean's conditions for critical media theory, The Beginner's Guide does not offer a happy solution to the drive's hold on the subject, nor does it offer hope or reassurance; instead, it invites the player to disengage from the circuits of communication, and encourages sustained critical thought about one's relationship to oneself and others, to question what motivates one's actions, and to rethink one's approach to narrative and art. In doing so, at least momentarily, the game disrupts drive by challenging players' expectations about what is possible in games. The Beginner's Guide succeeds as a kind of critical media theory not only because it incorporates sabotage through its disruptive and unplayable moments, but also because it reappropriates games as a medium for challenging the very hegemonic values and violent themes that games often perpetuate. If the reviews of The Beginner's Guide are representative of its reception more widely, it is clear that the game has caused its players 
to question their own relationship to the medium, their understanding of the relationship between creators and their art, and, more fundamentally, the cost of instant gratification and validation. The Beginner's Guide is a beginner's guide to a lot of things: to friendship, art, creativity, game design, and critical media theory, but its most important contribution to each of those areas is that it reinvigorates desire by forswearing validation, opinion, and affect, and instead encourages reflection and frustration. The Beginner's Guide offers a way to imagine other possible sites of critical media theory, while staying true to the conditions that Dean outlines without lapsing into overly optimistic or uncritical views of technology and popular culture. 


\section{Chapter Eight: The Shape of Games to Come}

\section{1 "Games Can Change the World"}

In part, the reason why the ludology vs. narratology debate continues to re-emerge is because players and game theorists believe that there is something that makes games distinct from other media as a critical art form, but what exactly this quality is remains debated. Galloway's countergaming, Wark's gamer theory, Flanagan's critical play, and Bogost's procedural rhetoric all examine the varied ways that games as interactive systems of representation and simulation offer persuasive and insightful commentary on contemporary society. Agency, identification, immersion, and interactivity fuel this fantasy that games are radically different than other media. As one looks more closely, however, these terms become decentred. Players' perceptions and experiences are often at odds with game design and the discourse used to describe player interaction: agency is illusory, identification is complex, and immersion and interactivity often interrupt one another. Moreover, these features are not exclusive to games; albeit rare, both print and film make use of interactive systems. As Bolter and Grusin explain, new media remediate older media, but this process does not necessitate the erasure of predecessors:

Games like Myst and Doom are desktop virtual reality applications, and, like immersive virtual reality, they aim to inspire in the player a feeling of presence.

On the other hand, like these computer games, immersive virtual reality also remediates both television and film: it depends on the conventions and associations of the first-person point of view or subjective camera. Sciencefiction writer Arthur C. Clarke has claimed that "Virtual Reality won't merely 
replace TV. It will eat it alive.” As a prediction of the success of this technology, Clarke is likely to be quite wrong, at least for the foreseeable future, but he is right in the sense that virtual reality remediates television (and film) by the strategy of incorporation. This strategy does not mean that virtual reality can obliterate the earlier visual point-of-view technologies; rather, it ensures that these technologies remain at least as reference points by which the immediacy of virtual reality is measured. (29)

Here, Bolter and Grusin demonstrate how our interaction with new media is informed by our experiences with earlier media. The similarities between VR and earlier media spur questions about what is new about "new media," and how (or if) more recent media improve on their predecessors. This creates a contentious relationship to older media, as the early days of any new medium are often fraught with criticisms that its content is somehow less authentic or of lesser value; e.g. "television will rot your brain." The impulse to counter such narratives is also responsible for the continued reemergence of the narratology vs. ludology debate. If, as Bogost argues, the narratives of games pale in comparison to those of print and film, we must answer why these narratives need to be told as games, or realize a vision for games that truly sets them apart from other media ("Better Without Stories").

Part of the answer is that media are imbued with all sorts of fantasies about technology and its promises and failures (e.g., the internet will change everything, or the internet will corrupt children). As Bolter and Grusin's work on remediation reveals, however, new media are always inextricably enmeshed with older media: new media are not as radically different from older media as these fantasies imply. In "Communicative 
Capitalism," Dean uses the example of the deeply held belief by music fans that "music can change the world" (62-63) to examine the way that technology is fetishized as a means of political action. There is an analogous belief about games and gaming: in her TED talk, "Gaming Can Make a Better World" (2010), Jane McGonigal states that her "goal for the next decade is to try to make it as easy to save the world in real life as it is to save the world in online games." ${ }^{24}$ McGonigal's own alternate reality projects, including World Without Oil (2007) and SuperBetter (2012), reflect this dream. Dean argues, however, not only that technology will not save the world, but that our fascination with new technologies and our belief that they are the solution to our problems are detrimental to our ability to take meaningful action:

The technological fetish covers over and sustains a lack on the part of the subject. That is to say, it protects the fantasy of an active, engaged subject by acting in the subject's stead. The technological fetish "is political" for us, enabling us to go about the rest of our lives relieved of the guilt that we might not be doing our part and secure in the belief that we are after all informed, engaged citizens.

(“Communicative Capitalism” 63)

Ian Parker similarly argues that the risk of technological fantasies is that they promise a resolution to our problems in the inevitable future: "[i]n science fiction we project fantasies into the future and are then caught in those fantasies both because they seem to confirm what has always been deeply true and hidden about human nature and destiny and because, we believe, we will eventually meet up with the fantasies when we arrive in

${ }^{24}$ See also: "Saving the Real World Together." Reality Is Broken: Why Games Make Us Better and How They Can Change the World, Penguin, 2011, pp. 297-344. 
the future" (Parker, Psychoanalytic Culture 203). Projecting our concerns into the future, however, delays our action in the present. An analogous mindset is responsible for the same failure to take meaningful action to address issues like climate change, whose consequences and solutions are viewed as problems for the future.

Games, however, reverse this logic. Rather than delaying action, players become incessantly active - saving the world again and again. It is this activity that McGonigal believes can be harnessed and put to work to achieve goals in the real world. McGonigal demonstrates this potential through her own alternate reality games, such as "Jane the Concussion Slayer," which she designed to facilitate her recovery from a concussion. She later developed this game into a more comprehensive version that was released to the public as SuperBetter. Alternate reality games in particular, she argues, have significant social benefits for the reason that they encourage players to engage with the real world: the less we fully participate in our everyday lives, the fewer opportunities we have to be happy. It's that plain and simple. The emotional and social rewards we really crave require active, enthusiastic, self-motivated participation. And helping players participate more fully in the moment, instead of trying to escape it or just get through it, is the signature hallmark of alternate reality projects. (Reality 124) McGonigal's work is unabashedly optimistic, but it is imperative to examine these ambitions and practices with a critical lens. As simulations, games allow players to try and retry solutions to problems in risk-free scenarios, which McGonigal believes will prepare players to create real world change.

This repetitious behaviour, however, is comparable to the incessant activity that is characteristic of interpassivity. As explained in Chapter 5, the term interpassivity 
describes a relationship with media that enables us to remain active and as a result “[deprives] us of our passivity” (Žižek, “Will you Laugh?”). In Žižek’s example, the VCR records television episodes so that we may continue working while satisfied that we can enjoy the content at a later time. This relationship with media becomes a problem when "later" never arrives. The incessant activity enabled by interpassivity is the source of the subject's capture in communicative capitalism: without moments of passivity, the subject has no time for sustained critical thought — one rarely has time to consume content, let alone consider or analyze it in depth. Consequently, one quickly reacts and responds in forms that contribute to communicative capitalism, for which one is rewarded with likes, retweets, favourites, and upvotes.

In games, players' actions and accomplishments are rewarded not just by the virtual world's characters, but also in the form of cultural capital as achievements and badges that are shared and displayed across social networks. Although games are often viewed as an unproductive use of time, it is no surprise that people turn to them when faced with unemployment or dissatisfaction at work. Games offer a form of enjoyment that can substitute the feeling of productivity that we hope to get from our jobs. As McGonigal says of gamers who play games for the equivalent of a half-time job, these players 'know when we're playing a game, that we're actually happier working hard than we are relaxing, or hanging out" ("Gaming Can Make a Better World"). Such a claim, however, raises concerns about the perpetuation of capitalist notions that value an individual based on their work ethic and productivity.

In Enjoying It: Candy Crush and Capitalism (2015), Alfie Bown takes an analogous, but more critical approach to the relationship between games, productivity, 
and enjoyment in his analysis of mobile games like Candy Crush (2012) and Football Manager (2006). Like McGonigal, Bown recognizes that these games offer players a sense of accomplishment; however, he argues that their function in society is not as innocuous as it appears. Contrary to conventional assumptions about productive and unproductive enjoyment, Bown argues that these two mobile games in particular demonstrate how games can supplement or augment the subject's position as a productive member of society. As a "distracting" game, Bown argues that the match-three game Candy Crush is not the time-waster that it is presumed to be. Instead, playing Candy Crush when one should be working instills a feeling of guilt that one is slacking on important work (which employees may not find all that satisfying or rewarding). This distraction is appealing, he argues, "because it simulates a feeling of satisfaction when we 'return to work"' (32). As a result, Candy Crush both facilitates productivity and assuages frustrations that might otherwise incite rebellion: this feeling of guilt creates a sense of responsibility for our situation.

In contrast to the distraction offered by Candy Crush, Bown characterizes the mobile version of Football Manager as an "investment" game, a term he uses to describe games that instil a sense of accomplishment and satisfaction in players. Football Manager achieves this aim by simulating the career of a team manager who must analyze statistics, make trades, and offer contracts to prospective football players. The skills and experience gained from playing these games, albeit rare, sometimes translates to real job offers. Some Football Manager fans have found employment in positions such as an analyst for the game (Stranger) and as the coach for a team in Azerbaijan (Garland). It is also worth noting that recruits of the United States Armed Forces have expressed interest 
in hiring gamers for their skills as drone operators (Drone 2014). These are, of course, exceptional cases and it may be a bit too early to include game achievements on one's curriculum vitae; however, as Bown notes, playing games can contribute to success in the workplace because these investment games supplement our desire for meaningful work through opportunities for learning and improvement:

Alienated in the workplace, we turn to a simulated feeling of career satisfaction which works to re-enforce the criteria we use to judge "success," only serving to exacerbate our sense of having more to achieve by creating an ideal of career success towards which we feel we should strive. [Football Manager] operates like Candy Crush, to prevent us from facing our dissatisfaction and thinking about it (which could at least potentially lead to organized opposition and revolt). (38) Contrary to analyses of games as sites of critical reflection, Bown investigates how, at least in his examples, games support and safeguard capitalism against critique by ensuring players remain active and satisfied. Bown's reading of agency and interactivity reveals that these mobile games are neither liberating nor subversive.

While Bown and McGonigal offer very different perspectives on the social and political significance of games, they share a common an emphasis on the active role of the player and the productive role of games in society; however, it is the nature of this productive activity that radically differentiates their approaches to games. Bown's work acts as a foil to McGonigal's optimistic view of games; as he writes,

I argue here that it is of political importance to realize that this enjoyment is anything but unproductive and in fact should be seen as particularly productive form of modern subjectivity. This is not meant in any positive way. Rather, we 
must see that there is an organized and organizing agenda behind the effects that these forms of apparently innocent enjoyment produce. (26)

Given Bown's assertions about the role of games in supporting hegemonic values, in the era of communicative capitalism, the most radical action might be a kind of passivity. It is imperative to first qualify that this usage differs from Dean's use of the term to describe the state of Agamben's "whatever being" in communicative capitalism. Drawing on Freud, Dean argues the whatever being is a passive subject; as she explains, they turn their attention from the content of language, from trying to communicate something, back to themselves as speaking. They shift from focusing on something outside or beyond themselves to turning back round upon themselves. In his discussion of the drive as precisely this turning round upon the self, Freud views it as a change from activity to passivity. The active aim, to say something, is replaced by the passive aim, to have said. Whatever beings are passive, then, because they are subjects of drive. (85)

This view of the subject of communicative capitalism is a seemingly contradictory but not incongruous statement. The subject is passive in the sense that they are caught up in the circular movement of the drive, but this state demands constant activity to reaffirm one's identity in the absence of symbolic authority (much as Davey demands of Coda in The Beginner's Guide). To suggest that passivity has the potential to disrupt this subject position does not conform to Dean's use of these terms, but rather should be taken in the context of the subject's interpassive relationship to media and the activity that it facilitates. In other words, the subject's capture in the drive must be interrupted not by some action, but by a disruption that ceases its circular movement. 
Such an intervention, Dean argues, would mimic the tactics of workers' labour struggles. As discussed in Chapter 7, her analysis of the book as a site of resistance is promising, but could be developed further. The book as a medium does not necessarily create the conditions for fissures in communicative capitalism, but, in a certain way, it does require them for its production and consumption — it requires sustained critical thought. The book, however, is not outside of capitalism. Like all media, it is an object designed to be purchased and eventually discarded for more recent editions and additions. As Bown writes, "the enjoyment of critical theory indicates that the material is often consumed and enjoyed as a product in precisely the way the theories in question [Lyotard and Deleuze] would oppose, and in precisely the way the structures that they attempt to resist would command" (13). As an example, he describes social media posts that depict readers enjoying works by Deleuze at the beach (19). Bown's criticism necessarily applies to Dean's account of the book; as Bown notes, "[w]hilst critical theory can be radically transformative, it is often enjoyed far more 'responsibly' than we think" (24). Despite the time and effort spent consuming these works, he argues, they are rarely put into practice.

"Enjoying Lyotard and Deleuze," and, I would add, Dean, "as critiques of capitalism, works on one level against the conditions of our system and creates new languages for resistance, but it can also completely conform to the prescribed enjoyments of our moment and fall into re-enforcing the order it seeks to dislodge" (Bown 21). Critique becomes commodified as capitalism reappropriates the aesthetics and methods of resistance used by the working class for its own purposes - for example, by commercializing dissent in the form of printing rebellious slogans on t-shirts, mass 
producing the iconic Guy Fawkes masks used by Anonymous, or by co-opting protest movements to advertise Pepsi products. ${ }^{25}$ As Mark Fisher (2009) elaborates, "Witness, for instance, the establishment of settled 'alternative' or 'independent' cultural zones, which endlessly repeat older gestures of rebellion and contestation as if for the first time. 'Alternative' and 'independent' don't designate something outside mainstream culture; rather, they are styles, in fact the dominant styles, within the mainstream" (9). As such, these examples serve to neutralize and dilute dissent.

In Gamer Theory, Wark offers a similar take on critical theory through his analysis of the gamification of academia:

Even critical theory, which once took its distance from damaged life, becomes another game. Apply to top-ranked schools.... Pick a rising subfield. Prove your abilities. Get yourself published. Get some grants. Get a job. ... You won! Now you can play! Now you can do what you secretly wanted to all those years ago ... Only now you can't remember. ... Your critical theory became hypocritical theory. (017)

Although Dean does not use this terminology, it is precisely this gamification of academia that enables communicative capitalism to capture critique. The notion of applying limits, time-pressure, achievements, and goals to academic endeavours certainly does evoke game-like mechanics.

Unsurprisingly, designing critical games is similarly problematic. Generally, games are meant to draw in players to increase sales and this aim is often at odds with

\footnotetext{
${ }^{25}$ This Pepsi advertisement (2017) featuring Kendall Jenner was pulled after facing
} backlash on social media for trivializing political resistance. 
critiques of capitalism. Further, as a source of entertainment, players will not necessarily enjoy games through a critical lens: as Sicart notes in his discussion of agency and ethics, there is no guarantee that players will interpret a game's meaning as developers intended (110). This critique of the commodification of critical media, however, does not deprive Dean's theory of its usefulness, as she is well aware of the fetishization of media objects. Although she argues that the book is best able to resist communicative capitalism, its true strength is not the medium itself, but its use of particular tactics and strategies (like the slow-down) that resist and disrupt the compulsion of the drive. Like books, games also take significant time to create and play, but their value as critical works comes from these same strategies that work against conventions and expectations. Delaying the player's pursuit of game goals may incite reflection about the game's events and their meaning, but such a strategy can be antithetical to the success of the game, not only in terms of its financial success, but also its ability to reach players and maintain their engagement. Walden, A Game (2017), based on Henry David Thoreau's famous novel, might be interesting as an object of analysis, but players may not find it as exciting as saving the world from aliens in games like $X$-COM (1994-2016), and this is precisely the point. It requires a certain amount of privilege to be able to create critical games - the kind of privilege that Allison Harvey notes that marginalized communities lack. That critical games should be marketable to players is inherently problematic, and the challenge of making such games is exacerbated by audiences that view games as asymmetrical to other artistic media. While not true of all players, it is evident from surveying game reviews that there is a general resentment to paying a sum of money (comparable to the cost of a film or book) for experimental games. A number of reviews for The Beginner's 
Guide, for instance, were disappointed by its price (\$10.99 CAD) and lack of game-like qualities; as one reviewer wrote, "It is literally just a story, nothing more at all. Definitely not worth more than $\$ 2$ " (poochyena). That a story could be seen as worth so little is itself troubling, but this issue not only speaks to tropes about "starving artists," but also to industry trends that are driven by player-centric experiences and players' expectations for what constitutes a game (e.g. features such as agency, customization, multilinearity, multiplayer).

It seems that the fear that one's favourite artists might "sell out" has gone out of style. Subcultural audiences are now more concerned about experience than a theoretical loss of authentic artistic expression when art meets popular culture. In this phase of capitalism, the author or artist rarely stands out as a trusted authority on political issues; instead, they are cognitive labourers in the business of content creation. Outside of academic discussions, there has been an especially contentious battle taking place over whether games are purely entertainment or whether they have a greater artistic purpose. In the years following Gamergate (2014 onward), games that suggest political messages have faced backlash from particularly vocal but likely small audiences who criticize games that cater to leftist values like political correctness and inclusivity, and demand that developers "make games great again" by returning to a time when games were not politicized.

Perhaps one of the most apt and absurd examples of this controversy occurred in the weeks leading up to the release of Wolfenstein II: The New Colossus (2017), when its developers faced a slew of criticism from fans who were angered by the game's anti-Nazi stance, which they argued aimed to capitalize on "liberal leftist propaganda" in the wake 
of the Trump presidency (Marcin). Responding to these criticisms, the game's social media marketers doubled-down on its position and released a video that declared "There is only one side." That this series in particular was subject to this kind of criticism is somewhat surprising, given that since its first iteration, Castle Wolfenstein (1981), these games have been about defeating fascism: there is no depoliticized "golden age of gaming" to which Wolfenstein could return. This example highlights a number of the strange effects that communicative capitalism has had on the politics of aesthetics, including the introduction of new challenges for the critical role of art in a society where communication is fragmented and flattened.

\subsection{The Subject of Communicative Capitalism}

Fundamentally, this concern about the state of art is an analogous of the longstanding problem of how to critique ideology from within its structure. In $O n$ the Reproduction of Capitalism (1971), Louis Althusser describes the process of interpellation whereby individuals are captured as subjects of ideology. As a result of this capture, he argues, it is only from within ideology that subjects can critique their situation; ideology has no outside (192). What follows does not propose a resolution to the dilemma created by launching a critique of communicative capitalism from within, but instead draws on more recent work by Dean, Bown, and Fisher to theorize how games as a site of critical media theory fit into the current political landscape.

In "Videogames of the Oppressed," Frasca proposes that the power of games as a critical tool comes from their ability to simulate alternate possibilities. Borrowing from the work of Augusto Boal, Bertolt Brecht, and Paulo Freire, Frasca imagines a kind of 
subversive game that interrupts player immersion to facilitate critical thinking about alternate solutions to a problem or situation; as he explains, "the goal of these games is not to find appropriate solutions, but rather serve to trigger discussions" (90). Frasca, however, is cautious about the transformative power of such a game, as he adds that "[n]either art not [sic] games can change reality, but [he does] believe that they can encourage people to question it and to envision possible changes" (93). Although this vision is encouraging, it risks mobilizing the very tactic of communicative capitalism that proliferates opinion in order to dilute critique. As Dean argues, what is needed to overcome the obstacles of communicative capitalism is a strategy that reinstates desire and brings meaning to action.

Despite his earlier reservations about the subversive potential of critical media in Enjoying It, in The Playstation Dreamworld (2018), Bown expands his Lacanian approach to games to theorize a more optimistic account of games as critical works. For Bown, "the world of videogames can only be fully understood via the analysis of French psychoanalyst Jacques Lacan" (3), and it is through this lens that he is able to develop a more concrete understanding of the player's relationship to games. Using this approach, Bown argues that games are uniquely positioned to resituate the subject in relation to desire, a strategy that could be leveraged by the Left to counter alt-right movements, which themselves have been very active in garnering support in sectors of the gaming community. As Bown writes,

Could we conceive of a video game which aims at reprogramming desire against the fascist, corporate, and capitalist tendencies found in videogames in general, or would the only 'morally ethical' game be the kind which denaturalized desire, 
showing our wishes to be not natural but the naturalized wishes of the other? This is a question about the politics of subversion in a much wider sense, since embracing this possibility would involve an admission that it is necessary not only to deconstruct existing ideological assumptions but to construct new ones, operating consciously to manipulate the emotions of others. It may be time for the left to accept this necessity. (78)

While Bown does not draw on Dean's theory of communicative capitalism, his diagnosis of current fascist and capitalist ideologies arrives at a similar conclusion and a call for the left to take action. Fundamentally, the subject's capture in communicative capitalism is the result of a disruption to the normal circuits of desire; as Dean explains, "[c]ommunicative capitalism thrives not because of unceasing or insatiable desires but in and as the repetitive intensity of drive" (Blog Theory 30). Bown theorizes that games can serve as a site to subvert ideology by revealing or configuring a new relationship to desire. Games make such an aim possible because of their capacity to reveal the underlying algorithms that structure our experiences (79); such a hypothetical game would reveal our capture and replace fascist and capitalist ideologies with leftist values. Bown points to the popularity of survivalist games as fuelling individualist ideologies about meritocracy and supremacy to argue that these "dystopian futures promote the idea that only capitalism separates us from a barren wasteland" (41). While Bown points to games like Papers, Please as an example of a subversive work, his theory is more strongly rooted in enjoyment; as he explains, "while the majority of producers and the majority of gamers approach the game with either conformist or apolitical attitudes, the enjoyment produced between subject and game can nevertheless be politically 
subversive. If this subversive potential of the dreamworld can be harnessed, it can be used to reach a wide and often initially unreceptive audience" (115). How exactly this dreamworld might be harnessed, however, is a bit unclear. His conclusion speaks to the subversive gamer rather than the developer, but one has little control over one's enjoyment of a game and so how one might mobilize this enjoyment is unresolved.

It is also necessary to address the concern that he alludes to in his suggestion that this form of manipulation may not be a tactic that is welcomed by the left. I would add that it is also a tactic that is problematic from a Lacanian perspective as well. The idea that it is necessary to manipulate and "reprogram desire" rather than facilitate critical thinking is not only unsettling, but also unethical in the context of Lacan's clinical work. The process of deconstructing one's relationship to desire is a crucial stage in the process of analysis, but the goal in so doing is not to remove or replace the cause of desire (objet petit a), but rather to redefine one's relationship to it (Fink, Clinical Introduction 206). Desire is central to Lacan's ethics, and dissolving the subject's relationship to desire can have severe consequences since it is the structuring principle that sustains one's worldview. For Lacan, ethics is a question of whether or not one has acted in conformity with one's desire (314), and that, "from an analytical point of view, the only thing of which one can be guilty is of having given ground relative to one's desire" (319). Therefore, destroying another's desire prevents the fulfilment of this pursuit and is unethical. Removing the very thing that constitutes the subject in the symbolic will cause at best melancholia and at worst a psychotic break.

Confronting the real of one's desire can cause what Lacan refers to as a passage $\grave{a}$ l'acte, a breaking point that functions as a self-defence against the anxiety that arises 
from this knowledge about one's desire. The term describes an impulsive act that serves as "an exit from the symbolic network" (137); as an example, Evans discusses one of Freud's cases where a woman survived a sudden leap from a bridge (137). In a proper analytic setting, the analyst works with the analysand to deconstruct desire to resolve the internal conflict that has brought the analysand to seek help. With the support of the analyst, the analysand is able to progress toward the end of analysis; however, without this guidance, the subject could undergo a passage à l'acte as a violent outburst or flee from the knowledge and reconstitute their identity in the imaginary.

Applying these concepts to Chris Noonan's film Babe (1995), Anna Shane illustrates how the end of analysis corresponds to a shift from metonymy to metaphor. In the film, Babe comes to realize that the purpose of his life is death; as a pig, he is viewed as "the sum of all his parts - chops, ham, bacon... all parts have exchange value." This realization sends Babe, who had believed he was a beloved pet, into a depression. Shane argues that "Babe's problem is to find a way to enjoy his life, even though it doesn't mean what he thought it did." Through the love of the farmer, however, Babe is able to accept his destiny which transforms him; he becomes "no longer a pig of metonymy, where each part of him has value and can be exchanged, but a pig of endless metaphor, who may be whatever he likes, as long as he knows he isn't" (Shane).

Similarly, one might examine the subject of communicative capitalism. According to Dean, "[t]he intensification of capitalism amplifies pressures on and for the individual. These pressures are political: the individual is called on to express her opinion, speak for herself, get involved. She is told that she, all by herself, can make a difference" (Crowds and Party). However, should the subject become sceptical of the 
impact of her efforts, she may come to believe that despite her dedication and enthusiastic participation, on a grand scale, her contributions are insignificant and irrelevant. This is likely a common insight that one invariably, but only fleetingly, acknowledges. For instance, one may become disillusioned with voting, perhaps believing that an external agency is meddling with the results, but then turn to other platforms that they view as free from such interference to share their opinions. More broadly, this experience is the result of a conflict between an ideology that constructs citizens as individuals and democracy as a system that is ruled by a majority. As Žižek avers " "they know that, in their activity, they are following an illusion, but still, they are doing it'. For example, they know that their idea of Freedom is masking a particular form of exploitation, but they still continue to follow this idea of Freedom" (Sublime Object 30). For Žižek, we act contrary to our knowledge about exploitation because our behaviour is tied to ideology — to the fantasy that structures reality. As in the example of the individual, the burden of one's supposed importance is a fantasy that is easier to accept than a tragic reality where one is utterly irrelevant.

Should one dwell on the conflict between one's scepticism and one's desire for individuality and freedom, one may begin to call into question one's value and fall into a deep despair. It is at this point that the subject finds itself in trouble, and to resolve this situation, the subject must either seek help from a professional clinician, or risk undergoing a passage à l'acte. In this example, the most likely result is that the subject will reconstitute their identity in the imaginary by joining a group that validates their contributions and makes their efforts feel meaningful and impactful, thereby contradicting the knowledge that had caused distress. This result may provide an insight 
into the surge of participation in fascist movements. Reconstituting one's identity in the imaginary, however, creates a repetitious cycle where a fragile ego must be continually reinforced or else the process will begin again. In The Beginner's Guide, Davey's character represents such an ego, as he relies on Coda's work to sustain his identity. Ideally, however, the subject would confront this knowledge in analysis.

For Babe, the solution to his crisis is to move beyond metonymy to metaphor; in other words, "the passage of the signifier into the signified, the creation of a new signified" (Evans 112). For the subject of communicative capitalism, this process would entail that the subject renounce their identity as an individual in favour of the signification of the metaphor, which is, in Dean's work, the collective. Instead of a subject fragmented by various (and often conflicting) identifications, the subject of endless metaphor "may be whatever [it] likes, as long as [it] knows [it] isn't" (Shane). By moving away from privileging the individual, it frees the subject from the weight of the associated responsibility. In Crowds and Party (2016), Dean critiques the individual and theorizes the potential of the crowd against its typically negative association as the mob:

Rather than a locus for creativity, difference, agency, and responsibility, the individual is the overburdened remainder of dismantled institutions and solidarities. Commanded individuality obscures individual incapacity even as it implies the contradictions barely congealed in the individual form. At the same time, these commands and incapacities attest to another force, the power of collectivity that manifests in crowds. 
While Dean does not present her theory in terms that address the end of analysis, it is clear that what she envisions is an analogous shift away from the signifier of the individual to the metaphor offered by the crowd. As Shane elaborates,

This is the end of analysis. This is what you find out when you cross your phantasm, because a phantasm is, after all, a signifier. A privileged signifier, with ties in the imaginary, but still a signifier, covering the real. In recognizing this knowledge, the subject assumes his destiny, which actually amounts to the same thing for all subjects. We are all faced with lack of meaning anywhere outside the signifier, which merely refers to other signifiers, not to some great and guaranteeing truth. (Shane)

The aim of the end of analysis is not an end in itself, but instead serves as a step in the preparation for the analysand to become an analyst (Evans 54): "[t]he end of analysis entails a shift in the analysand's transference, from the figure of the analyst, via the real, onto the cause of psychoanalysis itself - meaning that every analysis is, retroactively, a training analysis" (Aikatsurama). Unlike other forms of psychotherapy, the end goal of psychoanalysis is not happiness; as Lacan states, "[t]here's absolutely no reason why we should make ourselves the guarantors of the bourgeois dream" (Ethics of Psychoanalysis, 303). Instead, the end of analysis aims to "confront the one who undergoes it with the reality of the human condition" (303), an experience that brings anguish and distress with knowledge (304). Bruce Fink argues that this "is the stage of Lacan's work where desire is endowed with a certain utopian edge: it can take us where we want to go- that is, beyond neurosis" (207). 
Yet, as Fink states here, this approach is designed to attend to neurotic clinical structures. Although it is not explicit in Bown's formulation of the critical potential of games, his analysis presumes a neurotic or perverse clinical psychic structure - given the reliance of his approach on the centrality of desire. A relationship to desire, however, is not a present across all three of Lacan's clinical structures. It is absent in psychosis, and as Dean argues, contemporary society is more accurately characterized by this more troubling psychical structure:

If narcissistic culture is characterized by the dislodging of symbolic authority, psychotic culture is characterized by its foreclosure. In brief, Lacan defines psychosis in terms of the foreclosure of the Name-of-the-Father or master signifier. That the master signifier is foreclosed means that it does not stabilize meaning; the signifying chain lacks an anchor that can hold it together. The generalized loss of symbolic power impacts the subject such that he feels this now-missing authority to be all the closer, more powerful, and intrusive. In a psychotic culture, then, mistrust is pervasive, all-consuming. (Crowds and Party).

The absence of symbolic efficiency is an essential characteristic of psychosis. When the symbolic father is foreclosed, it is indicative that one has not properly passed through the stages of subjectification; the subject of communicative capitalism therefore is not a subject. In psychosis, there is "no possibility for the existence of a subject" (Fink, Between Language 74), as the analysand has not undergone the fundamental split that establishes the subject in relation to objet petit a (the cause of desire). As a result, the psychotic has no relationship to desire: “The 'dialectic of desire' has no place. There is 
no properly human desire at all in psychosis. Where the structure of language is missing, desire too is missing" (Fink, Clinical Introduction 101).

To treat psychosis in an analytic setting, the analyst cannot simply instate a paternal metaphor to create desire; this relationship can not be created later in life: "once a psychotic, always a psychotic" (82). The analyst's treatment of psychosis is a matter of managing symptoms and psychotic episodes (e.g., passage à l'acte) rather than a cure. Analysis does not prepare psychotics to be their own analyst as the end of analysis does for the neurotic. The psychotic learns to function in society by relying on imaginary relations, meaning that for the most part their behaviour is a kind of mimicry. As detailed in Fink's account of a documented clinical case of psychosis, attempts to interpret the psychotic's words or impose symbolic meaning on their world can fuel paranoia and trigger a psychotic break. What such a diagnosis means for society, however, is yet to be determined, but one can glean from Lacan's speculations that the prognosis is poor: Lacan does not assert that the father should be propped up in our society. Rather, he issues a warning: to reject the father's role, to undermine the father's current symbolic function, will lead to no good; its consequences are likely to be worse than those of the father function itself, increasing the incidence of psychosis. This is one of the things Lacan had in mind when, in 1971, he entitled Seminar XIX “... ou pire" : “. . or Worse"), one of the possible elided words being père ('father'). If we view the father as the lesser of two evils, to reject the father is to opt for the worse.

Lacan's challenge to discourses that encourage the elimination of the paternal function would run something like this: "Can something like the paternal 
metaphor-providing the fundamental link between signifier and signified, between language and meaning — be instated without the father as symbolic function? If so, how? If not, is there some other way to introduce an outsidethat is, to triangulate the mother-child relationship and stave off psychosis? How can this be done without relying on the symbolic order and its ability to intercede in the imaginary, the world of rivalry and war? Doesn't one sex have to play the part of symbolic representative?"

Unless some other way of achieving the same effect is found—Lacan's work would seem to suggest — the practices that stem from such discourses run the risk of increasing the incidence of psychosis. (Fink, Clinical Introduction 111)

Fundamental to the treatment of psychosis is the need to prop up identity by constructing a narrative that gives meaning in a way that sustains rather than examines the psychotic's worldview. This approach, however, is wholly incompatible with the treatment of neurosis.

In his discussion of psychosis, Bruce Fink shares the case history of a man named Roger who was treated for this condition. Over the course of his treatment, Roger sees two therapists. His first therapist fails to properly diagnose Roger and attempts to provide him with an interpretation of the dreams and writings that he brings to analysis. As a source of knowledge, the analyst attempts to intervene as a symbolic authority, as "the Other: the place or locus in which meaning is determined" (Clinical Introduction, 104). However, in psychosis, no such position exists, and as a result, rather than creating a breakthrough for his analysand, this intervention triggers Roger's psychosis and he begins to experience delusions (105). The psychotic lacks an anchoring point that enables 
the movement between signifier and signified (107), and for this reason the analyst cannot simply introduce a paternal metaphor or symbolic authority. In the treatment of psychosis, the analyst "must focus all his efforts on the imaginary register that is there and operative, to make it as sturdy and solid as possible" (108). The goal here is not to deconstruct and reveal unconscious meaning as one would in dealing with neurosis, but instead to

construct an understanding, edit a world of meaning that allows the individual to live and find a place for him- or herself. ... the therapist must encourage such meaning-making activity because the ego is all one can work with: the therapist must build up a sense of self in the psychotic that defines who the psychotic is and what his or her place is in the world. (108-9)

Through this work, the analyst creates what Lacan refers to as a "delusional metaphor," a narrative that serves a similar meaning-making function as the paternal metaphor, "the basis of which the psychotic establishes the meaning of the world and everything in it" (109).

Both clinical approaches to neurosis and psychosis are fundamentally a strategy to rewrite the analysand's narrative. In cases of neurosis, the analyst reveals to the analysand a world of unconscious meaning that, in a successful analysis, leads "the analysand to articulate the truth about his desire" (Evans 53) and repositions the subject in relation to the Other. In the treatment of psychosis, the analyst weaves a narrative out of the psychotic's words to provide their world with stability. Analysis offers a kind of agency over our desires and our anxieties that, although illusory, has powerful effects. In his narratological interpretation of psychoanalytic theory, Ricœur explains that, 
the story of a life grows out of stories that have not been recounted and that have been repressed in the direction of actual stories which the subject could take charge of and consider to be constitutive of his personal identity. It is the quest of personal identity which assures the continuity between the potential or virtual story and the explicit story for which we assume responsibility. (30)

Though we cannot change the past, psychoanalysis works to liberate the subject to become, as he writes, "the narrator and the hero of our own story, without actually becoming the author of our own life" (32). Narrative is essential to the analytic process as both a point of access to conscious and unconscious content and as a tool to reach the end of analysis.

Yet, it is not simply narrative, but the intervention of the analyst, a third term, that is introduced between subject and Other that gives narrative its analytic power. Without this intervention, one's understanding and interpretation would be impeded by defence mechanisms and paranoia. As Freud states, "in self-analysis the danger of incompleteness is particularly great. One is too soon satisfied with a part explanation, behind which resistance may easily be keeping back something that is more important perhaps" ("The Subtleties of a Faulty Action" 4820). The analyst is able to challenge and push beyond these boundaries. This is precisely why I raise the question concerning what kinds of stories players would tell themselves if given the freedom: without the intervention of a third term — such as an author-like figure — players are unlikely, or as Freud might argue, unable, to engage in an in-depth critical analysis. Ignoring the technical limitations that freedom presents, such a game would, in theory, reduce play to the level of fantasy. A critical game, therefore, must challenge fantasy in some way. However, in order to 
imagine the shape that such critical games would take, it is essential to first address the conflict that arises between the psychical structures in the application of this psychoanalytic approach to society.

\subsection{Conclusion}

While generally individuals are more likely to be neurotic, if Dean's diagnosis of contemporary society is correct, then this psychoanalytic approach to critical media theory reveals a deadlock concerning whether it should operate at the level of the individual or attend to a broader cultural disorder. Each requires fundamentally opposing responses and choosing the wrong approach will re-entrench the neurotic in their symptoms or trigger a psychotic episode in the psychotic. What is at stake in this approach is the ability to incite a form of critique that goes beyond the kind of manipulation that Bown describes in The Playstation Dreamworld. The preferable outcome of this conflict is an application that mimics the end of analysis, which offers a utopian resolution that would see players become analysts. In contrast, a critical media theory based on psychosis would entail managing the symptoms of Dean's psychotic culture, rather than a progression toward an endpoint. Ultimately, this conflict is a question about whether to attend to social concerns at the level of the universal or the particular. From a psychoanalytic perspective, there is no intermediary approach that can benefit both positions because their symptoms require opposing responses.

Dean's political theory of the crowd requires a shift from the individual to the collective, a strategy that is analogous to the subject's movement from metonymy to metaphor at the end of analysis. In the crowd, one is free from the burden of individual 
responsibility, which, like her critique of identity politics, suggests a subject can take on any number of identities while simultaneously acknowledging that they are constructed and divisive. For Dean, identity politics disempowers political movements. It is not that the concerns of these groups are in some way secondary; the issues raised by feminism, for example, must be addressed. Yet, identifying as a woman has historically been a criteria for excluding women from political participation (e.g. voting). Instead, collectivity and solidarity are best suited to respond to these issues by addressing them at the level of the relations of production and economics; a crowd, she writes "provides an opportunity for the emergence of a political subject" (Dean, Crowds and Party). As she explains, this theory is developed through her inversion of Althusser's notion of interpellation, which she rewrites as a theory of individuation:

Confronting a crowd or mass, the ideological hail fragments it into singular elements. Montag notes the specific resonance of Althusser's use of the term "interpellate" in the context of the protests and demonstrations of the 1960s. One would be stopped by the police "and therefore singled out from the crowd or singularized in relation to a background." What was a crowd becomes so many separable individuals. (Crowds and Party).

As a result of this individuation, the crowd loses its power. Dean argues that the party, specifically the Communist Party, can harness the energy of the crowd after it disperses and carry its momentum forward to effect political change.

A corresponding approach to critical games based on the end of analysis would require the same shift from metonymy to metaphor. In some ways, this shift resembles that which takes place in The Beginner's Guide when the player is encouraged in subtle 
ways to move beyond the game's initial goal of analyzing Coda to a more broad consideration of games and art that aims to turns players into critical analysts. The linear narrative directs players to a singular outcome where it introduces Coda as a kind of third term that disrupts Davey's narrative, and in so doing, it gradually moves beyond the particular (Coda) to the universal (games). Wreden's work provides a strong example of a critical game that attends to neurotic symptoms, but as such it will not resonate with all players. Those who have a psychotic psychical structure would likely respond negatively to this game, perhaps because Davey's character too closely resembles those who are similarly beholden to the imaginary. Such negative responses are manifest in those players who fail to see The Beginner's Guide as metaphoric, and conflate author and narrator, believing that Wreden has stolen and sold Coda's work, or those who have a more paranoiac response that involves Wreden manipulating players for financial gain. ${ }^{26}$

It is likely that the defensive reaction of the Gamergate movement could be attributed to a similar psychotic response to the imposition of meaning by game studies and feminist scholars — much like how Roger, Fink's case study, became delusional when too much meaning was given to his words. As game criticism moved into mainstream and was featured in game journalism and on YouTube channels, some gamers, rather than taking an interest in the work, felt that games were being taken too seriously, and that too much meaning was being applied to something they saw as just entertainment. This confrontation led to a distrust of media critics, and, consequently, the emergence of a community founded on a paranoid belief that games were under attack. In

${ }^{26}$ A quick overview of the game's reviews on Steam will reveal numerous examples of such responses, but it would be irresponsible to identify specific examples as revealing a psychotic psychical structure. 
Lacanian terms, they fled from the knowledge of the symbolic and their identity as "gamers" was reconstituted in the imaginary as the protectors of games. This passage $\grave{a}$ l'acte re-entrenched these gamers more deeply in their beliefs and fuelled their (sometimes violent) revolt against the critical feminists scholars that studied their games. The delusions of Gamergate circle around the paranoid belief that feminists are working to censor the games that they love, and this paranoia has been extending more broadly to concerns that developers are colluding with leftist ideologues to fill games with political propaganda, which was illustrated in the earlier example of Wolfenstein II. As Carolyn Jong writes in her analysis of their movement,

GamerGaters decry feminists that they believe are interfering with the hallowed workings of the "free market," claiming that their own relatively narrow view of games and gaming culture is "objective," "natural," "rational," and free from "ideology." While this type of policing may help to preserve (for a while) particular forms of cultural capital for gamers, it ultimately serves the interests of industry elites, and those who profit from the sale of games, by alleviating the pressure for change. (10)

While attempting to protect games from the imagined censorship of feminists, their efforts impose an inverted self-censorship that restricts games to an even more limited form common among AAA titles that feature male protagonists, first-person shooter mechanics, and damsels to be protected and saved.

In her analysis of the challenges faced by women who work in (and play) games, Katherine Cross argues that what facilitates this harassment is the "[m]öbius-strip of reality and unreality in gaming culture - a two sided and yet one-sided system of norms. 
The culture becomes real when it is convenient, and unreal when it is not; real enough to hurt people in, unreal enough to justify doing so" (4-5). In Lacanian terms, those who participate in these aggressions are stuck in the imaginary: games and the internet are not "real," except when one can leverage these spaces to cause real harm by inciting fear through violent threats and acts. As a result of being stuck in the imaginary, a critical approach based on the process of resolving neurosis would not simply be ineffective against such a group, but would be likely to trigger a psychotic break.

The inverse of The Beginner's Guide, therefore, would be a less hermeneutic and more narrative-based form that provides, for example, a rationale for the state of the world. In contrast to the utopian promises of the analytic approach to neuroses, it is difficult to imagine a critical media theory that is based on Lacan's delusional metaphor. While this approach may not offer the same transformative power as the former, its reliance on narrative does resist the hold of communicative capitalism which would otherwise accelerate the symptoms of psychosis; as Fink notes, there exists a "common propensity among psychotics to write" (Fundamentals of Psychoanalytic Technique 271). As an example, one might look to the "Make America Great Again" rhetoric of the Republican party during the 2016 United States presidential election, which captivated those in need of a rationale for mass unemployment, poverty, and political instability.

In The Playstation Dreamworld, Bown describes how pastoral and dystopian narrative in games such as Stardew Valley and Fallout function problematically as representations that justify our current dependence on capitalism and inability to imagine other possibilities: 
both dystopia and utopia have been appropriated to make capitalism appear to be the 'only alternative' by naturalizing a timeline that runs from barbarity to capital. Such patterns aim at the unconscious ingraining of a kind of capitalist conception of history, producing an appearance of uninterruptible linearity from pastoral national serenity to dystopia wasteland. The chance to envisage changes to capitalist modernity is eradicated, leaving only dreams of tempering its destructiveness (Stardew Valley) or of starting afresh after the apocalypse (Fallout). (49)

While these games lack the desired critical lens, they embody a kind of model that could be leveraged in service of critical media theory through their explanatory power. Dean similarly addresses and critiques these dystopian narratives and games for sustaining the myth of the individual; as she writes,

The survivor is a compelling identity under conditions of extreme competition and inequality. It validates surviving by any means necessary. Survival is its own reward. Setbacks and lapses are new challenges, ultimately greater proof of one's survival skills. Popular culture provides a wide array of survivors to emulate (as well as examples of those who have been unable to get themselves together): from Katniss in The Hunger Games, to the winners of uncountable reality television competitions, to games like Day Z and Fallout, to victims of illness or crime. (Crowds and Party)

Yet, a new narrative that does not rely on the individual might reverse these concerns about the dystopian narratives; what might, for instance, the narrative of the crowd look 
like? That a delusional metaphor must not be hermeneutic does not imply that it needs to be conservative.

In addition to providing a meaning that holds the world together in place of a paternal metaphor, Fink argues that the delusional metaphor should also give the psychotic a purpose in this world (256). As an example of such a narrative, Osmotic Studios' Orwell series stands out. One would be correct to assume from its title that the games pay tribute to George Orwell's Nineteen Eighty-Four (1949). In both Keeping an Eye On You (2016) and Ignorance is Strength (2018), players work for "The Nation" as agents in a surveillance program. Using the Orwell surveillance system, players are tasked with uncovering information about persons of interest who are connected to suspected terrorists. By analyzing public blogs and social media posts, players gain access to more secure forms of data on cellphones and private computers. This information is used to prevent crime, but its acquisition comes at the cost of citizens' privacy and is also used to control public information about government activity. Through its narrative, the series imagines the controversial implications of governments making use of the data that is willingly and publicly shared online by individuals, and as a result, it fuels distrust for the networks that circulate our information. As opposed to the self-analytical approach of The Beginner's Guide, Orwell creates a rationale for our exit from communicative capitalism. This approach may lack the kind of critical training to which Wreden's game aspires, but it nevertheless offers a challenge to communicative capitalism, even if players arrive at this conclusion in very different ways.

Because of the centrality of narrative to this psychoanalytic approach to critical media theory, one might be quick to conclude that games are not an essential component 
of this work. For Dean, books are inseparable from her critical media theory. Yet, as this study has argued, games offer similar (and new) modes of intervening and disrupting the circuits of drive that fuel communicative capitalism. Bogost and other ludologists reject the value of narrative for games, as has become apparent over the years, however, the repressed always returns. Stories may not be the horizon that ludologists want for games, but they have much to offer critical media studies that pure simulation cannot. This claim reveals why the suspension of narrative in communicative capitalism is central to its perpetuation.

There remain, however, a number of obstacles that stand in the way of the success of a critical media theory of games: the labour issues that Nick Dyer-Witheford addresses in his work on cognitive capitalism in the game industry; the economic and creative challenges identified by Alison Harvey that stunt the development of risky and experimental projects; and the vocal communities that oppose such changes due to paranoid conspiracies that developers are attempting to capitalize on liberal and leftist agendas. Overcoming these obstacles requires a reimagining of our current economic and political systems. Dean's work in Crowds and Party offers a meaningful step toward taking action by mobilizing the crowd through the party, but the kinds of critical games described herein may be instrumental to inspiring the emergence of the crowd. 


\section{Bibliography}

Aarseth, Espen. Cybertext: Perspectives on Ergodic Literature. Johns Hopkins UP, 1997.

Agamben, Giorgio. The Coming Community. Translated by Michael Hardt, U of Minnesota P, 1993.

Aikatsurama. "Lacanian Psychoanalysis and Ego Psychology: Two Sides of the Same Coin.” Psychoanalysis and Speculative Realism. 11 Sept. 2009.

Aldred, Jessica and Brian Greenspan, “A Man Chooses, A Slave Obeys: Bioshock and the Dystopian Logic of Convergence," Games and Culture vol. 6, no. 5, 2011, pp. 479-96.

Althusser, Louis. On the Reproduction of Capitalism: Ideology and Ideological State Apparatuses. 1971. Translated by G. M. Goshgarian, Verso, 2014.

Altman, Rick. A Theory of Narrative. Columbia UP, 2008.

Anthropy, Anna. "Love, Twine, and the End of the World." State of Play: Creators and Critics on Video Game Culture, Seven Stories Press, 2015, pp. 31-53.

Aristotle. "Poetics." Narrative Theory: Major Issues in Narrative Theory. Edited by Mieke Bal, Routledge, 2004, pp. 59-64.

Atari: Game Over. Directed by Zak Penn, Fuel Entertainment USA, 2014.

Avent, Ryan. "Escape to Another World." The Economist: 1843, Apr./May 2017.

Babe. Directed by Chris Noonan, performances by Christine Cavanaugh, James Cromwell, Magda Szubanski, and Hugo Weaving, Universal Pictures, 1995.

Bal, Mieke. Narratology: Introduction to the Theory of Narrative. 3rd ed., U of Toronto P, 2009.

Barthes, Roland. Image-Music-Text. Translated by Stephen Heath, Hill and Wang, 1977. 
Bastion. Supergiant Games, 2011. Video Game.

Batti, Bianca and Alisha Karabinus. "A Dream of Embodied Experience: On Ian Bogost, Epistemological Gatekeeping, and the Holodeck.” NYMGamer.com, 1 May 2017.

The Beginner's Guide. Everything Unlimited, Ltd., 2015. Video Game.

BioShock. 2K Boston, 2K Australia, 2007. Video Game.

BioShock Infinite. Irrational Games, 2013. Video Game.

Bissell, Tom. "Does the Sneaker Have to Matter? An Interview with Ken Levine, the Mind Behind BioShock." Grantland, 26 Mar. 2013.

Bissell, Tom et al. "When Story Is the Gameplay: Multi-Genre Writing for Telltale Games.” Game Developers Conference, San Francisco, CA, GDC Vault, 3 Mar. 2015.

Bogost, Ian. Persuasive Games: the Expressive Power of Videogames. MIT Press, 2007.

—. "Video Games Are Better Without Characters." The Atlantic, 13 Mar. 2015.

—. "Video Games Are Better Without Stories." The Atlantic, 25 Apr. 2017.

Bolter, Jay David and Richard Grusin. Remediation: Understanding New Media, MIT Press, 1999.

Borges, Jorge Luis. "The Aleph.” Aleph and Other Stories, translated by Andrew Hurley, Penguin Books, 2004, pp. 118-33.

Bowlingotter. “The Beginner's Guide.” YouTube, uploaded by Bowlingotter, 10 Oct. 2015, youtu.be/JVFDz018kr0.

Bown, Alfie. Enjoying It: Candy Crush and Capitalism. Zero Books, 2015.

—. "How Video Games Are Fuelling the Rise of the Far Right." The Guardian, 12 Mar. 2018. 
—. The Playstation Dreamworld. Polity, 2018.

Braid. Number None, 2009. Video Game.

@ BRKeogh. "i got way too much to do today so here is one word-vomit take on games and stories and I'm out now.” Twitter, 25 Apr. 2017, 6:25 p.m. Image.

Burroughs, William S. Naked Lunch. 1959. Grove Press, 2013.

Calleja, Gordon. In-Game: From Immersion to Incorporation. Cambridge. MIT Press, 2011.

Campbell, Colin. "Baldur's Gate Studio Responds to Harassment Over Trans Character." Polygon, 6 Apr. 2016.

Candy Crush Saga. King, 2012. Video Game.

Chaouli, Michel. "How Interactive Can Fiction Be?” Critical Inquiry, vol. 31, no. 3, 2005, pp. 599-617.

Chatman, Seymour Benjamin. Story and Discourse: Narrative Structure in Fiction and Film. Cornell UP, 1978.

Consalvo, Mia. “There Is No Magic Circle.” Games and Culture, vol. 4, no. 4, 2009, pp. 408-17.

Coulson, Mark and Christopher Ferguson. "The Influence of Digital Games on Aggression and Violent Crime." The Video Game Debate: Unravelling the Physical, Social, and Psychological Effects of Digital Games, edited by Rachel Kowert and Thorsten Quandt, Routledge, 2016, pp. 54-73.

Cuphead. StudioMDHR Entertainment Inc., 2017. Video Game.

Dahlen, Chris. Interview with Jonathan Blow. "Game designer Jonathan Blow: What We All Missed about Braid.” AV Club, 27 Aug., 2008. 
darklarke. “Mass Effect 3 Extended Cut.” BioWare, 5 Apr. 2012.

Dean, Jodi. Blog Theory:Feedback and Capture in the Circuits of Drive. Polity, 2010.

—. "Communicative Capitalism: Circulation and the Foreclosure of Politics." Cultural Politics, vol. 1, no.1, 2005, pp. 51-74.

-. Crowds and Party. Verso, 2016. Ebook.

—. "Not Us, Me." Verso Blog, versobooks.com, 26 Nov. 2017.

Deleuze, Gilles. "What is the Creative Act?" Two Regimes of Madness, Texts and Interviews 1975-1995. Translated by Ames Hodges and Mike Taormina, MIT Press, 2007, pp. 312-24.

Depression Quest. The Quinnspiracy, 2014.

Dini, Kourosh. "On Video Games, Culture, and Therapy.” Psychoanalytic Inquiry, vol. 32, no. 5, 2012, pp. 495-505.

Dragon's Lair. Digital Leisure Inc., 1983. Video Game.

Drone. Directed by Tonje Hessen Schei, Volt Film, 2014.

Dyer-Witheford, Nick. "Cycles and Circuits of Struggle in High-Technology Capitalism." Cutting Edge: Technology, Information Capitalism and Social Revolution, edited by Jim Davis et al., Verso, 1997, pp. 195-242.

Dyer-Witheford, Nick and Greig de Peuter. Games of Empire: Global Capitalism and Video Games. U of Minnesota P, 2009.

Dymek, Mikolaj. "Video Games: A Subcultural Industry." The Video Game Industry: Formation Present State, and Future, edited by Peter Zackariasson and Timothy L. Wilson, Routledge, 2012, pp. 34-56.

Empire. Directed by Andy Warhol, Warhol Films, 1964. 
Eskelinen, Markku. “The Gaming Situation.” Game Studies, vol. 1, no. 1, 2001, n.pag. E.T. the Extra-Terrestrial. Atari, 1982. Video Game.

Evans, Dylan. An Introductory Dictionary of Lacanian Psychoanalysis. Routledge, 1996.

Everything Unlimited Ltd. “The Beginner's Guide Trailer.” Online video clip. Steam. 1 Oct. 2015.

Fable. Big Blue Box, 2004. Video Game.

Fink, Bruce. A Clinical Introduction to Lacanian Psychoanalysis: Theory and Technique. Harvard UP, 1997.

—. Fundamentals of Psychoanalytic Technique: A Lacanian Approach for Practitioners. W. W. Norton \& Company, 2007.

—. The Lacanian Subject: Between Language and Jouissance. Princeton UP, 1995.

Fisher, Mark. Capitalist Realism: Is T here No Alternative? Zero Books, 2009.

Flanagan, Mary. Critical Play: Radical Game Design. MIT Press, 2009.

Fludernik, Monika. An Introduction to Narratology, translated by Patricia HäuslerGreenfield, Routledge, 2009.

Follows, Stephen. "How many films are released each year?” Stephen Follows Film Data and Education stephenfollows.com. 14 Aug. 2017.

Football Manager Mobile. Sports Interactive, 2006. Video Game.

"Four Walls and a Roof." The Walking Dead, created by Robert Kirkman, season 5, episode 3, American Movie Classics, 2014.

Frank, Allegra. "Steam's Biggest Seller of Shovelware Pulls Out of Game Business.” Polygon. 2 Oct., 2017. 
Frasca, Gonzalo. "Ludologists Love Stories, Too: Notes from a Debate that Never Took Place." Ludology.org, 2003.

—. "Simulation versus Narrative: Introduction to Ludology." The Video Game Theory Reader, edited by Mark J. P. Wolf and Bernard Perron, Routledge, 2003, pp. 22136.

—. "Videogames of the Oppressed: Critical Thinking, Education, Tolerance, and Other Trivial Issues." First Person: New Media as Story, Performance, and Game, edited by Noah Wardrip-Fruin and Pat Harrigan, MIT Press, 2004, pp. 85-94.

—. Videogames of the Oppressed: Videogames as a means for Critical Thinking and Debate. Dissertation, Georgia Institute of Technology, 2001.

Freud, Sigmund. Beyond the Pleasure Principle, translated by James Strachey. W. W. Norton \& Co., 1975.

—. "Creative Writers and Day-Dreaming." The Freud Reader. Translated by James Strachey, edited by Peter Gay, W.W. Norton \& Co., 1989, pp. 436-43.

—. "The Subtleties of a Faulty Action.” Freud Complete Works. 4817-21. PDF.

—. "The Unconscious." The Freud Reader, edited by Peter Gay, W.W. Norton \& Co., 1989, pp. 572-83.

Fullerton, Tracey. Game Design Workshop: A Playcentric Approach to Creating Innovative Games. Morgan Kaufmann Publishers, 2008.

Galloway, Alexander R. Gaming: Essays on Algorithmic Culture. University of Minnesota Press, 2006.

-. The Interface Effect. Polity, 2012.

Galyonkin, Sergey. “The Indie Games Are Too Damn Cheap.” Galyonk.in 13 Aug. 2017. 
Garland, Ian. “Fan, 21 Lands Top Manager's Job After Wowing on Football Manager Game.” The Sun, 23 Nov. 2012.

Genette, Gérard. Narrative Discourse Revisited. Translated by Jane E. Lewin, Cornell UP, 1988.

Gerrig, Richard. Experiencing Narrative Worlds: On the Psychological Activities of Reading. Yale UP, 1993.

Gone Home. The Fullbright Company, 2013. Video Game.

Good, Owen S. "No Man's Sky Creator: I've Gotten Death Threats Over Delay News." Polygon, 29 May 2016.

Grand Theft Auto. Rockstar Games, 1997-2013. Video Game.

Green, Melanie C. "Transportation Into Narrative Worlds: The Role of Prior Knowledge and Perceived Realism." Discourse Processes, vol. 38, no. 2, 2004, pp. 247-66.

Grubb, Jeff. “Why Triple-A Devs Are Going Indie (and Why Indies Aren’t Going TripleA)." Venture Beat, 7 Sept., 2017.

Grusin, Richard. "Premediation.” Criticism, vol. 46, no. 1, 2004, pp. 17-39.

Harvey, Alison. “Twine's Revolution: Democratization, Depoliticization, and the Queering of Game Design.” The Italian Journal of Game Studies, vol. 1, no. 3, 2014, pp. 95-107.

Hayles, N. Katharine. Electronic Literature: New Horizons for the Literary. Notre Dame, IN: University of Notre Dame, 2008.

Hester, Blake. “'PUBG' Breaks Its Concurrent Record, Has Banned 1.5 Million Players.” The Rolling Stone, 29 Dec. 2017.

Home. Benjamin Rivers Inc, 2012. Video Game. 
Hudson, Kent. “The System Is the Message: Dynamic Narrative in The Novelist." Game Developers Conference, San Francisco, CA, GDC Vault, 19 Mar. 2014.

Huizinga, Johan. Homo Ludens: a Study of the Play-Element in Culture. 1944. Routledge, 1980.

Hunicke, R., et al. "MDA: A Formal Approach to Game Design and Game Research." Northwestern University, no date, pp.1-5.

Hutcheon, Linda. A Theory of Adaptation. Routledge, 2006.

@ (ibogost. "Both extremes of gaming are pissed at me - the mainstream traditionalists \& the fringe progressivists. And neither can see or hear the other." Twitter, 26 Apr. 2017, 8:58 p.m.

Irwin, Mary Jane. “Cooking Up A Blockbuster Game.” Forbes.com, 21 Nov. 2008.

Isbister, Katherine. How Games Move Us: Emotion by Design, MIT Press, 2016.

It's No Game. Directed by Oscar Sharp, performances by David Hasselhoff, End Cue, 2017.

Jackson, Robert. BioShock: Decision, Forced Choice and Propaganda. Zero Books, 2014

jagodzinski, jan. "Video gGame Cybersubjects, the Ethics of Violence and Addiction: A Psychoanalytic Approach.” Psychoanalysis, Culture \& Society, vol. 11, no. 3, 2006, pp. 282-303.

Jameson, Fredric. Postmodernism, or, the Cultural Logic of Late Capitalism. Duke UP, 1991.

Jenkins, Henry. Convergence Culture: Where Old and New Media Collide. New York UP, 2006. 
Jong, Carolyn. “'Fighting the Good Fight:' GamerGate, NotYourShield, and NeoFascism.” Concordia University, 2014, pp. 1-16. PDF.

Juul, Jesper. The Art of Failure: An Essay on the Pain of Playing Video Games. MIT Press, 2013.

—. "Games Telling Stories? A Brief Note on Games and Narratives." Game Studies, vol. 1, no. 1,2001 , n. pag.

—. "Without a Goal." Videogame/Player/Text, edited by Tanya Krzywinska and Barry Atkins, Manchester UP, 2007, pp. 191-206.

Katamari Damacy. Namco, 2004. Video Game.

Kirkman, Robert, Charlie Adlard, and Cliff Rathburn. "Fear the Hunters." The Walking Dead, vol. 11, no. 64, 2009.

Lacan, Jacques. The Seminar of Jacques Lacan: Book 2: The Ego in Freud's Theory and in the Technique of Psychoanalysis 1954-1955. Translated by Sylvana Tomaselli. Edited by Jacques-Alain Miller, Cambridge UP, 1988.

—. The Seminar of Jacques Lacan: Book 7: The Ethics of Psychoanalysis 1959-1960. Translated by Dennis Porter. Edited by Jacques-Alain Miller, Cambridge UP, 1986.

Landow, George P. Hypertext 3.0: Critical Theory and New Media in an Era of Globalization. Johns Hopkins UP, 2006.

Lange, Amanda. “'You're Just Gonna Be Nice': How Players Engage with Moral Choice Systems." Journal of Games Criticism, vol. 1, no. 1, 2014, pp. 1-16.

Late Shift. CtrlMovie, Wales Interactive, 2017. Video Game.

Lear, Johnathan. Open Minded:Working Out the Logic of the Soul. Harvard UP, 1998. 
Let's Play: Ancient Greek Punishment. Pippin Barr, 2012. Video Game.

Levine, Ken. "Narrative Legos: Building Replayable Narrative Out of Lots of Tiny Pieces." Game Developers Conference, San Francisco, CA, GDC Vault, 21 Mar. 2014.

Lizardi, Ryan. "BioShock: Complex and Alternate Histories." Game Studies, vol. 14, no. 1, 2014, n.pag.

Lo, Claudia. “How RimWorld's Code Defines Strict Gender Roles.” Rock Paper Scissors, 2 Nov. 2016.

The Magic Circle. Question Games, 2015. Video Game.

Manovich, Lev. The Language of New Media. MIT Press, 2002.

Marcin, Tim. 'Nazi-Killing Video Game 'Wolfenstein II' Angers Nazis with 'Make America Nazi-Free Again’ Slogan.” Newsweek, 6 Oct. 2017.

Mateos-Garcia, Juan, et al. "Sticking to Their Guns: the Impact of the Culture and Organisational Practices of Video Games Studios on the Technological Trajectory of the Console Games Sector." Opening Up Innovation: Strategy, Organization and Technology. Presented at the DRUID Summer Conference, London, UK, June 2010.

Mattas, Jeff. Interview with Davey Wreden. "Interview: The Stanley Parable Developer Davey Wreden." Shack News, 27 Sept. 2011.

McGonigal, Jane. “Gaming Can Make a better World.” YouTube, uploaded by TED, 17 Mar. 2010, youtu.be/dE1DuBesGYM.

-. Reality is Broken: Why Games Make Us Better and How They Can Change the World. Penguin, 2011. 
McGowan, Todd. The End of Dissatisfaction: Jacques Lacan and the Emerging Society of Enjoyment. State U of New York P, 2012.

Morris, David Z.. "Young American Men Are Choosing Video Games Over Work in Staggering Numbers.” Fortune, 16 July 2017.

Morrison, Brice. "Meaningful Choice in Games: Practical Guide \& Case Studies." Gamasutra, 11 Nov. 2013.

Mountain, David OReilly, 2014. Video Game.

Mukherjee, Souvik. Video Games and Storytelling: Reading Games and Playing Books. Palgrave Macmillan, 2015.

Murray, Janet. Hamlet on the Holodeck: The Future of Narrative in Cyberspace. MIT Press, 1998.

—."The Last Word on Ludology v Narratology." inventingthemedium.com, 28 June 2013.

Never Alone (Kisima Ingitchuna). Upper One Games, E-Line Media, 2014. Video Game. No Man's Sky. Hello Games, 2016. Video Game.

The Novelist. Orthogonal Games, 2013. Video Game.

Ore, Jonathan. “The Video Game Cuphead Is a Made-In-Canada Tribute to Animation's Golden Age: Chad and Jared Moldenhauer Quit Their Jobs, Remortgaged Their Homes — and Made an Instant Classic.” CBC News, 15 Oct. 2017.

Orwell, George. Nineteen Eighty-Four. 1949. Harper Perennial, 2014. Orwell: Ignorance is Strength. Osmotic Studios, 2018. Video Game. Orwell: Keeping an Eye On You. Osmotic Studios, 2016. Video Game. 
Page, Ruth and Bronwen Thomas. "Introduction." New Narratives. Eds. Ruth Page and Bronwen Thomas. U of Nebraska P, 2011, pp. 1-19.

Papale, Luca. "Beyond Identification: Defining the Relationships between Player and Avatar.” Journal of Games Criticism, vol. 1, no. 2, 2014, pp 1-12.

Papers, Please. Lucas Pope, 2013. Video Game.

Parker, Ian. Psychoanalytic Culture: Psychoanalytic Discourse in Western Society. Sage Publications, 1997.

Pettman, Dominic. Infinite Distraction. Polity, 2016.

Pfaller, Robert. Interpassivity: The Aesthetics of Delegated Enjoyment. Edinburgh UP, 2017.

—. "Little Gestures of Disappearance: Interpassivity and the Theory of Ritual." Journal of European Psychoanalysis, no. 16, 2003.

PlayerUnknown's Battlegrounds. PUBG Corporation, 2017. Video Game.

poochyena. "Not Recommended.” A Review of The Beginner's Guide. Steam, 2 Oct. 2015.

Prince, Gerald. "The Disnarrated.” Narrative Theory: Major Issues in Narrative Theory. Edited by Mieke Bal. Routledge, 2004, pp. 297-305.

Proteus. Ed Key and David Kanaga, 2013. Video Game

Quinn, Zoë. Crash Override: How Gamergate (Nearly) Destroyed my Life, and How We Can Win the Fight Against Online Hate. Public Affairs, 2017.

Raley, Rita. "TXTual Practice." Comparative Textual Media: Transforming the Humanities in the Postprint Era, edited by N. Katherine Hayles and Jessica Pressman. U of Minnesota P, 2013, pp. 5-32. 
Rehak, Bob. "Playing at Being: Psychoanalysis and the Avatar." The Video Game Theory Reader, edited by Mark J. P. Wolf and Bernard Perron, Routledge, 2003.

Ricœur, Paul. "Life in Quest of Narrative." On Paul Ricoeur: Narrative and Interpretation, edited by David Wood, Routledge, 2002, pp. 20-33.

RimWorld. Ludeon Studios, 2016. Video Game.

“RimWorld." Steam, 15 July 2016.

Rivers, Benjamin. “About.” homehorror.com, 2012.

Robertson, Adi. "Watching Internet Nazis Get Mad at Wolfenstein II is Sadder than the Game's Actual Dystopia.” The Verge, 12 June 2017.

Ryan, Marie-Laure. "Allegories of Immersion: Virtual Narration in Postmodern Fiction." Style, vol. 29, no. 2, 1995, pp. 262-86.

—. Avatars of Story. University of Minnesota Press, 2006.

—. "The Interactive Onion: Layers of User Participation in Digital Narrative Texts." New Narratives, edited by Ruth Page and Thomas Bronwen, U of Nebraska P, 2011. $35-62$.

-. Narrative as Virtual Reality 2: Revisiting Immersion and Interactivity in Literature and Electronic Media. Johns Hopkins UP, 2015.

-.Possible Worlds, Artificial Intelligence, and Narrative Theory. $\mathrm{U}$ of Bloomington and Indianapolis P, 1991.

—. "Toward a Definition of Narrative." The Cambridge Companion to Narrative, edited by David Herman, Cambridge UP, 2007, pp. 22-35.

Salen, Katie and Eric Zimmerman. Rules of Play, MIT Press, 2004.

Salmon, Christian. Storytelling: Bewitching the Modern Mind. Verso, 2017. 
Scanlon, Michael et al. "The First Door: Gender, Authority and Choice in The Stanley Parable.” DiGRA, July 2017, Melbourne, VI, AU. DiGRA2017.com.

Shane, Anna. “'Babe” and the End of Analysis.” Lacan.org. 1 Feb. 1997.

Shaw, Adrienne. Gaming at the Edge: Sexuality and Gender at the Margins of Gamer Culture, University of Minnesota Press, 2014

Sicart, Miguel. “Against Procedurality.” Game Studies, vol. 11, no. 3, 2011: n. pag.

—. Beyond Choices: The Design of Ethical Gameplay, MIT Press, 2013.

Siegel, Marjorie. "More than Words: The Generative Power of Transmediation for Learning." Canadian Journal of Education, vol. 20, no. 4, 1995, pp. 455-75.

Simon, Bart. "Indie Eh? Some kind of Game Studies." Loading..., vol. 7, no. 11, 2013, pp. 1-7.

Simons, Jan. "Narrative, Games, and Theory." Game Studies, vol. 7, no. 1, 2007, n. pag. The Sims. Maxis, 2000. Video Game.

Sinclair, Brendan. Interview with Ken Levine. "Q\&A: Diving deeper into BioShocks Story." Gamespot, 20 Sept. 20, 2007.

Smethurst, Toby and Stef Craps. "Playing with Trauma: Interreactivity, Empathy, and Complicity in The Walking Dead Video Game." Games and Culture, vol. 10, no. 3, 2015, pp. 269-90.

Space Invaders. Taito, 1977. Video Game.

The Stanley Parable. Galactic Cafe, 2013. Video Game.

Star Wars. Atari, 1983. Video Game.

@SSteam_Spy. “38\% of all Steam games were released in 2016” Image. Twitter, 30 Nov. 2016, 4:19 p.m. Image. 
—. "\# of new games on Steam over years." Twitter, 14 Sept. 2017, 12:49 p.m. Image.

—. "7,672 games were released on Steam in 2017, 21 games per day on average. I will write my usual analysis a bit later this year, still dealing with the relocation and stuff..." Twitter, 10 Jan. 2018, 9:41 a.m.

Stranger, Matt. "How an Obsession with Football Manager Could Earn You a Career in the Game." The Guardian, 29 Sept. 2016.

Suits, Bernard. The Grasshopper: Games, Life, and Utopia. Broadview Press, 2005.

"Sunspring: A Sci-Fi Short Film Starring Thomas Middleditch." YouTube, uploaded by Ars Technica Videos, 9 June 2016, youtu.be/LY7x2Ihqjmc.

Sunspring. Directed by Oscar Sharp, performances by Thomas Middleditch, End Cue, 2016.

Superannuation. "How Much Does It Cost To Make A Big Video Game?" Kotaku, 15 Jan. 2014.

SuperBetter. Jane McGonigal. SuperBetter.com, 2012. Video Game.

SwingPoynt. "You NEED to see this game - The Beginner's Guide." YouTube. YouTube, 2 Oct. 2015, youtu.be/gT0_x3fSXdI.

Tamás, G. M. “On Post-Fascism: The Degradation of Universal Citizenship.” Boston Review, 1 June 2000.

Tetris. Pazhitnov, 1985. Video Game.

Thomas, Jordan. “What Do We Mean When We Say 'Indiepocalypse'?” Game Developers Conference, San Francisco, CA, GDC Vault, 15 Mar. 2016.

Thorne, Sarah. "Perverse and Interpassive Gaming: Enjoyment and Play in Gamespaces." Psychoanalysis, Culture \& Society, vol. 22, no. 1, 2017, pp. 106-13. 
To Be or Not to Be: That Is the Adventure. Tin Man Games, 2015. Video Game.

“Tropes vs. Women.” YouTube, uploaded by feministfrequency, 2011, youtu.be/uqJUxqkenKA.

Undertale. tobyfox, 2015. Video Game.

Walden, a Game. Tracy Fullerton, 2017. Video Game.

“The Walking Dead - Episode 1 Stats Trailer." Youtube, uploaded by Telltale Games, 27 June 2012, youtu.be/oS8JeNDdWs0.

“The Walking Dead - Episode 2 Stats Trailer." Youtube, uploaded by Telltale Games, 13 August 2012, youtu.be/akgcch6735Q.

“The Walking Dead." Steam. 24 Apr. 2012.

The Walking Dead. Telltale Games, 2012. Video Game.

Wark, McKenzie. Gamer Theory. Harvard UP, 2007.

What Remains of Edith Finch. Giant Sparrow, 2017. Video Game.

The Witcher. CD Projekt RED, 2007. Video Game.

The Witness. Thekla, Inc., 2016. Video Game.

@ wolfenstein. "There is only one side. \#NoMoreNazis \#Wolf2" Twitter, 15 Oct. 2017, 6:00 p.m. Video: twitter.com/wolfenstein/status/919684333207568385

Wolfenstein II: The New Colossus. MachineGames, 2017. Video Games.

World Without Oil. Jane McGonigal. WorldWithoutOil.org, 2007. Augmented Reality Game.

Wreden, Davey. “The Beginner's Guide - Let's Play Commentary.” Twitch, streamed by HelloCakebread, 28 Feb. 2016, twitch.tv/hellocakebread.

X-COM series. Firaxis Games, 1994-2016. Video Game. 
Zimmerman, Eric. "Gaming Literacy: Game Design as a Model for Literacy in the Twenty-First Century." The Video Game Theory Reader, edited by Bernard Perron and Mark J. P. Wolf. Routledge, 2009, pp. 23-31.

Žižek, Slavoj. “The Cyberspace Real.” The European Graduate School, 2009, accessed 9 Jan. 2015.

—. How to Read Lacan. Granta Books, 2006.

—. Looking Awry: And Introduction to Jacques Lacan through Popular Culture. MIT Press, 1992.

—. The Plague of Fantasies. Verso, 1997.

—. The Sublime Object of Ideology. Verso, 1989.

—. "Will You Laugh for Me, Please?” In These Times, 18 July 2003. 